\title{
Development of Resonating Oscillating Linear Engine Alternator; Instrumentation and Control
}

\author{
Fereshteh Mahmudzadeh
}

Follow this and additional works at: https://researchrepository.wvu.edu/etd

\author{
Recommended Citation \\ Mahmudzadeh, Fereshteh, "Development of Resonating Oscillating Linear Engine Alternator; \\ Instrumentation and Control" (2018). Graduate Theses, Dissertations, and Problem Reports. 7211. \\ https://researchrepository.wvu.edu/etd/7211
}

This Thesis is protected by copyright and/or related rights. It has been brought to you by the The Research Repository @ WVU with permission from the rights-holder(s). You are free to use this Thesis in any way that is permitted by the copyright and related rights legislation that applies to your use. For other uses you must obtain permission from the rights-holder(s) directly, unless additional rights are indicated by a Creative Commons license in the record and/ or on the work itself. This Thesis has been accepted for inclusion in WVU Graduate Theses, Dissertations, and Problem Reports collection by an authorized administrator of The Research Repository @ WVU. For more information, please contact researchrepository@mail.wvu.edu. 


\title{
Development of Resonating Oscillating Linear Engine Alternator; Instrumentation and Control
}

\author{
Fereshteh Mahmudzadeh
}

Thesis submitted to the

College of Engineering and Mineral Resources

at West Virginia University

in partial fulfillment of the requirements for the degree of

Master of Science

In

Electrical Engineering

Parviz Famouri, Ph.D., Chair

Muhammad A. Choudhry, Ph.D.

Katerina Goseva-Popstojanova, Ph.D.

Lane Department of Computer Science and Electrical Engineering

\author{
Morgantown, West Virginia
}

2018

Keywords: Linear Engine Alternator, Control

Copyright 2018 Fereshteh Mahmudzadeh 


\title{
Abstract \\ Development of Resonating Oscillating Linear Engine Alternator; Instrumentation and Control
}

\author{
Fereshteh Mahmudzadeh
}

The Oscillating Linear Engine and Alternator (OLEA) machine is a micro power generator that operates on natural gas. The OLEA is a permanent magnet linear tubular electric machine. It is driven by a two-stroke, free-piston internal combustion engine. The WVU team is developing the OLEA, which is a $1 \mathrm{KW}$ electricity generator with high efficiency, long life, compact size, and low cost for residential and commercial applications.

This research focuses on implementing and testing the instrumentation and control of a linear tubular permanent magnet alternator combined with a two-stroke, free-piston internal combustion engine into a single unit. A start-up mechanism has been implemented to start running the engine. Afterward, the controller regulates the engine performance by adjusting the combustion timings.

In order to start the engine and run the machine in generating mode, enough mechanical energy is required to be transferred to the system to provide minimal engine compression ratio. The alternator gate-driving signal is supposed to be synchronized to the translator movements to achieve the maximum transfer of energy and start the engine. Thus, a start-up mechanism is essential to acquire the preferred alignment of the driving voltage to the motion signal.

The OLEA machine is controlled by synchronizing the gate-driving signal with the translator position in the motoring mode. The control strategy during the machine start-up involves using a software-based Phase Locked Loop (PLL) system. A Linear Magnetic Hall Effect sensor is employed to collect the position data for the OLEA system. At the same time, the entire control procedure, including the motoring and generating modes, is designed according to real-time position information received by the position sensor.

Engine control in generating mode is accomplished by the ignition signal in real-time, according to the current position of the alternator. Similar to the startup, the engine ignition signal is generated by the PLL approach. The phase excitation voltage signals for startup and ignition 
signals for running are dependent upon the position signal, and they are synchronized with the machine movement.

A Digital Signal Processor (DSP) from Texas Instruments C2000 series has been utilized to run the algorithm for monitoring, diagnostics, and control tasks. Speed signal, which is used to generate the control signals, is obtained from the position data by the DSP. Moreover, an H_Bridge inverter is designed to drive the machine as a motor for a start-up.

Furthermore, data acquisition is performed through a Serial Peripheral Interface (SPI) communication protocol. The position, speed, and control signals are displayed and logged to the computer by the means of a Microchip MCP4921 Digital to Analog Converter (DAC) equipped with SPI interface.

The above techniques have been implemented and tested on the OLEA machine and the linear generator functioning is the result of this research. The details of the implementation are presented in this research and a technique is offered and tested to overcome the noise problems within the position detection system. 


\section{Table of Contents}

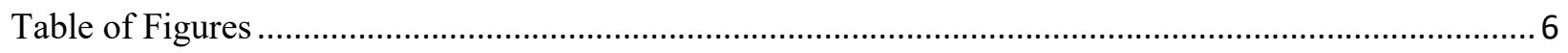

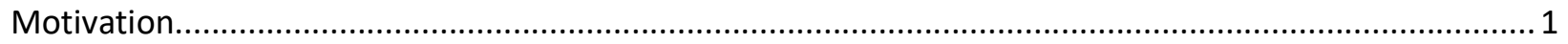

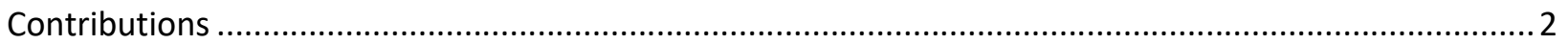

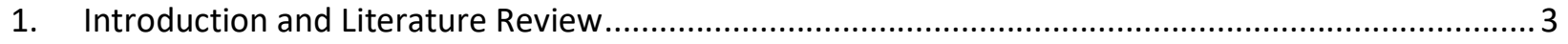

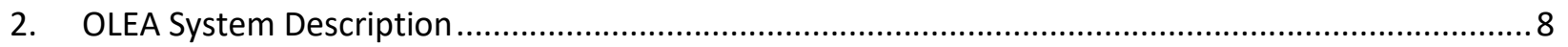

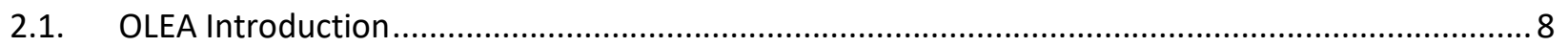

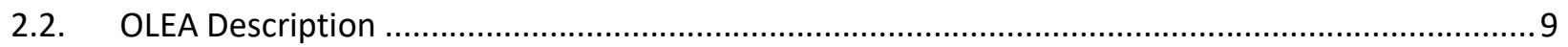

2.3. Linear Tubular Brushless Permanent Magnet Electric Machine .................................................. 10

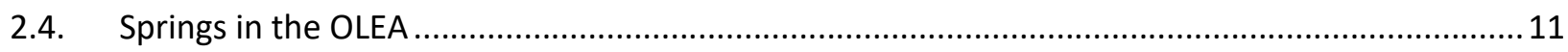

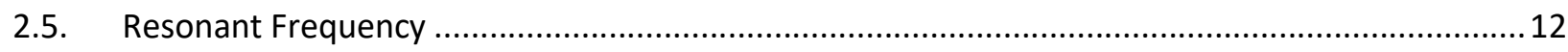

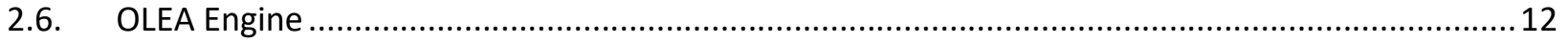

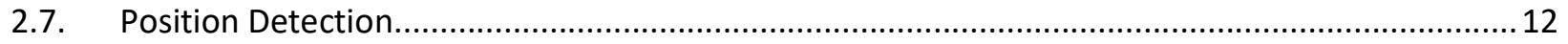

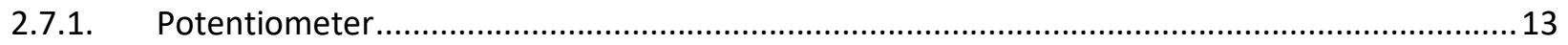

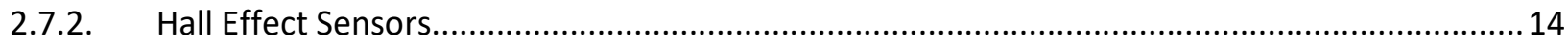

2.7.3. Position Detection by a Linear Incremental Magnetic Encoder ..............................................16

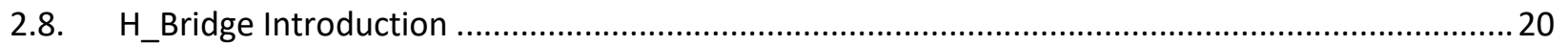

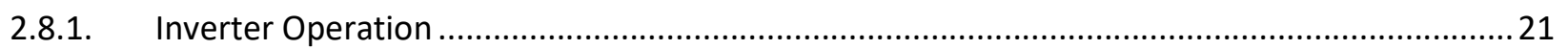

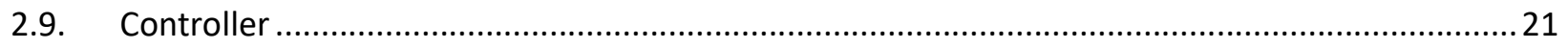

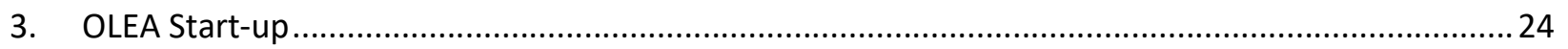

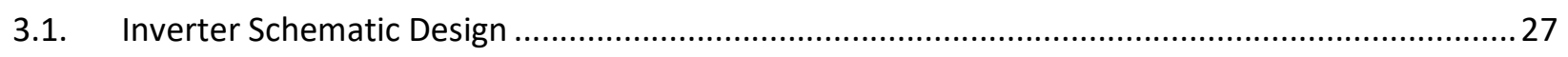

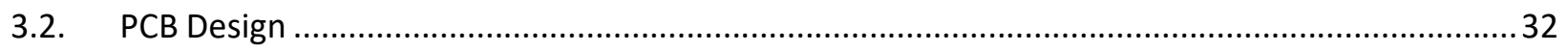

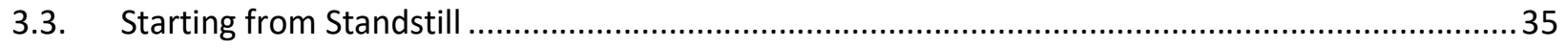

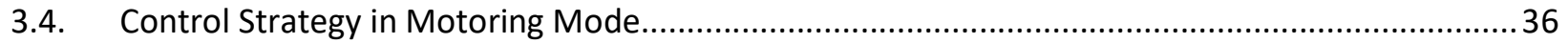

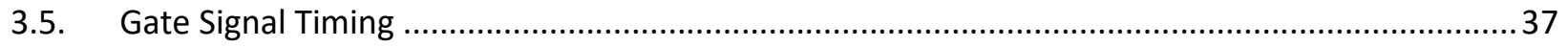

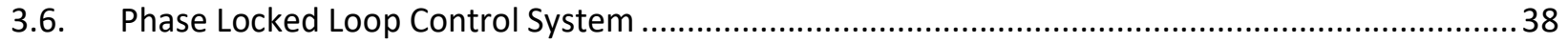

3.6.1. A Simple Description of the Mechanism of a Phase Locked Loop .............................................38

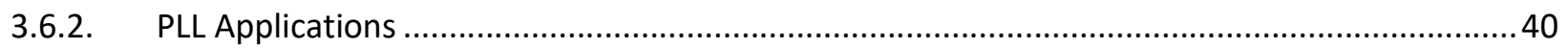

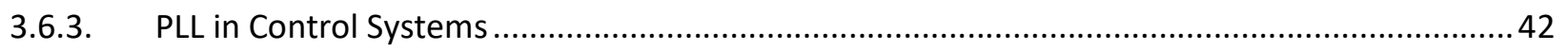

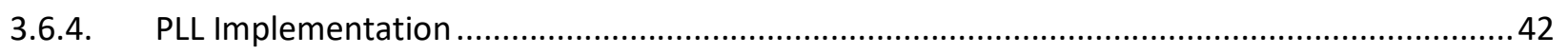

3.6.5. Secondary PLL Applications in Our System ..................................................................... 43

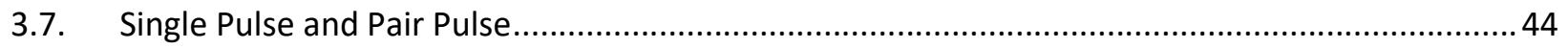

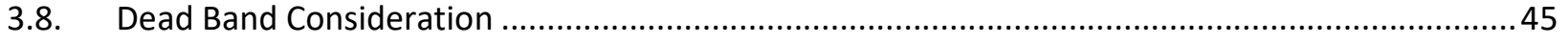




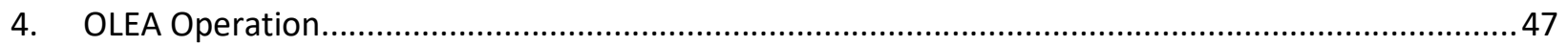

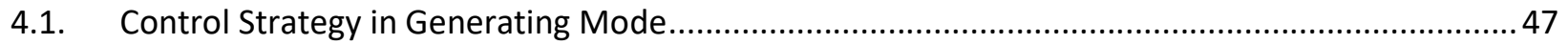

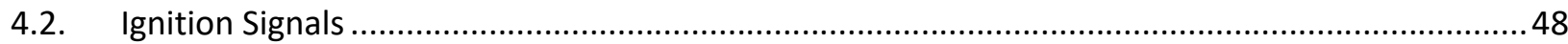

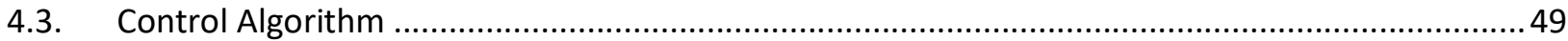

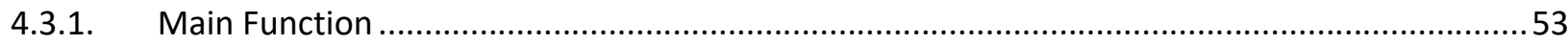

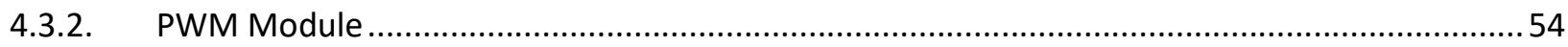

4.3.3. Timer Function - Speed Calculation and Signal Generation ....................................................55

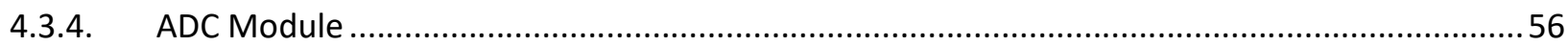

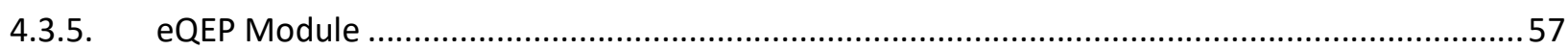

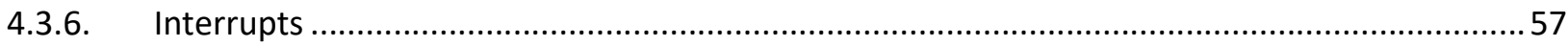

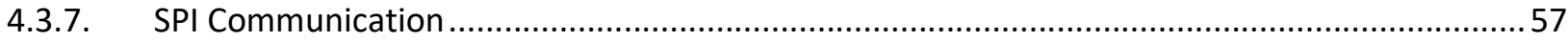

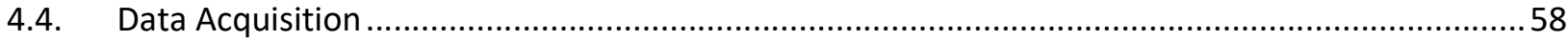

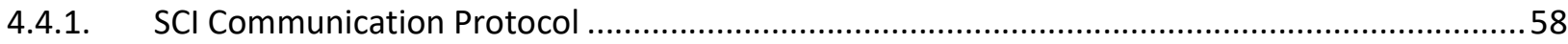

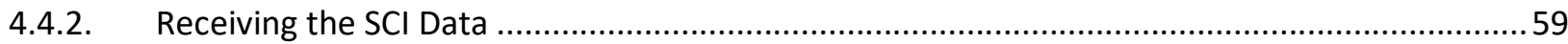

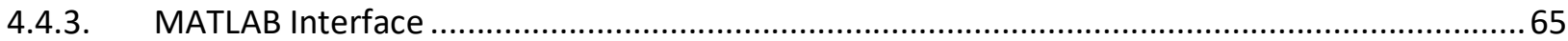

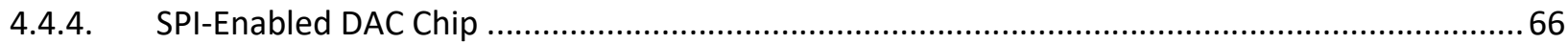

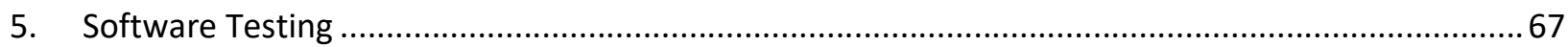

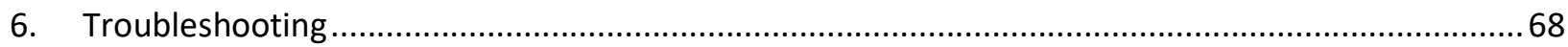

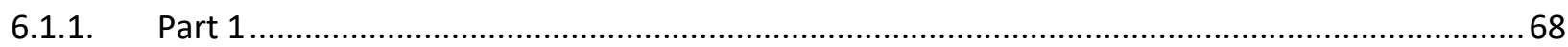

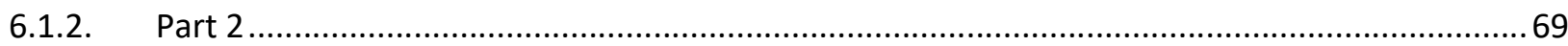

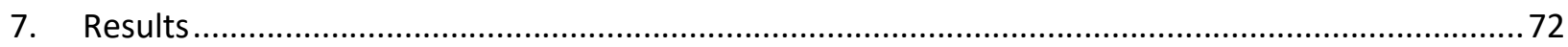

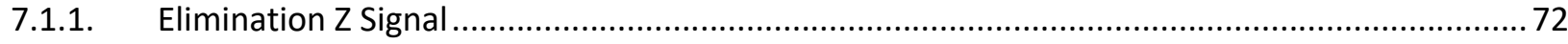

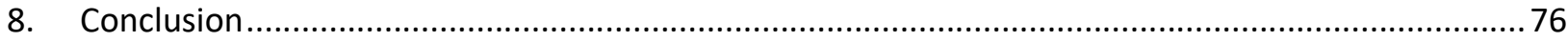

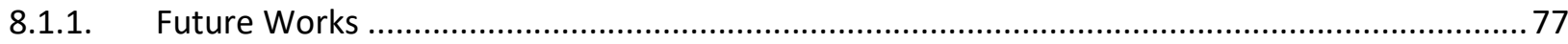

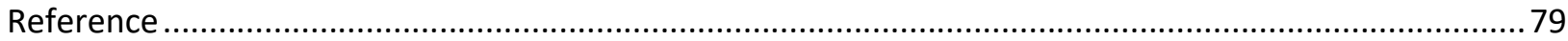




\section{Table of Figures}

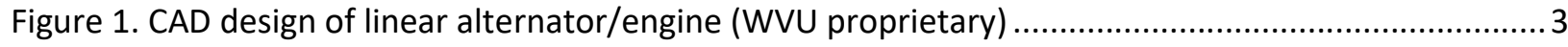

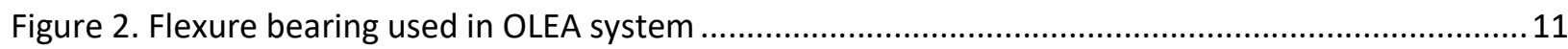

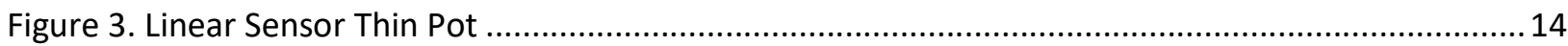

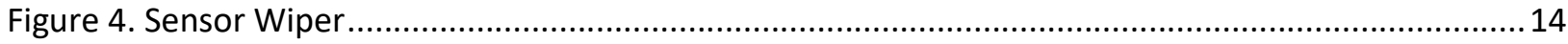

Figure 5. Timing Diagram - Incremental, Periodic Reference Mark .................................................... 17

Figure 6. Linear Incremental Magnetic Encoder.............................................................................. 17

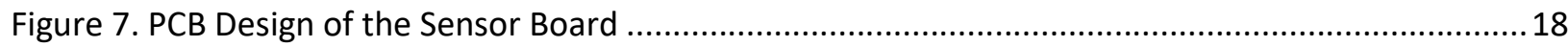

Figure 8. Position Sensor Mounted on the Printed Circuit Board ....................................................... 18

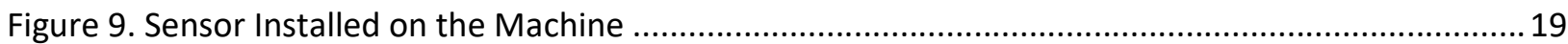

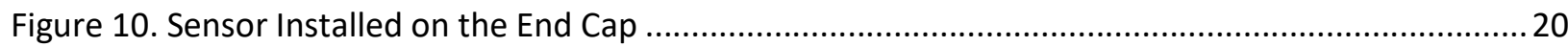

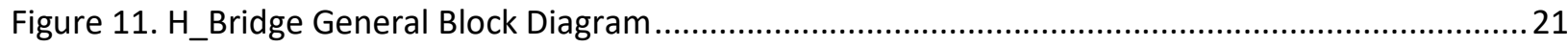

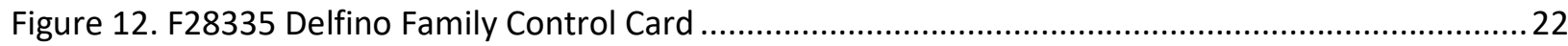

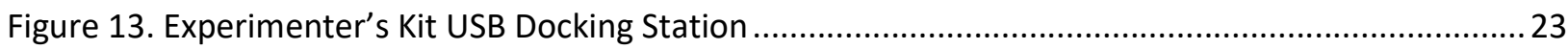

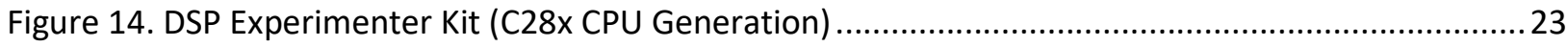

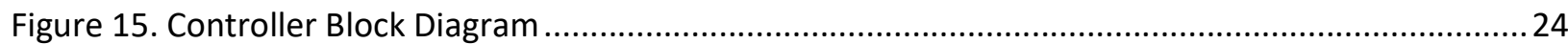

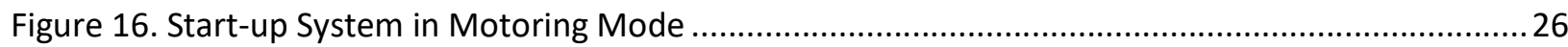

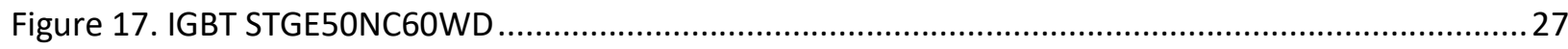

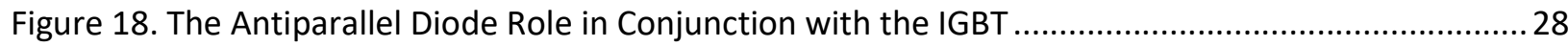

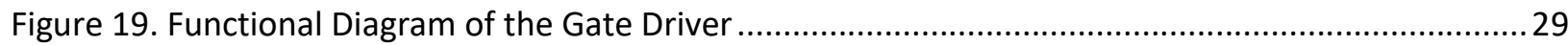

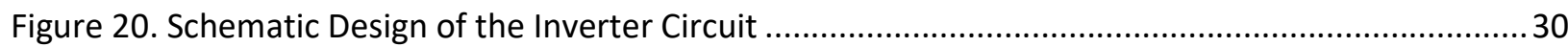

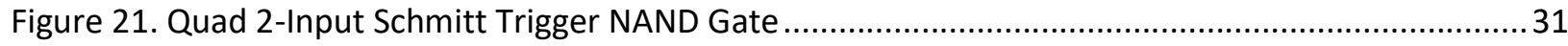

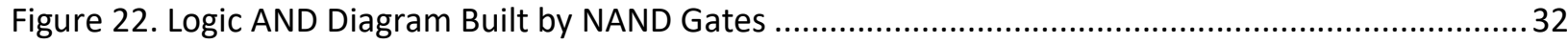

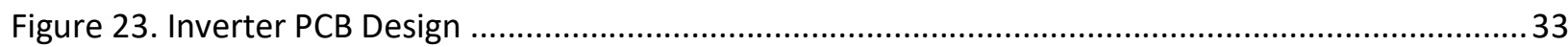

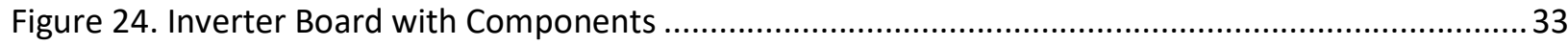

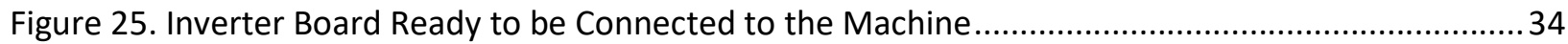

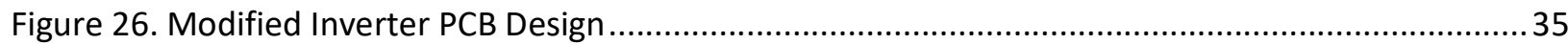

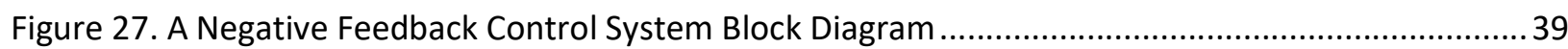

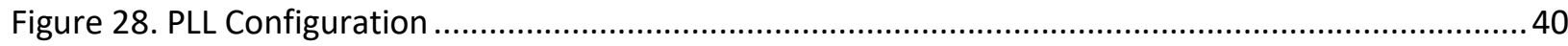

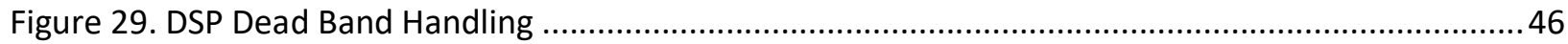

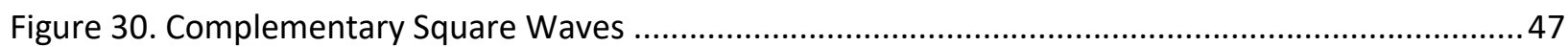

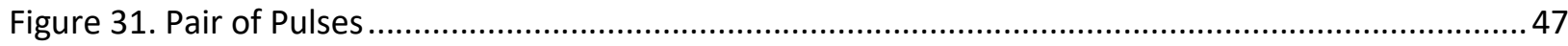

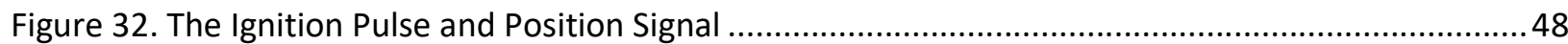

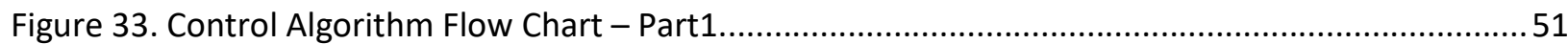

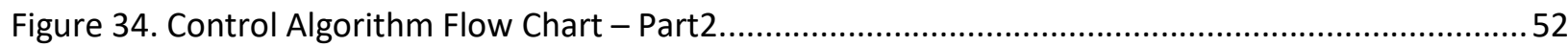

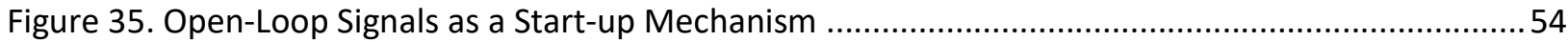

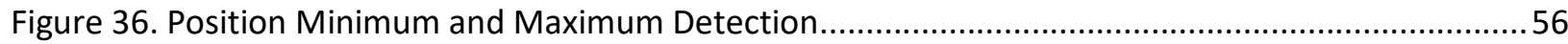

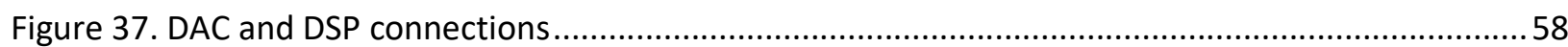

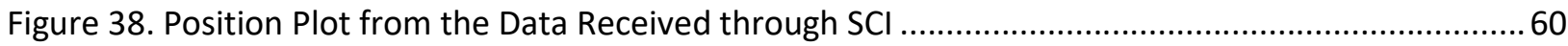

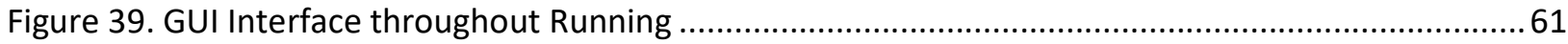

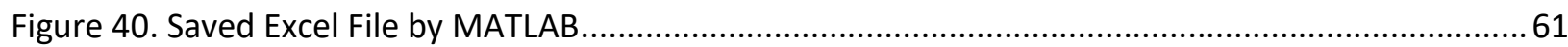

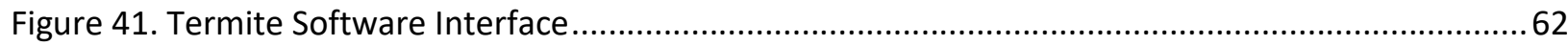

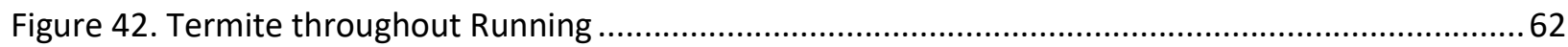

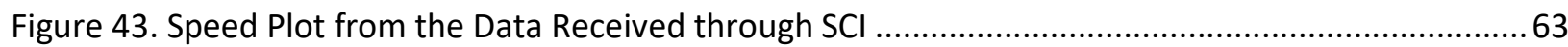


Figure 44. Position and PLL Pulses Plot from the Data Received through $\mathrm{SCl}$.....................................64

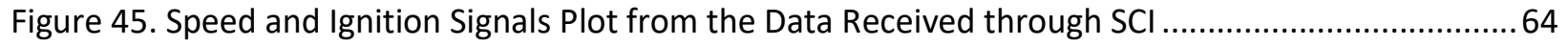

Figure 46. GUI Interface to Command the Open-Loop Run.................................................................65

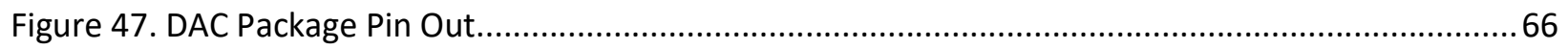

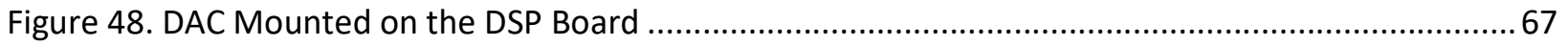

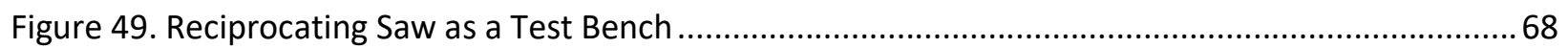

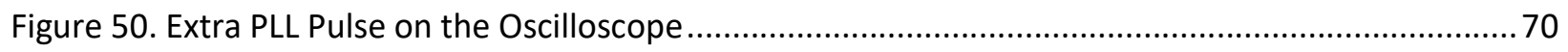

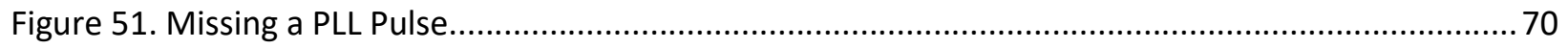

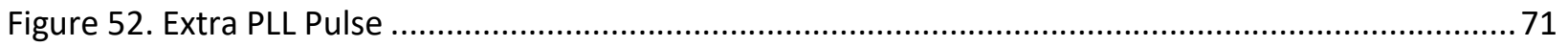

Figure 53. Unsmooth Position Signal but Handled .......................................................................... 71

Figure 54. The Position Signal from the Z Elimination Code on the Oscilloscope ...................................73

Figure 55. Position, PLL, and Ignition Signals from the Z Elimination Code on the Oscilloscope ............... 74

Figure 56. Position Signal, PLL Signal, and Ignition Signal Plot from the Z Elimination Code ..................... 74 


\section{Motivation}

The main motivation behind this thesis is developing a control mechanism for a linear machine, operating as a generator, to enhance the performance and reliability of the overall system.

A start-up mechanism is required to start running the engine and initiate electricity generation by receiving fuel. The start-up technique provides the minimum combustion ratio for the engine to start ignition. The minimum compression ratio cannot be supplied during the first stroke. Thus, the proposed start-up technique is offered to achieve the minimum stroke and compression ratio for the engine to start combustion.

Subsequently, to increase the efficiency and output power generation, the engine combustion timings need to be controlled. The lack of a control system to generate and adjust the ignition timings is another motivation, and fundamental to develop and implement the entire control procedure behind this thesis. 


\section{Contributions}

The main contributions of this thesis towards instrumentation and control of the resonating Oscillating Linear Engine Alternator are as follows:

$>$ Inverter design: A customized inverter has been designed and built to control the current direction through the machine phase windings during the motoring mode.

> DSP programming: A Digital Signal Processor has been utilized to perform the control tasks during the transient mode and the steady-state operation.

$\checkmark$ Starting and running the machine from a standstill state has been performed. The DSP is programmed to generate a pulse signal with a fixed frequency and fixed duty cycle in an open loop mode to create the reciprocating motion.

$\checkmark$ Start-up control is to supply sufficient stroke for the engine to start combustion. This function has been performed by the means of the DSP and a software-based PLL technique implementation. The DSP receives the position information from the position sensor and generates driving voltage pulses according to the motion signal, to run the machine as a motor.

$\checkmark$ The DSP generates the ignition signals for the coils driving boards, which performs operation control. This control function is also accomplished by employing position data.

Position detection of such data is essential to the entire control system. Position information is acquired through a Hall-effect sensor and the accurate position and displacement value has been calculated by the DSP. The sensor has been embedded in a Printed Circuit Board, which has been designed specifically for this task. The measured value is received and processed by the DSP and all control decisions have been made based on this provided data.

$>$ Data acquisition has been carried out through SCI and SPI communication protocols and with the aid of a Digital to Analog device. The position signal is accessible analysis and display, for testing and troubleshooting purposes, as well as monitoring system performance.

$>$ A method is proposed and tested to overcome the Electromagnetic Interference (EMI) of the inverter switching with the position sensor signals and has a considerable effect on the input data for the system. 


\section{Introduction and Literature Review}

The OLEA machine is a permanent magnet tubular linear alternator, which is coupled with a free piston internal combustion engine and flexure bearings as shown in Figure 1. This design offers the benefits of a linear permanent magnet associated with a linear free piston engine for efficiency, durability and low cost.



Figure 1. CAD design of linear alternator/engine (WVU proprietary)

Linear electric motors produce unidirectional or oscillatory short-stroke motion. Reciprocating or oscillating linear electric generators transform short stroke oscillatory motion to AC electricity. Electric machines, either linear or rotary, may be designed to operate as motors or generators. Motors convert electrical energy into the mechanical energy and create motion by consuming electrical power. On the other hand, generators convert mechanical energy into electrical energy. In the OLEA, the engine is the energy source and it generates electricity by using fuel and powering the back and forth motion of the translator.

In recent years, linear generators have been increasingly employed for transportation, manufacturing, automation, material processing and generator systems applications [1]. It is notable that the linear generator is advantageous over its rotary counterpart for applications in 
which the available mechanical drive is of a linear motion type. This is because, in these applications, linear generators have higher efficiency, are simpler, more reliable, more compact, and lighter weight.

Reliable standby or emergency generators as a backup power supply are essential in residential households and have several industrial and commercial applications. The backup generator is useful in the event of a power outage within the utility grid. In addition, remote power generation offers the ability to generate electricity where utility service is not available [2], giving areas with no connection to the utility grid, the ability to generate power independently. Standby generators also provide additional electric power in times of peak demand without increasing a facility's electric bill [3]. They can also dispatch excess electricity back to the grid. The linear engine and alternator is particularly useful in fulfilling these tasks due to its compact and lightweight design, allowing it to be utilized as either an on-board setup or a portable power generator.

Linear electric machine designs have been investigated and developed and optimized in recent years. Boldea and Nasar [4], analyzed the permanent magnet linear alternator for both single-phase and three-phase machines. In another research paper, Chen presented a basic design approach for a tubular linear alternator [5]. Wang discussed the design optimization issues of a linear permanent magnet generator coupled with a free-piston engine as well [6].

Different types of linear machine designs are offered in [7], and [8]. A dual piston configuration has been suggested in [8] for linear generators powered by an internal combustion engine. The dual piston configuration is more suitable for high-power applications but is a bit more complex to control. A flat-type permanent magnet linear alternator for free piston linear alternators is proposed in [7] instead of the tubular one. For this application, Wang, suggested a tubular electric machine type since the force of the piston on the translator is high, due to a high level of forces during the combustion process [6]. Thus, forces acting on the translator will be more equally distributed and will reduce mechanical impact on the translator. The effects of the radial and axial magnets for a tubular linear alternator with free piston engines have been studied in [20].

Some other existing publications have analyzed and examined the linear alternator and engine combinations in generating mode of operation while the engine is the primary mover. Combustion makes the translator reciprocate and the linear electrical machine converts the moving part's kinetic energy into electricity. An integrated design approach for the alternator and engine, as the 
prime mover, has been offered. In addition, the interactions between the alternator and engine have been provided in [5]. A Free Piston Linear Generator is introduced in [9], which is being developed at the German Aerospace Center. Mikalsen describes the design of a free-piston engine generator, applicable to electric power generation in large-scale systems and compares them to the conventional engines [10].

There are some papers that analyze and compare linear machines with the conventional machine features. The benefits of a linear free-piston, internal combustion engine over the conventional crank-slider engine include improved efficiency, higher power-to-weight ratio, reduced vibration operation, reduced frictional losses, and multiple fuel capability due to variable compression ratio [11]. Feng studied the properties of the free piston diesel linear generator during the combustion process and compared the combustion of the traditional reciprocating engine with a free piston diesel linear generator [12].

The following papers and [13] investigate the performance of the linear machines through simulation and analyses. Performance and development of a two-stroke, free-piston engine for electrical power generation has been investigated in [14]. Li has presented a model and simulation of a free piston linear alternator and demonstrates that free piston motion is different from that of the traditional internal combustion engines [15]. The effects of various intake pressures, moving masses, ignition positions, and resistance loads are studied.

One important application of the linear machines is in hybrid electric vehicles, where it helps to save more energy and extend their range [16], [17]. Due to the elimination of the crankshaft from the rotating system, the Free Piston Engine (FPE) has more a more compact volume, lower weight, and higher efficiency. Therefore, FPE is typically employed in hybrid electric vehicles [18], [19]. Without the crank and rod bearings, the engine has fewer moving parts, which results in less frictional loss and longer operating life. Moreover, Cawthorne has suggested the design, construction, and testing of a permanent magnet linear alternator and engine combination for use as an auxiliary power unit in hybrid electric vehicles [18].

However, most literature concentrates on linear generator design in steady-state operation and generating mode. There are only a few reports available with detailed investigations on the starting procedures or controlling the machine in the motoring mode operation, such as [3] by Cawthorne and [11] by Djatmiko. 
Zulkifli in [11] proposed a method consisting of two basic principles, mechanical resonance via reciprocation, and electrical motoring via PWM current commutation.

Johansen offered utilizing compressed air, to produce the required motion in [21]. However, this approach requires a separate auxiliary compressor system. If the linear engine does not function as an air compressor, this method adds complexity and cost to the overall system.

Supplying a sufficient force to drive the piston to reach the required compression ratio in one stroke is suggested in [22]. However, this method is only feasible for small engines [23]. On the other hand, Djatmiko suggests to generate a constant motor force in the direction of the piston movement, and gradually achieve the required compression ratio by mechanical resonance [11]. The linear starting mechanism can use the available stored electrical energy or can be mechanically assisted by other inherent mechanisms such as a resonating spring-mass system [11].

The most common method is to run the system as a linear motor to produce linear motion to start the engine [18], [1], [24], [8], and [25]. The linear electric machine operates as a motor during startup and once the engine has proper compression ratio combustion starts and then the electric machine switches to a generator to produce electricity in steady-state operation. The electrical current is generated from machine windings, through interaction between coils and the moving translator magnets that are in continuous reciprocating motion. The electric energy can be stored or consumed by the load. These studies used the coupled linear generator to initiate operation since it is efficient and economical.

Zheng, controlled the alternator to oscillate the piston until steady generating operation. Afterward, the controller converts the AC output of the alternator into suitable DC [26]. Control variables and disturbances are identified, and a control strategy based on the piston position is proposed for a free-piston engine generator in [27]. The detailed information on implementing the controller has been provided by Mikalsen [28].

Jia presents the development of a spark-ignited free-dual-piston engine generator and introduces the design of major sub-systems such as ignition signal generation. The linear machine operates in a motoring mode to start the engine and switches to a generating mode after successful combustion. The main control system generates the ignition signals and the correct ignition timing based on the 
piston position [29]. The real-time displacement data comes from an encoder. In this study, an off the shelf controller processes the position signal and controls the system.

Eid in [24] used an absolute analog position sensor to feedback the position to the controller. The analog sensors are sensitive to electromagnetic noise. In addition, engine control signals are generated based upon the position information. Therefore, a reliable and robust sensor is required to be employed in the overall system.

Some other novel papers discussed the Stirling Engine in the linear machines [30]. Kankam discussed the effects of variations in system parameters on the dynamic behavior of a Free Piston Stirling Engine Linear Alternator system in his paper [31]. Also, Kankam investigated the controllability of the system [32].

In addition, rapid developments of microcomputer controlled power switching techniques stimulated an extensive interest in the permanent magnets (PM) machines [33]. Availability of high energy permanent magnets, powerful and economical microprocessors, and advances in digital control increased interest in linear machines. Advances in DSP technology, which made them more powerful, fast, and less expensive, led to a substantial progress in information extraction concerning the motion and electrical control of the motors.

However, there are not any related works available about details of the control systems of the linear machines specifically the implementation and challenges. This research presents the implementation details and overcomes the position detection difficulties due to the noisy environment.

A start-up mechanism has been implemented to initially start the engine. Afterward, the controller focuses on the engine performance and regulation by adjusting the combustion timings.

The controller starts the OLEA from standstill state by an open loop mechanism. Subsequently, the controller starts the closed loop control mode when the position information is available for the controller. The controller runs the OLEA for a few seconds with a closed loop control system according to the translator position information. The start-up mechanism is a transient state to achieve the required stroke for the engine to start operating the OLEA in generating mode. Afterward, the controller focuses on controlling the engine to operate in an efficient way by 
generating the ignition signals based on the translator position data, which plays an essential role in engine functioning.

Control of a single-phase linear machine with implementation details has been provided in this research. This research focused on instrumentation and control of a linear tubular permanent magnet alternator combined with a two-stroke, free-piston internal combustion engine into a single unit. The entire process has been implemented and tested on the machine and a functioning linear generator is the result.

\section{OLEA System Description}

\subsection{OLEA Introduction}

This project is part of ARPA-E funded GENSETS program. The goal is designing and building a micro power generator that operates on natural gas.

A considerable amount of energy is wasted at generation, transmission and distribution from the power plant to the customer. The proposed OLEA system is a compact system which generates power using the natural gas pipelines inside homes. Therefore, the GENSETS program is looking for development of $1 \mathrm{KW}$ electricity generators with high efficiency, long life (10 years), compact size, and low cost, unlike the systems that are already available on the market.

The WVU team is developing a generator for residential applications. The generator is a permanent magnet linear tubular electric machine. It is driven by a two-stroke, free-piston internal combustion engine. Using springs in this system stabilizes the generator stabilizes the operation. In addition, the free-piston design offers fewer moving parts and less frictional losses.

This development can help households and businesses become self-supporting in case of power outages. The generator can also be used as a backup system in islanding mode since it can be installed independently from the network. In islanding mode, in conjunction with battery storage, the generator can transmit the excess electricity back to the grid. Also, higher efficiency decreases the overall energy consumption and therefore less air pollution is generated. In addition, this system could offer lower electricity bills with continuous operation for an extended period of time. 


\subsection{OLEA Description}

The OLEA machine is a linear mass-spring system which runs at its resonant frequency. The system starts to run as a motor by the input electric power. In motoring mode, the machine oscillates and creates a reciprocating motion to achieve appropriate engine compression ratio. When the machine reaches the desired compression ratio, the engine starts and generates power by getting fuel.

The linear tubular machine is required to operate as a motor to start the OLEA. This is achieved by employing a power electronics converter (inverter) to drive the motor and run it at the system (mass-spring) natural frequency which is also the system resonant frequency. After reaching the specified displacement and the desired engine compression, OLEA starts and the machine switches

to the generating mode. Input power to the inverter is switched off and engine generates electricity. A control and sensing systems is essential for the operation of OLEA both for engine start-up and during operation in generating mode.

There are challenges in implementing the above starting technique in this resonant system. The OLEA system is a coupled electromechanical system. In the electrical side, there is essentially an RL circuit with back emf running in steady-state at the resonant frequency. On the other hand, the mass-spring is also mechanically oscillating at the same frequency. In this system, the forces due to mechanical oscillation dominates. Since the linear electric machine is primarily designed for power generation at $1 \mathrm{KW}$ for a specific voltage, operating the machine as a motor does not produce forces to overcome dominant mechanical oscillation in an open-loop control setting. Therefore, a feedback loop control has to be employed so that the machine operates at high displacement to achieve the desired engine compression necessary for starting.

A Phase Locked Loop has been selected for a feedback control to generate controlling signals for the system based upon the requirements. The controller is a digital signal processor. The input data for the PLL control technique is instantaneous position information which is provided by a Hall effect sensor. An inverter has also been designed and built to drive the linear machine as a motor. 


\subsection{Linear Tubular Brushless Permanent Magnet Electric Machine}

Linear electric machines in some applications have some significant advantages over rotary machines including simplicity, high dynamic performance, compactness, improved reliability, and high efficiency. The inherent high efficiency is due to operation without any need to convert the rotary motion to the linear. Since the linear machines are able to form linear and reciprocating motion without elaborating the arrangement of intermediate mechanical gears and transmission systems. They gained more popularity and are used widely in different direct applications of linear motion such as lifts, elevators, and transportation [24]. In applications where rotary motion is mechanically converted to linear motion, through an elaborate arrangement of crankshafts, ball screws, etc., eliminating the mechanical losses can significantly improve the energy efficiency. Simultaneously, the advent of permanent magnets, with high energy density, and power electronic converters with digital controllers resulted in high-precision energy-efficient drives to be developed for linear motion control and power generations.

Furthermore, the PM machine makes it unnecessary to supply current to the translator moving parts through slip rings or commutators for its magnetization. The stator armature current is only responsible for producing torque, unlike in DC motors. Moreover, PM machines inherently have higher efficiency since they do not have rotor losses and the need for translator excitation. Unlike wound-rotor synchronous or DC machines, PM machines do not need regular brush maintenance as well, which eliminates downtime due to maintenance of the brushes. The most important advantage of the brushless PM machine over other conventional ones is their higher efficiency [31]. A key point in brushless PM machines is the field coil, its associated dc power supply, and slip rings/brush commutators have been replaced with permanent magnets [34].

The linear brushless PM motors are high-efficiency machines with simple control systems and high dynamic performance. They are commercially employed in different applications such as manufacturing automation, electrical power generation, transportation, healthcare, and household appliances. 


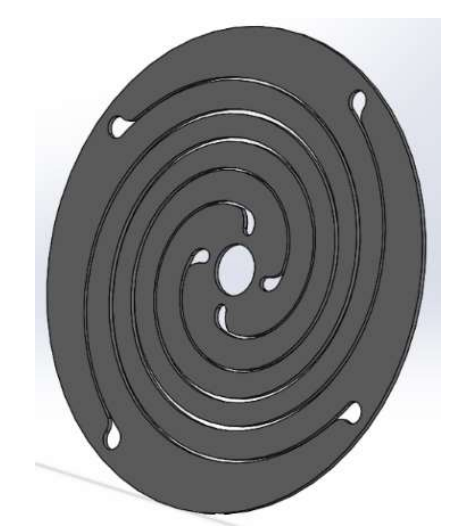

Figure 2. Flexure bearing used in OLEA system

\subsection{Springs in the OLEA}

The OLEA system utilizes flexures that act both as bearings and spring as showing in Figure 2. In a mass-spring system, the resonance frequency is a function of the mass and stiffness of the spring. A linear machine has a linear displacement when it is running. In order to generate more displacement, more energy is required. The maximum displacement of the alternator is achieved when the system resonates at its natural or resonant frequency. Maximum transfer of energy occurs by oscillating the system with the natural frequency. In other words, energy resonance always occurs when applied frequency is equal to the natural frequency. In addition, a small amount of energy is needed to overcome losses such as friction, windage, core, etc. to run the system at its natural frequency.

The reason to incorporate springs into the system is to run at higher frequencies. In case of the alternator, operating at a higher frequency will reduce its size, increase its power, and make it on par with its rotary counterpart. In case of an engine, operating at a higher frequency will increase its power and efficiency. The key is to select flexures to raise the resonant frequency as high as mechanically possible. Higher spring constant means more challenges during startup overcoming mass-spring oscillating forces to achieve the desired displacement for engine compression. 


\subsection{Resonant Frequency}

Although the machine can be run in any frequency, the desired frequency value is the resonance frequency to get the maximum displacement. In an ideal mass-spring oscillating system, the resonance will be achieved by varying the input mechanical force's frequency to match the natural frequency of the system. Moreover, the total input energy must overcome damping losses in the system. In this system mechanical force is generated by the linear motor by pulsing the inverter drive. The inverter frequency is adjustable by varying the input voltage pulse frequency set by the controller. In open loop mode, the controller is set to generate driving pulses with the resonant frequency and the machine oscillates at its natural frequency. In a decoupled electro-mechanical resonant system an open loop control set at natural frequency should result in system's resonance frequency automatically.

Briefly, the position information is feeding to the controller continuously during the run. The controller generates a 25 percent duty cycle pulse at every position maximum and minimum point to drive the motor. The frequency of the operation is self-adjustable to the resonance frequency. The inverter's task is to direct the current into the machine phase windings in a proper direction.

\subsection{OLEA Engine}

The generator is developed based on a two-stroke, free-piston internal combustion engine. This free-piston design in comparison with the slider-crank models eliminates the crankshaft. This results in a compact design with fewer moving parts and less frictional losses.

A two-stroke engine completes a power cycle with two strokes of the piston - compression along with the combustion and exhaust along with the intake.

Two-stroke engines have a higher power relative to their size. So, two-stroke engines can be more compact and lighter.

\subsection{Position Detection}

To drive the machine as a motor for startup, voltage is applied according to the translator's position. Translator position information at every instant is required to run the machine to perform 
the electronic phase commutation in a proper manner. A linear single phase PM machine commutes and controls the current at two points in each cycle. Applying the driving voltage is supposed to be synchronized with the alternator movements. Therefore, a position sensor is required to provide the high-resolution real-time position data to control the machine and ensure the supply of suitable excitation signals.

In addition, the machine controller energizes the stator winding in a sequence to commute and oscillates the machine. The knowledge about the exact position of the translator at any time is the key point to determine which winding should be excited. Furthermore, accurate position information is needed for ignition timing to run the engine. Therefore, a position sensor is required to be installed on the machine to sense and track the motion and displacement.

\subsubsection{Potentiometer}

Initially, a linear potentiometer was installed to measure the position and to display on an oscilloscope. The potentiometer is a variable resistor, which consists of a strip and a wiper as shown in Figure 3. Linear Sensor Thin Pot and Figure 4. Sensor Wiper. The linear position sensor is a $3 \mathrm{M}$ pressure sensitive adhesive tape which is a $10 \mathrm{~K}$ Ohm variable resistor with a $25 \mathrm{~mm}$ length. Also, the wiper is a force actuator that provides a required force of 1 Newton to 3 Newtons. The strip has three pins that the two on the sides should be connected to reference voltages, and the middle one is the variable voltage which represents the position based on where the wiper contacts the strip. The wiper should be installed above the strip and slightly touch it. Either strip or wiper should be mounted on the moving part of the machine, and the other one should be fixed on a still place.

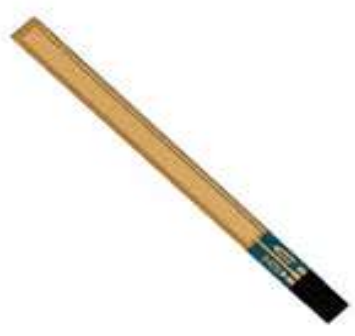




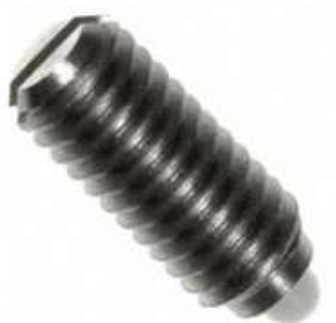

Figure 4. Sensor Wiper

The main advantage of the potentiometer is that it is easy to install, and makes the system simple. However, it is prone to noise and needs maintenance frequently. The strip and the wiper also can break down in a short time of operation due to wear and tear. Although the potentiometer enabled capturing position which in this OLEA system is a sine wave motion of the translator, due to its high sensitivity to noise, high maintenance and low operating lifetime, a more reliable and robust method of data acquisition was required.

Since for this OLEA application all the control decisions and control signal generations are based on the position data provided by the position sensor, an incremental magnetic encoder would satisfy all specification mentioned previously and is selected as a more robust and reliable sensor. However, the position value is not available directly from the sensor in a continuous manner and its outputs need to be processed to measure the position value out of three output signals of the sensor that will be explained next.

\subsubsection{Hall Effect Sensors}

For this application, Hall effect sensors have many advantages over the analog potentiometers. Potentiometers are too sensitive to noise and their quality degrades with their operating time. On the other hand, Hall effect sensors have no contacting parts and do not suffer surface bounce. Furthermore, friction or vibration does not affect the output results, and a long lifetime of operation can be expected from Hall effect sensors. In summary, the main benefits of Hall effect sensing technology are high reliability, small size, low cost, wide operating voltage ranges, a variety of output options, flexibility, and ease of assembly. These advantages extend the use of this family 
of sensors in the marketplace. Moreover, Hall effect sensors can operate at high frequencies, or can be employed under severe environmental conditions since the sensor is in a sealed package. On-chip techniques have been incorporated to the most recent packages of Hall effect sensors to overcome problems of sensor sensitivity to the potential external environmental effect. However, one main disadvantage is implementation in which output signals need to be processed to obtain a position value.

The Hall effect devices work based on the Hall effect theory that refers to the occurrence of a measurable voltage across an electric current-carrying conductor transverse to the current, while the conductor is kept in a magnetic field. In other words, when an electric current flowing through a conductor, which is influenced by a magnetic field, a transverse force is created across a conductive material and it is known as the Lorentz force.

The sensors are normally mounted on a printed circuit board and are placed on the stationary part of the machine, which is the stator. The magnetic field moves back and forth relative to the sensor to actuate the device. In most cases, a Hall effect magnet is embedded onto the rotor in addition to the motor main magnets, to simplify the process which creates the same effect. The fixed magnet provides the capability of aligning the sensor with rotor magnets to gain the best operation.

Burger, Besse, and Popovic [35] have utilized a three branches vertical Hall sensor to observe the position data in three-phase machines. The principle of this sensor is a miniaturized magnetic angular encoder based on 3-D Hall sensors.

Hall effect sensors are divided into two categories, analog, and digital encoders. Analog encoders provide a continuous linear output voltage values proportional to the magnetic field to which it is exposed. The output is taken directly from the output of the operational amplifier.

Digital output sensors, on the other hand, have just one of two states: ON or OFF. A Schmitt trigger with built-in hysteresis has been added to the analog devices and is connected to the op-amp. The Schmitt trigger compares the output of the differential amplifier with a reference. When the magnetic flux (the amplifier output) passing through the sensor exceeds a pre-set value, the output switches quickly between its OFF condition to an ON condition. By the means of the hysteresis, which depends on whether the sensor is being turned ON or OFF, there is no oscillation in the output signal and results in jitter-free switching. 
A Schmitt trigger is an electronic circuit with two stable states (bistable). When the input rises above a certain threshold, the output increases to the steady maximum value. Conversely, when the input falls below a second certain threshold, the output decreases close to zero.

Hysteresis is the system in which the state of a system is dependent upon its history and the way it has changed in the past.

From another point of view, encoders can be placed into Absolute or Incremental encoders types. Absolute encoders are capable of providing unique position values that are generated corresponding to a unique coded element.

However, Incremental encoders send an output signal each time they pass a certain amount of distance. By powering on the encoder, it begins counting from zero, regardless of its previous position. Incremental encoders are suitable for applications such as frequency, speed, direction, and position monitoring. They are more cost-effective and less complex than absolute encoders. An incremental encoder outputs a displacement or change in distance, not the absolute value of the position until it is provided with a reference point from which it measures. But an absolute encoder outputs its position without the need for any reference information or movement.

Incremental encoders are available with higher resolution and accuracy compared to the analog ones, while the analog encoders simplify the system. Therefore, a linear incremental encoder with desired speculations has been selected to achieve goals for this application.

\subsubsection{Position Detection by a Linear Incremental Magnetic Encoder}

Based on our application, an RLS RLC2HD Miniature Linear PCB Level Incremental Magnetic Encoder has been selected to be employed as a position sensor for data acquisition and to locate the alternator translator in real time. It sends out three digital output signals to determine the relative position with 5 Micrometers accuracy.

The above magnetic encoder was selected as they are robust and durable, with a compact design for installation in small spaces, and they are mechanically simple and economical. 


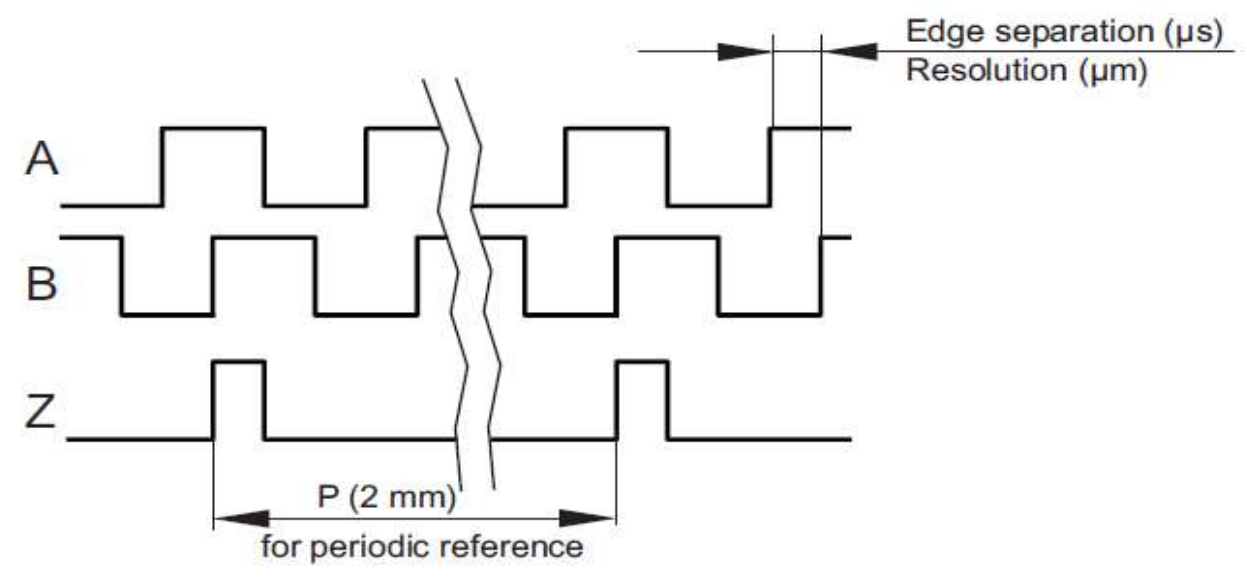

Figure 5. Timing Diagram - Incremental, Periodic Reference Mark

The encoder chip, mounted on a Printed Circuit Board (PCB) board, should be embedded above the magnetic strip. The magnetic strip has magnetic poles for every $2 \mathrm{~mm}$. It is attached to the machine shaft that is moving and the encoder is installed fixed, on the end cap above the magnetic strip.

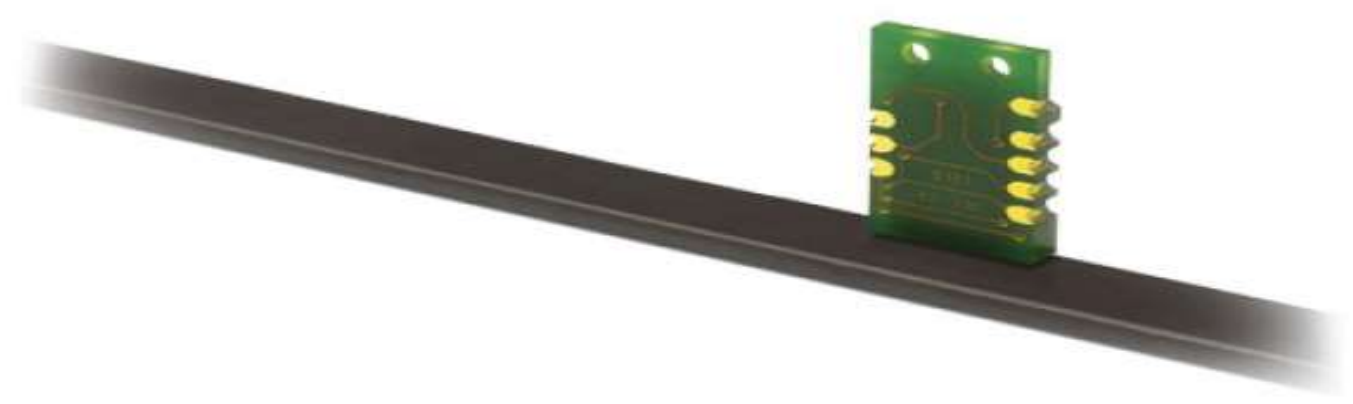

Figure 6. Linear Incremental Magnetic Encoder

The sensor needs to be supplied by a $5 \mathrm{~V}$ power supply, and output signals are two square waves and an index pulse. The voltage level of the signals for their high level is $5 \mathrm{~V}$, and $0 \mathrm{~V}$ otherwise. However, the input voltage range of the DSP is within 0 to $3.3 \mathrm{~V}$ range. Since the three output signals should be fed to the DSP for position calculations, a step-down voltage converter is 
required. A Printed Circuit Board has been designed to embed the encoder and the voltage level shifter (MC14504B, Hex Level Shifter). The designed PCB provides the connections to the DSP for proper communication. The board is compact and durable in terms of having more stable wiring.

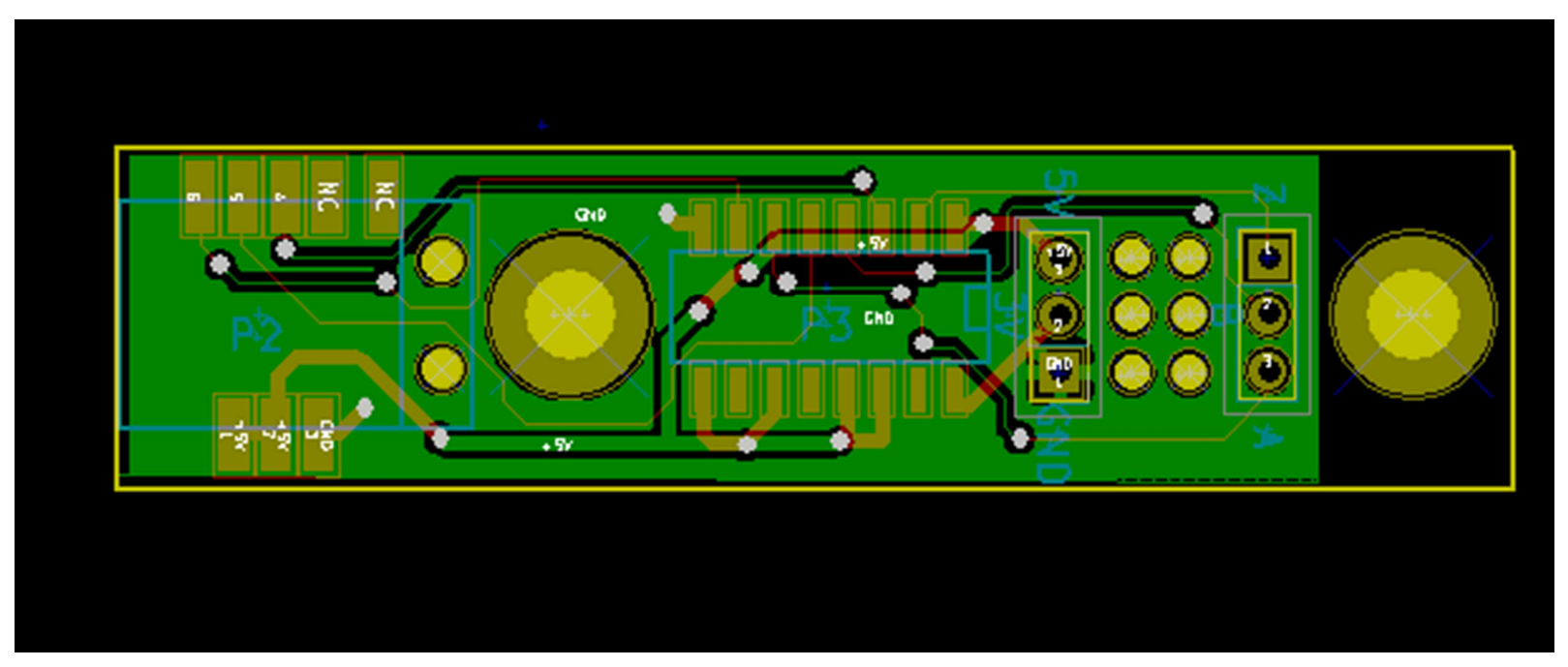

Figure 7. PCB Design of the Sensor Board

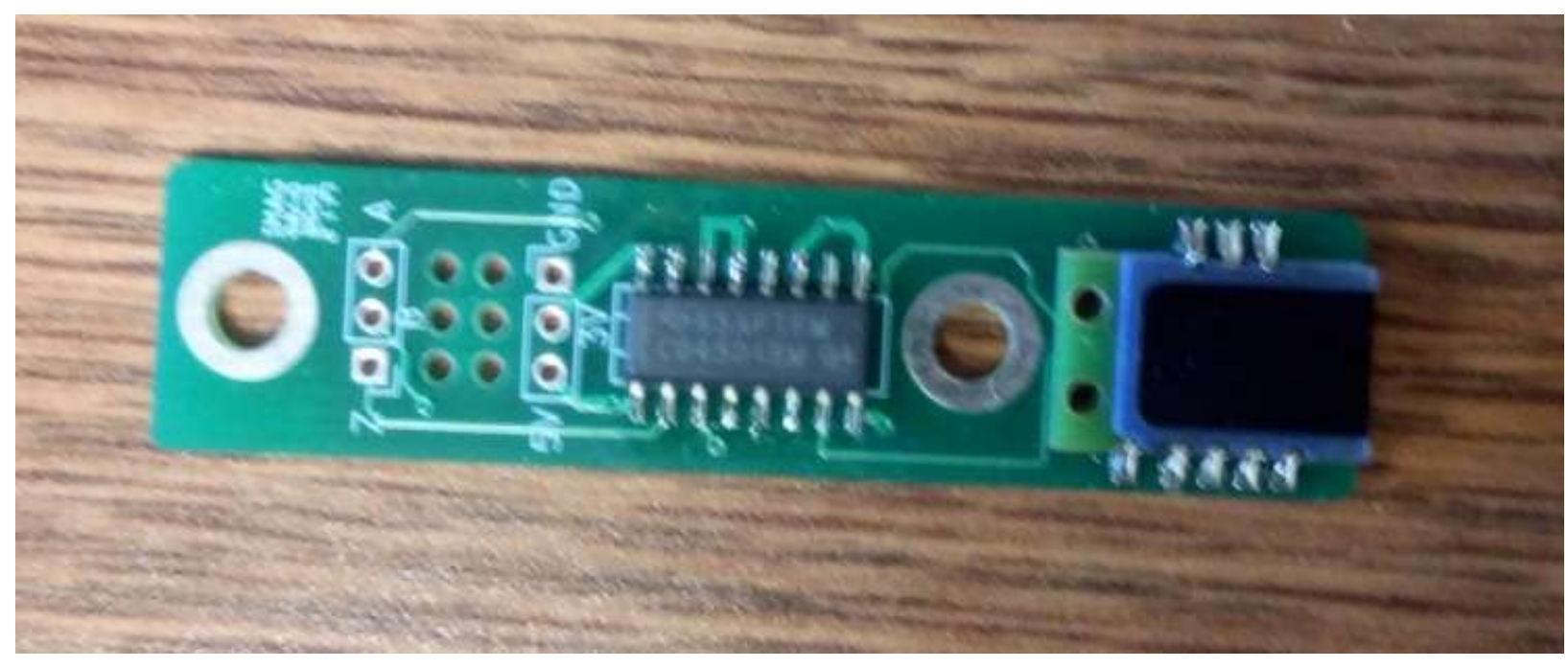

Figure 8. Position Sensor Mounted on the Printed Circuit Board

The incremental encoder provides three output signals, namely A, B, and Z (or index). The two square wave signals, $\mathrm{A}$ and $\mathrm{B}$, have a 90 -degree phase shift. This phase shift is an indicator to 
detect the direction of movement, which indicates if the translator is moving forward or backward. If $\mathrm{A}$ is ahead of $\mathrm{B}$, the motor is moving forward and if $\mathrm{A}$ is behind $\mathrm{B} 90$ degrees, then the motor is moving in reverse direction. The DSP counts every rising and falling edge of A and B signals. Every cycle of A and B indicates 5 Micrometers displacement. In addition, the third signal, Z pulse, occurs every 2 millimeters distance at each pole. Therefore, the $\mathrm{Z}$ pulse is the resetting point and also can be used for absolute position calculation. Hence, by manipulating these three signals by the DSP, absolute position information is available in an online fashion with 5 Micrometer accuracy.

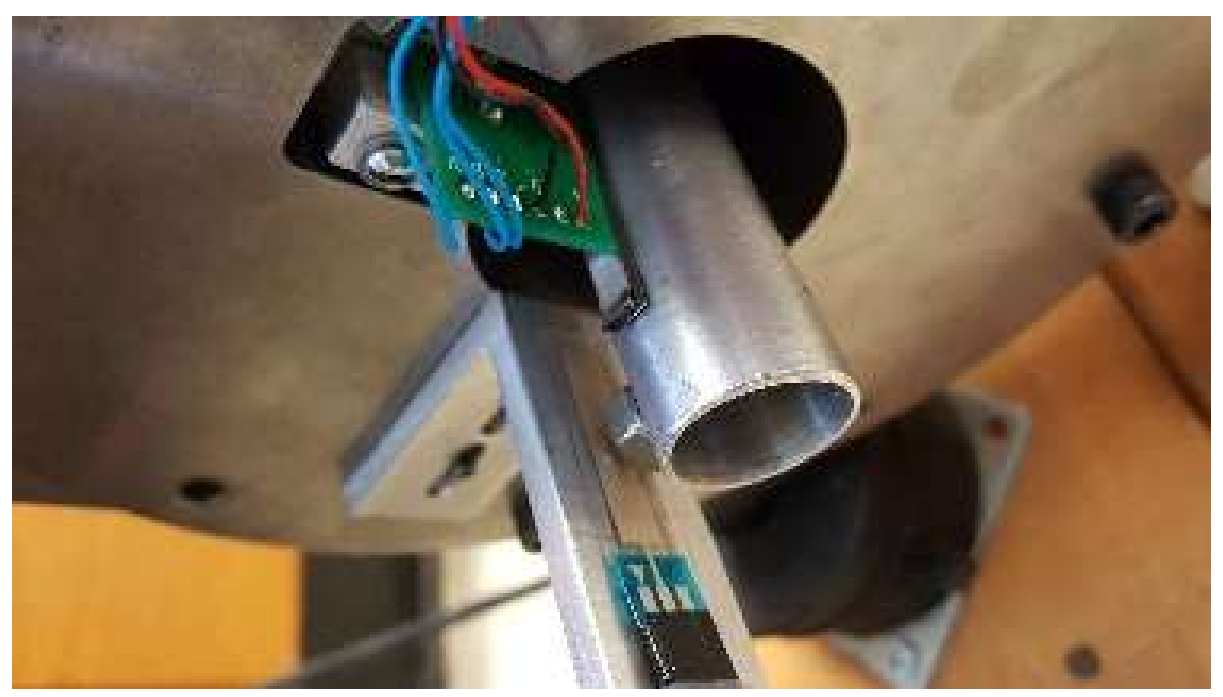

Figure 9. Sensor Installed on the Machine 


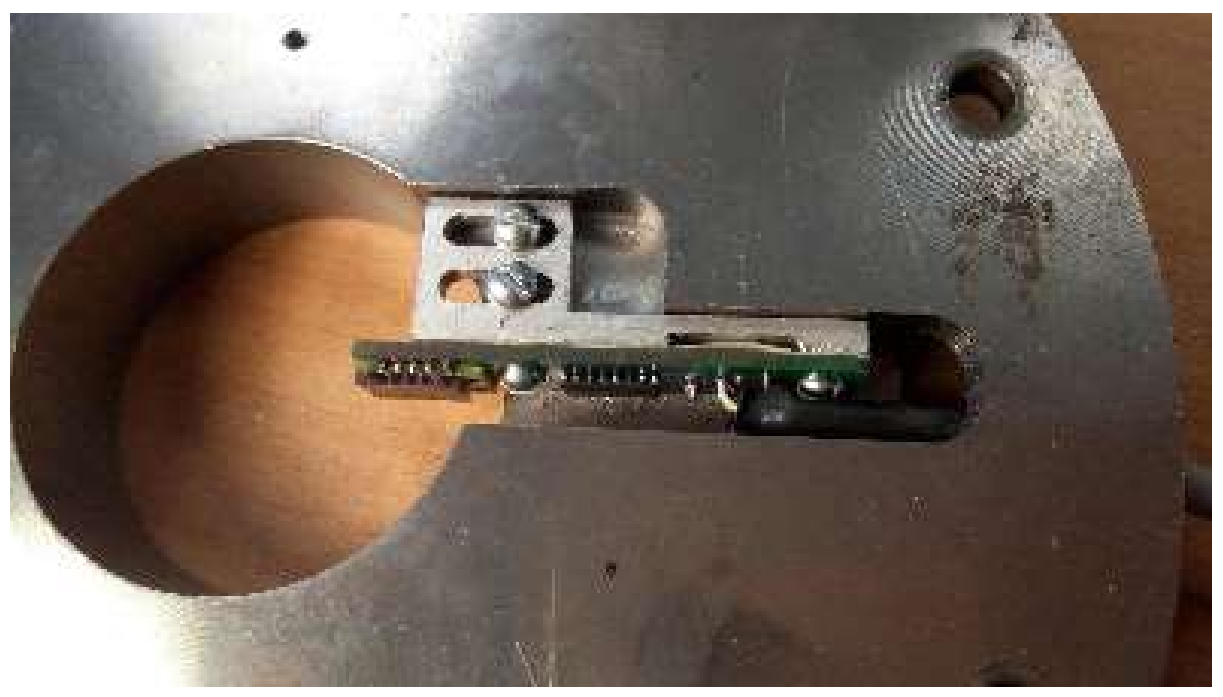

Figure 10. Sensor Installed on the End Cap

\subsection{H_Bridge Introduction}

An inverter is power electronics hardware that converts DC power to $\mathrm{AC}$ power and commonly used to drive brushless DC machines by directing current to appropriate phase windings. A gate signal comes from the controller to switch on the appropriate semiconductor device. The turned on semiconductor devices create a path for the current to flow.

An H_Bridge is an electronic circuit with four switches, with the load on its center that can reverse the current through the load. By turning the two sets of switches on and off, the load current flow can be controlled. In this way, a single current source can be employed and driving current in two directions can be accomplished.

H_Bridge is used as an inverter to execute the phase commutation and reverse the current direction for the single phase linear machine.

When switches S1 and S4 are turned on (see Figure 11. H_Bridge General Block Diagram), and switches S2 and S3 are turned off, the left end of the motor will be connected to the power supply, while the right end is grounded. Therefore, current flows in a direction to energize the motor in the forward direction. On the contrary, when switches 2 and 3 are turned on, and switches 1 and 4 are turned off, the right end of the motor will be connected to the power supply, while the left end 
is grounded. Consequently, the current flows in a direction to energize the motor in the backward direction.

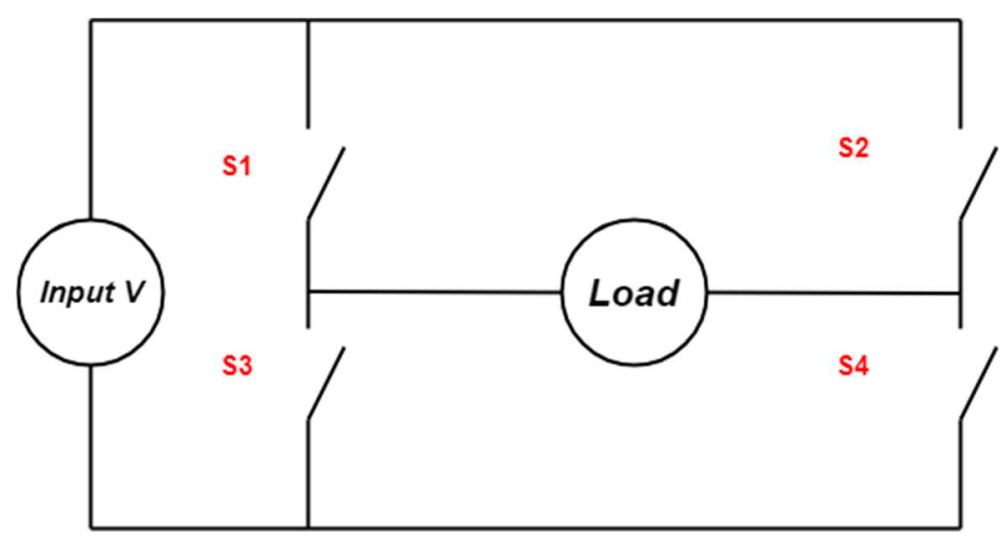

Figure 11. H_Bridge General Block Diagram

\subsubsection{Inverter Operation}

The electronic phase commutation is the process of switching current flow through motor phases or changing the direction of current flow and is carried out by the means of an inverter that supplies the machine terminals.

The position sensor is mounted on the translator shaft and sends out the position information to the controller. The controller triggers the inverter switching actions simply based on position data to ensure that the driving signal is in phase with the machine motion signal.

The sequence of conducting terminals is maintained by the discrete switching events. In case of driving the machine with a pair of pulses, there are two switches that lead the current at every moment, one from the upper side of the inverter which is connected to the power supply, and the other one from the lower side which is grounded.

\subsection{Controller}

The controller is the main central processor for commanding the system. It receives the input data, processes them, and sends out the desired signals. 
A Texas Instruments (TI) TMS320F28335 Delfino Microcontroller experimenter kit has been utilized to function as a controller. It is a high-performance 32-Bit CPU (TMS320C28x), up to 150 MHz (6.67-ns Cycle Time), 3.3-V I/O design microcontroller. The DSP has 12-bit Analog-toDigital Converter (ADC), and different communication protocols, including Serial Communications Interface (SCI), Serial Peripheral Interface, and Inter-Integrated Circuit (I2C) with 88 GPIO pins. The experimenter kit has added capabilities, such as XDS100v1 USB-to-JTAG debug probe, which enables real-time in-system programming and debugging. It has also header pin access to most major digital IO and analog inputs, and a breadboard area for customizable prototyping. The board power can be provided by a USB cable or a $5 \mathrm{~V}$ barrel supply. In addition, the experimenter kit, 2010 C2000 Teaching ROM, was built around the similar TMDSPREX28335 kit and can serve as a good reference. Moreover, a MathWorks Embedded Coder support is included. The user interface, to interact with the hardware and program the DSP, is Code Composer Studio (CCS). ControlSUITE also provides the device drivers and example projects by the support team. Code Composer Studio is an integrated development environment (IDE) for C2000 32-bit real-time control Microcontroller Units (MCU) and Embedded Processors. CCS consists of different tools to develop and debug embedded system applications and includes an optimizing $\mathrm{C} / \mathrm{C}++$ compiler, source code editor, project build environment, debugger, and many other features. Code Composer Studio combines the advantages of the Eclipse software framework with advanced embedded debug capabilities from TI.

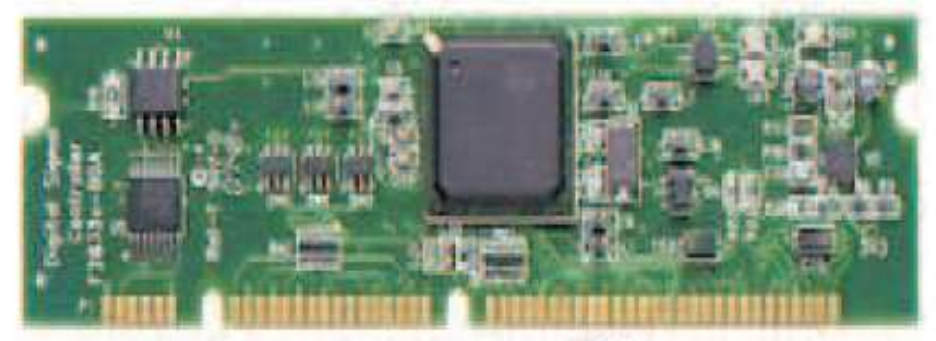

Figure 12. F28335 Delfino Family Control Card 


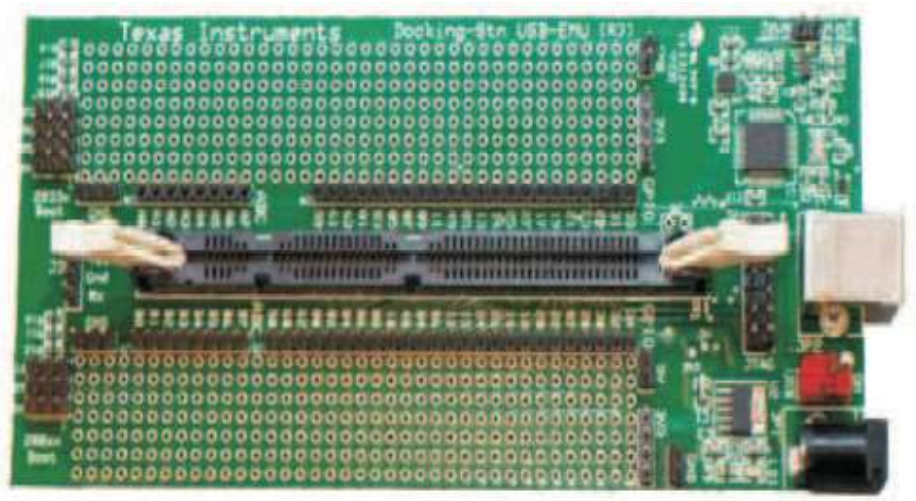

Figure 13. Experimenter's Kit USB Docking Station

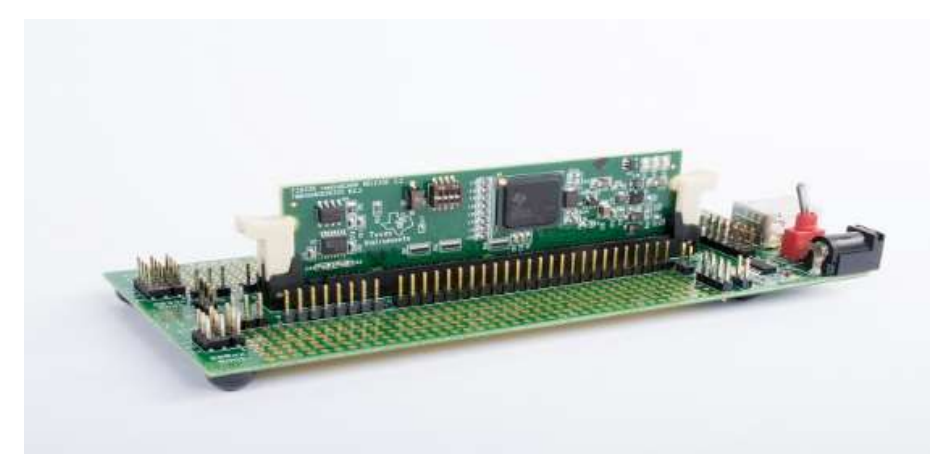

Figure 14. DSP Experimenter Kit (C28x CPU Generation)

DSP runs the machine in an open-loop mode with a single pulse in each cycle. After a few seconds, when the motor reaches a pre-specified constant frequency and displacement, it switches to the PLL mode. In the meanwhile, the DSP reads the position data, provided by the incremental encoder through three designated pins. Based on the provided data from the sensor, and the developed algorithm, DSP determines the timing to set or clear the output. After switching into PLL mode, the controller waits for the motor to oscillate to reach the resonance frequency before pulsing. In that state, motor flexure extension reaches its maximum displacement that results in maximum engine startup compression ratio. Now, the controller can start the engine, and let it run the machine in generating mode. At this state, the controller is responsible for commanding the ignition signals to the engine. It is very important to start the engine at the maximum displacement as the combustion can only happen at a certain displacement/compression. 
Simultaneously, the DSP is supposed to handle other input and command signals, through the $\mathrm{ADC}$ and interrupt pins. It also is responsible to calculate the position/displacement, and then communicate them using SPI protocol. The communicated data can be visualized by an oscilloscope or get logged in a computer.

The controller role in running the entire system is first starting the machine from standstill by an open loop signaling. Then, it switches to the PLL mode and oscillates with the resonance frequency for a while. At the moment the displacement reaches a specific amount to establish compression, the engine starts taking over running the machine, the PLL signals for motoring mode turns off and the controller duty is commanding by generating the ignition signals in generating mode. The combustion occurs at a certain displacement, that moment is detected, the motoring mode stops and generating mode starts.

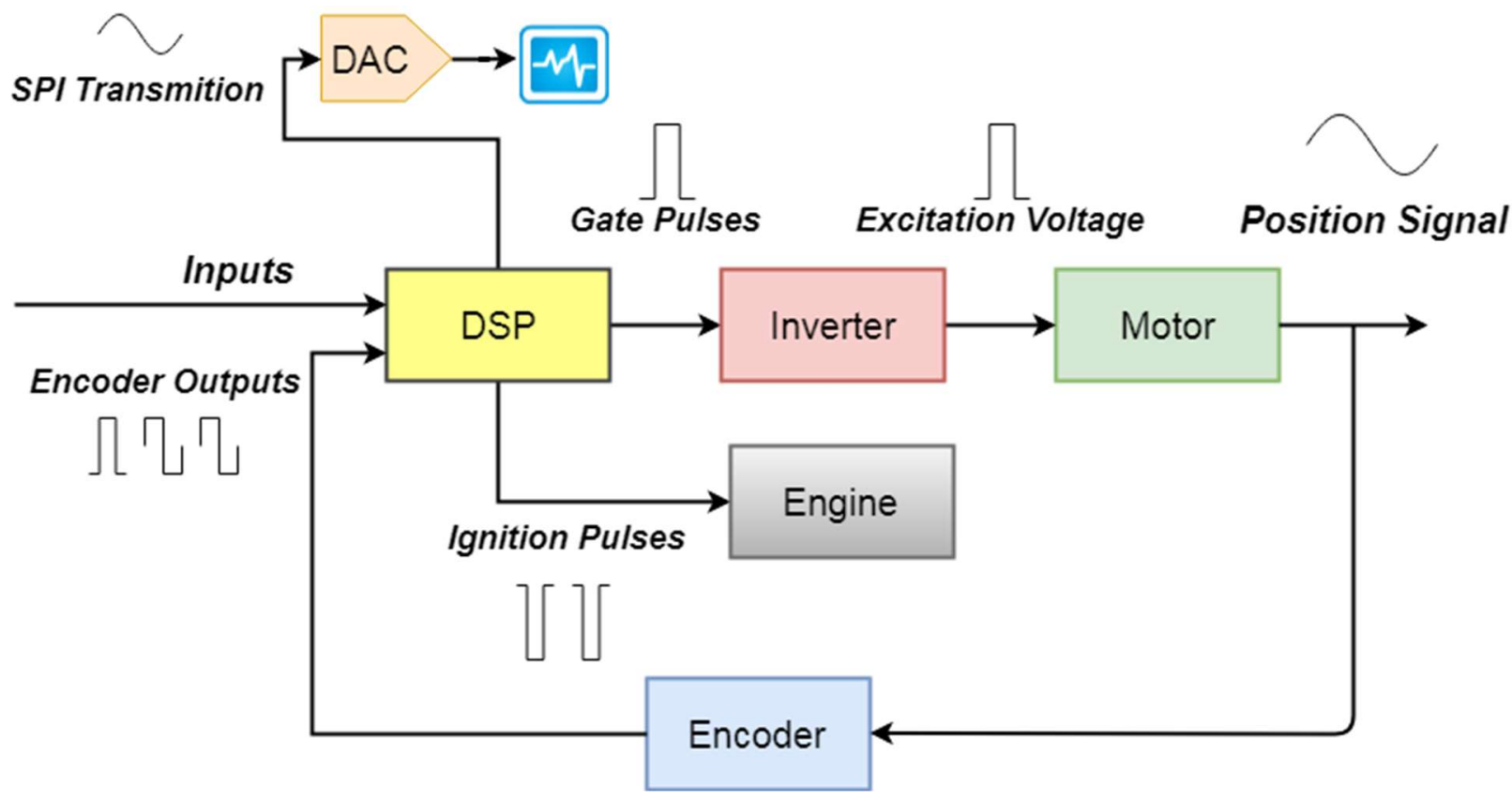

Figure 15. Controller Block Diagram

\section{OLEA Start-up}

Optimum piston speed and engine compression pressure are essential to be regulated to start an internal combustion engine. In the slider-crank engine, the motion is forced to keep the same Top 
Dead Center (TDC) position in every cycle as the crankshaft controls the piston motion. Then the TDC is fixed and the resultant pressure which is related to the TDC remains fixed. Thus, to start the engine, optimum speed is the only factor to ensure. In contrast, in a free-piston linear engine, the translator as a moving part does not follow a fixed displacement and has no fixed TDC. In the free-piston engine, the piston motion is controlled by the instantaneous sum of the pressure and load forces acting upon it the translator and is influenced by the progress of the combustion process. Therefore, the piston movement is coupled to the forces and combustion pressures. Consequently, the translator's displacement amplitude is supposed to be controlled to reach the required compression pressure.

The pressure in the cylinder after closing the exhaust port is a major force that the piston needs to overcome. In slider crank engines, the crankshaft pinion is engaged with the flywheel's teeth and it turns as the flywheel is turned. Thus, the starter motor pushes the piston up into the cylinder to turn the crankshaft during start-up. Due to the slider-crank configuration, a low force is required to start the motor to overcome the compression pressure. On the other hand, the entire force must be provided by the starter motor in the direction of linear motion, opposite the resistive dominated compression force. Since there is no crank-slider configuration or mechanism to reduce the starting force.

In conclusion, the delivery of the starting force and providing the optimum compression pressure indicates the lack of a start-up mechanism for the engine.

The engine is not capable of supplying a sufficient force to reach the required compression ratio, in one stroke. The thrust force supplied by the linear alternator is limited by the peak current in the motoring mode. Thus, a motor force lower than the one stroke force, in the direction of the piston movement, should be generated to achieve the required compression ratio by mechanical resonance during the motoring mode. Therefore, the displacement grows and reaches the required value with the sufficient compression ratio for ignition. At this stage, the engine starts and the motor is switched off.

The Internal Linear Combustion Engine at FPE is at the TDC or BDC before the operation. A force is needed to move the Internal Linear Combustion Engine for the first few cycles until steady-state oscillating operation achieved. The fuel should be compressed to achieve the required compression ratio at the top dead center. Then the combustion process starts and the compressed fuel ignited. 
A starting method is needed to create a linear motion with the minimum starting speed for the linear generator.

The linear generator operates in motoring mode to provide the required force and linear motion for the engine. A controlled voltage pulse with specific value feeds the motor and the inverter controls the current direction through the motor windings to fulfill this. The input signal to the machine is controlled by a position feedback that commands the inverter gate switches. Therefore, the engine motion is controlled until the engine is able to oscillate in a steady-state.

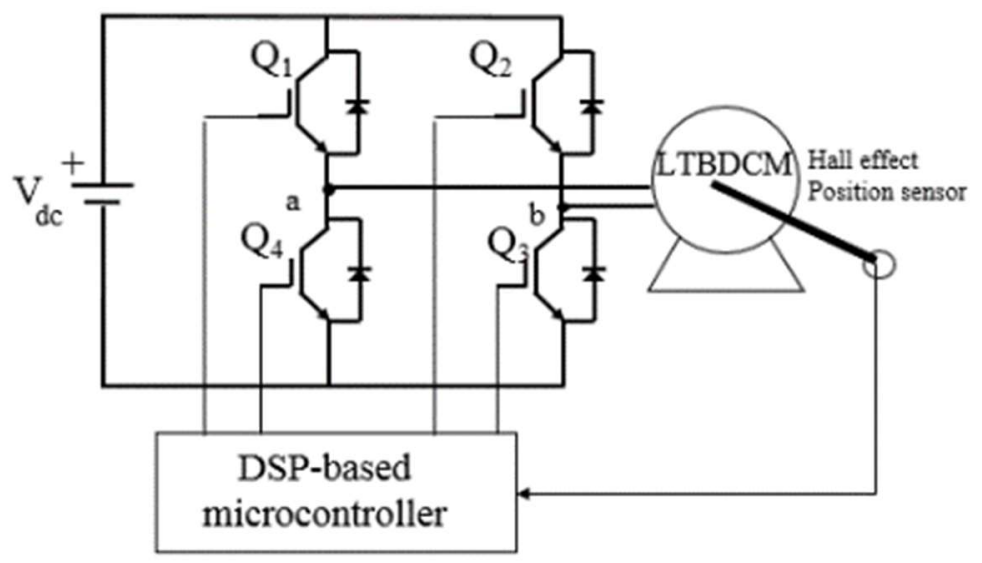

Figure 16. Start-up System in Motoring Mode

To start the system as a generator, it is necessary to reach the start-up displacement for the engine to start running. To get the maximum displacement, the linear machine needs to oscillate at its natural frequency and the driving voltage is synchronized with the alternator motion.

To achieve these requirements, a start-up mechanism is developed which will be explained in details in the following sections. Moreover, a hardware design is necessary to transfer the control signals to the machine and provide the essential voltages and currents to start the machine. The inverter is designed and built to supply the motoring current and voltage. 


\subsection{Inverter Schematic Design}

Component selection and more specifically selection of the switching elements is the first step in the inverter design. There are many factors to consider for switch selection, but generally, operating current and voltage, operation conditions, and the switching frequency must be considered.

Four Insulated-Gate Bipolar Transistors (IGBT) as four switches were selected for this application based on their specifications. The IGBT is an N-channel ultra-fast switching IGBT STGE50NC60WD with 50A continuous Collector current and 600V Collector-Emitter voltage. The selected IGBT features a high current capability, high-frequency operation, and very soft ultrafast recovery antiparallel diode, which makes it a good choice for our application. Moreover, its type of packaging has an adequate level of heat dissipation since it has a large packaging compared to the other IGBT packages. The IGBTs has two main states when operating as switches: on and off. They have fast and smooth turn on and turn off waveforms.

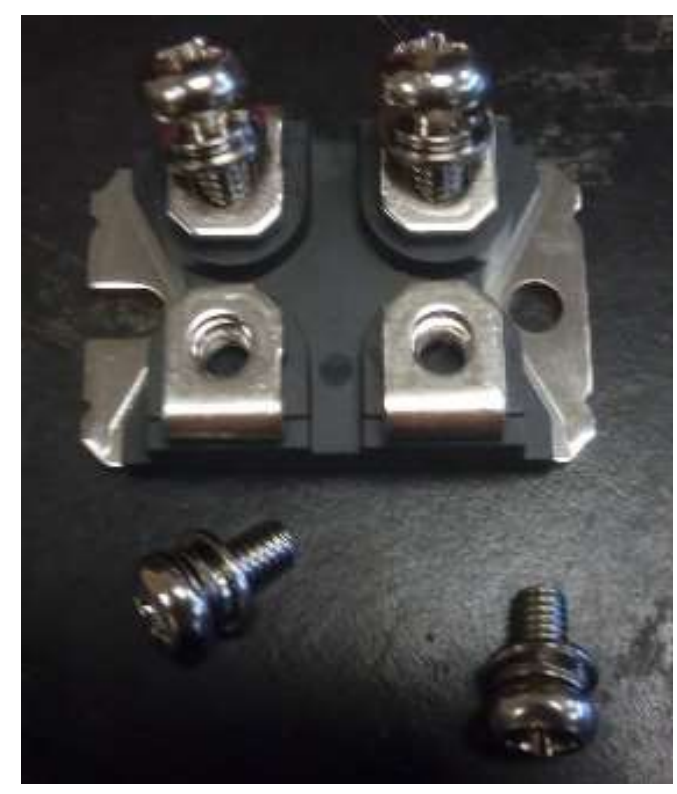

Figure 17. IGBT STGE5ONC6OWD

The antiparallel diode is one of the important elements of designing an inverter. Since the load is a motor which is an inductive load, when the switch turns off, the current of the inductor continues 
flowing and it continues its path through the anti-parallel diodes. Therefore, the diode gives this current a path to flow back to the source when a switch interurpts the current. On the contrary, without an antiparallel diode, the inductive current ends instantly and generates a high voltage peak, which can burn out the circuit. Most IGBTs have built-in the antiparallel diode in their packages.

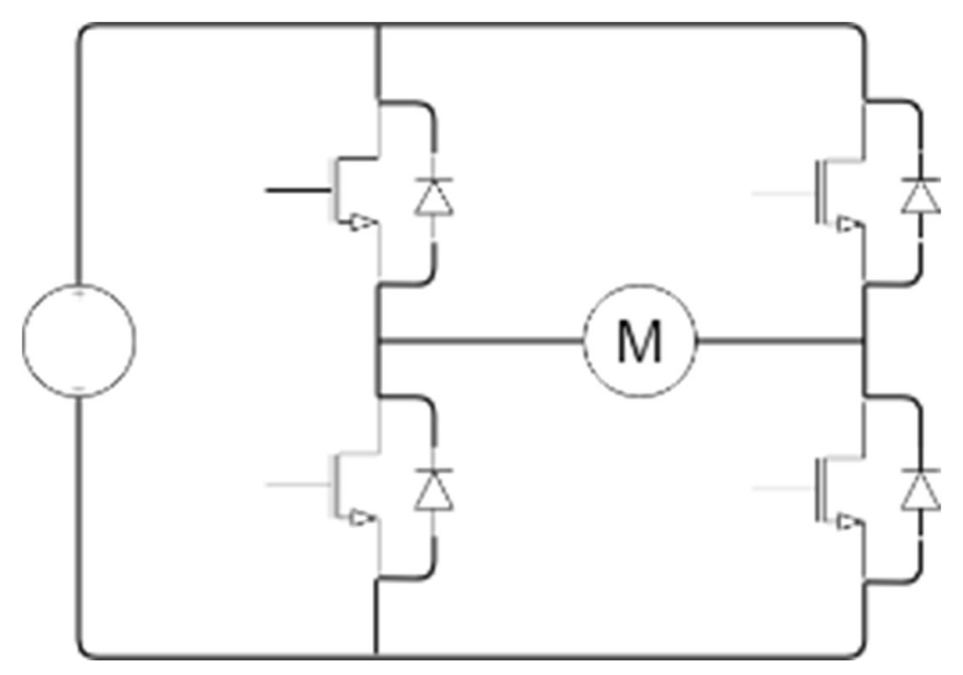

Figure 18. The Antiparallel Diode Role in Conjunction with the IGBT

To drive IGBT gates, four gate drivers which provide a high operating voltage isolation are used. The gate drivers are HCPL-3120 Isolated IGBT Gate Drive, ideally suited for motor control inverter applications. This isolated gate driver has been used to protect the controller and low voltage side of the system while providing sufficient drive voltages and current to drive the gate controlled IGBTs. The optocoupler of this driver consists of a LED optically coupled to an integrated circuit with a power output stage. 


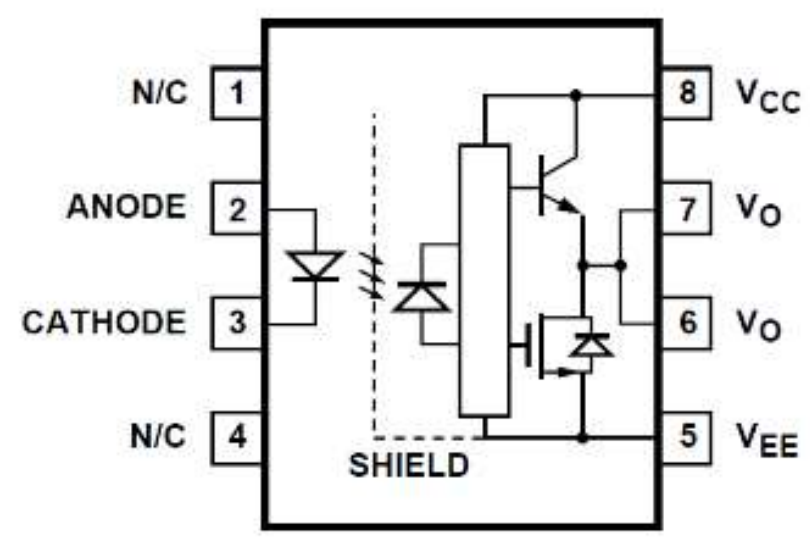

Figure 19. Functional Diagram of the Gate Driver

Since different components of our circuit operate on different voltages, we employed multiple $\mathrm{DC} / \mathrm{DC}$ converters to power the whole circuit from a single $24 \mathrm{~V}$ power supply. We selected two converters with different output ranges, based on the required specifications of the design, the limitations of the main components, and the availability of the DC/DC converters in the market. The VQA-S24-D15 converter was used to supply the gate drivers, which require a 15 volts power supply, and PDM1-S24-S5 to supply the logic side of the circuit by a 5-volt power source. Both of the converters can operate with a 24 volts power supply.

Collectors of the top side of the H_Bridge and emitters of the bottom side of the H_Bridge are connected to the high capacity power supply which provides the required voltage and current to drive the machine. 


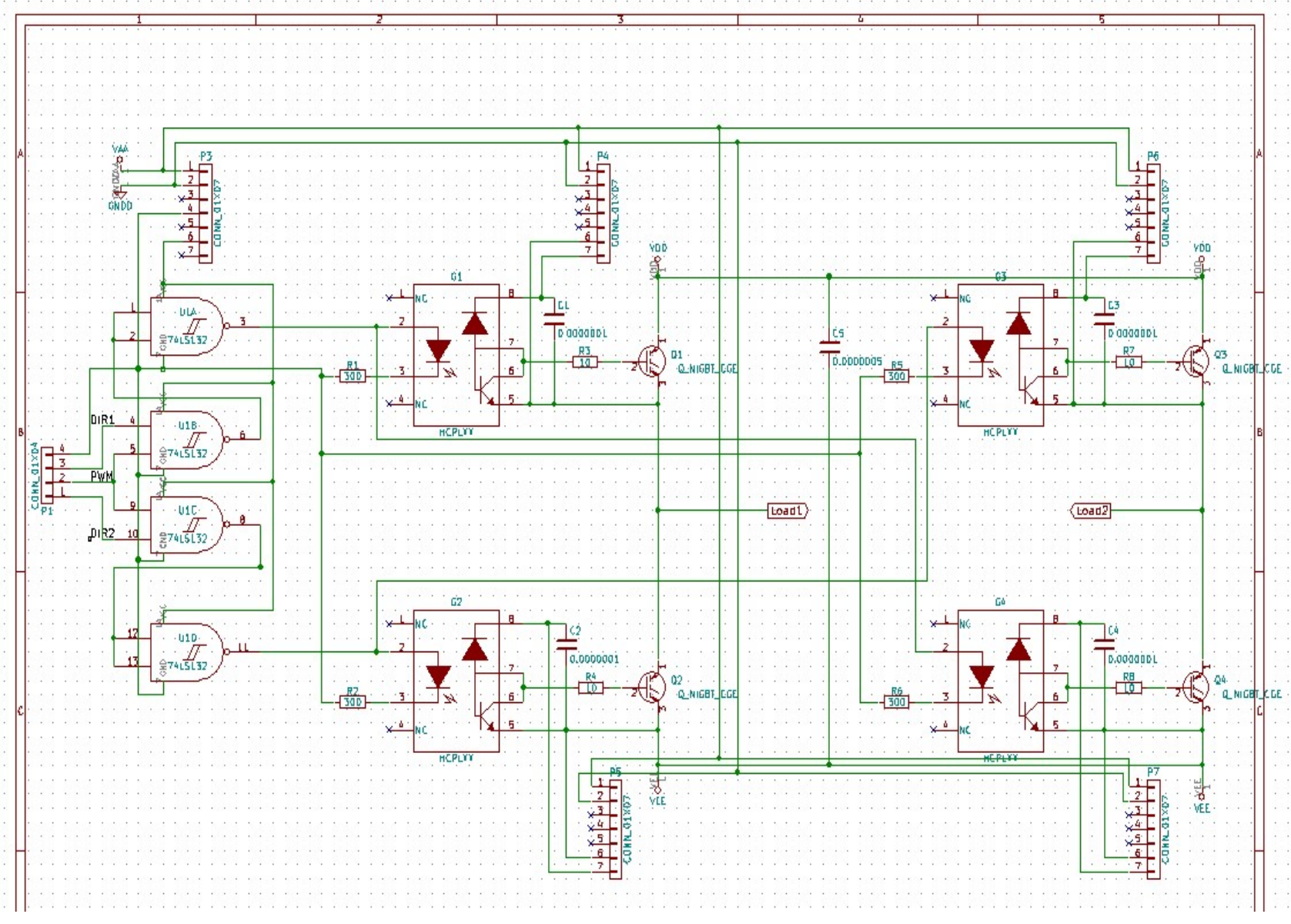

Figure 20. Schematic Design of the Inverter Circuit

The emitter of Q1 (see Figure 20. Schematic Design of the Inverter Circuit) is connected to the Q2 collector and the VEE of the optocouplers to prevent it from floating, while Q2 and Q3 are conducting and Q1 and Q4 are switched off.

The logic configuration of the circuit is providing the gate command signals to switch them on and off and direct the current in the desired direction. The control signals come from the DSP and are sent out to the gate drivers to drive the motor.

The DSP sends three control signals to the inverter, two signals for direction and another one for the PWM. These three signals are fed to a logic NAND gates to build the PWM signal, and transmit two command signals. These command signals activate the switch gates and drive the load. Since at each time two switches, are on or off together at the same time, only two drive signals are needed for the IGBT gate driving. One signal turns on a pair of switches and the second signals turn off 
the other pair of switches, simultaneously. Therefore, the two outputs of the NAND gates which goes to the optocouplers, are complementary logic signals.

The direction signals determine the direction of movement, which indicates which pair of switches is supposed to be turned on or off. When the direction signal state is high, the corresponding switch is connected. On the contrary, the switch is disconnected when the direction signal is low. Note that the direction signals are two complementary logic signals which change the state of the inverter switches.

The PWM signal is a $10-\mathrm{kHz}$ square wave signal with a variable duty cycle. It changes the excitation voltage of the machine, and consequently, alters the speed of the motor. The direction signals at on state should be AND with this signal to change the constant ON state to a duty cycle variable PWM signal. This signal changes the average value of the applied load voltage.

To perform the logic AND, a Quad 2-Input Schmitt Trigger NAND Gate SN54/74LS132 is used, which consists of 4 logic gates in an IC package.

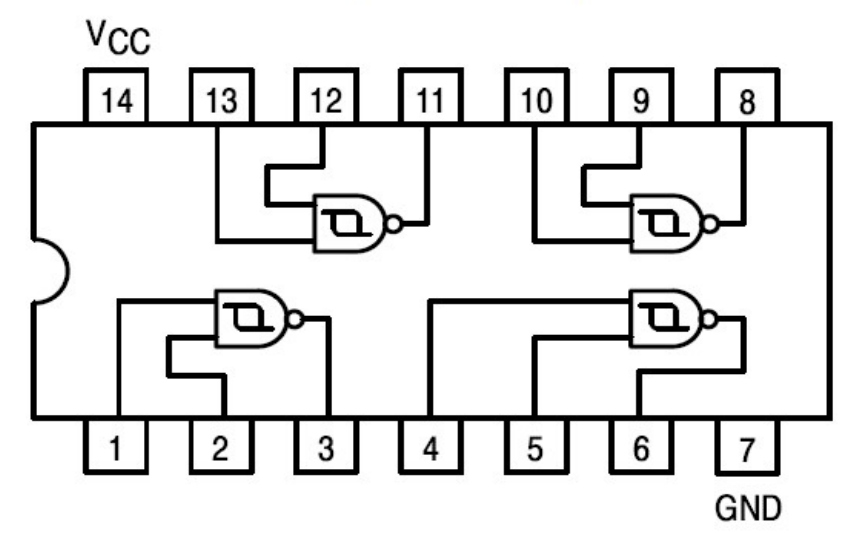

Figure 21. Quad 2-Input Schmitt Trigger NAND Gate

Building a logic AND gate from a logic NAND gate is possible as illustrated in Figure 22. Logic AND Diagram Built by NAND Gates. 

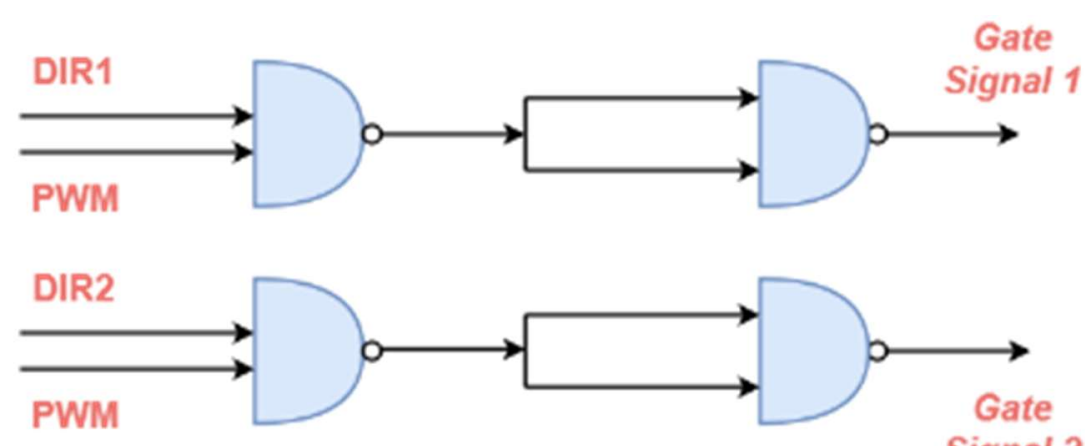

Figure 22. Logic AND Diagram Built by NAND Gates

Note that, the PWM signal is considered in the design, even though to simplify the system, it is replaced by a constant voltage. Then the speed can be controlled by adjusting the power supply voltage as a constant value, not a PWM signal.

\subsection{PCB Design}

The OLEA system environment are noisy with lots of electromagnetic interferences (EMI), therefore, it is necessary for all the noise sensitive electrical components to be on printed circuit boards with a ground plane. KiCad PCB design software was employed to plot the circuit schematics for the design of PCBs. KiCad is an open source software to create Gerber and layout files to print out the PCB board.

The software includes some ready to use component footprints, such as HCPLXY, which has been used to indicate the optocouplers. On the other hand, the missing elements in its library have been drawn manually, based on the pinouts provided in the IC datasheet, such as the IGBTs.

The designed board is in size of 8 by 3.8 inches. A two-layer PCB with 0.062 inches thickness was designed, with approximately 130 holes, and masked on both sides to protect it from unwanted connections. The required safe distance between the traces and components has been considered in the design process. The trace widths have been calculated for the predicted current passing through them, based on the thickness of the board and the current passing through. 


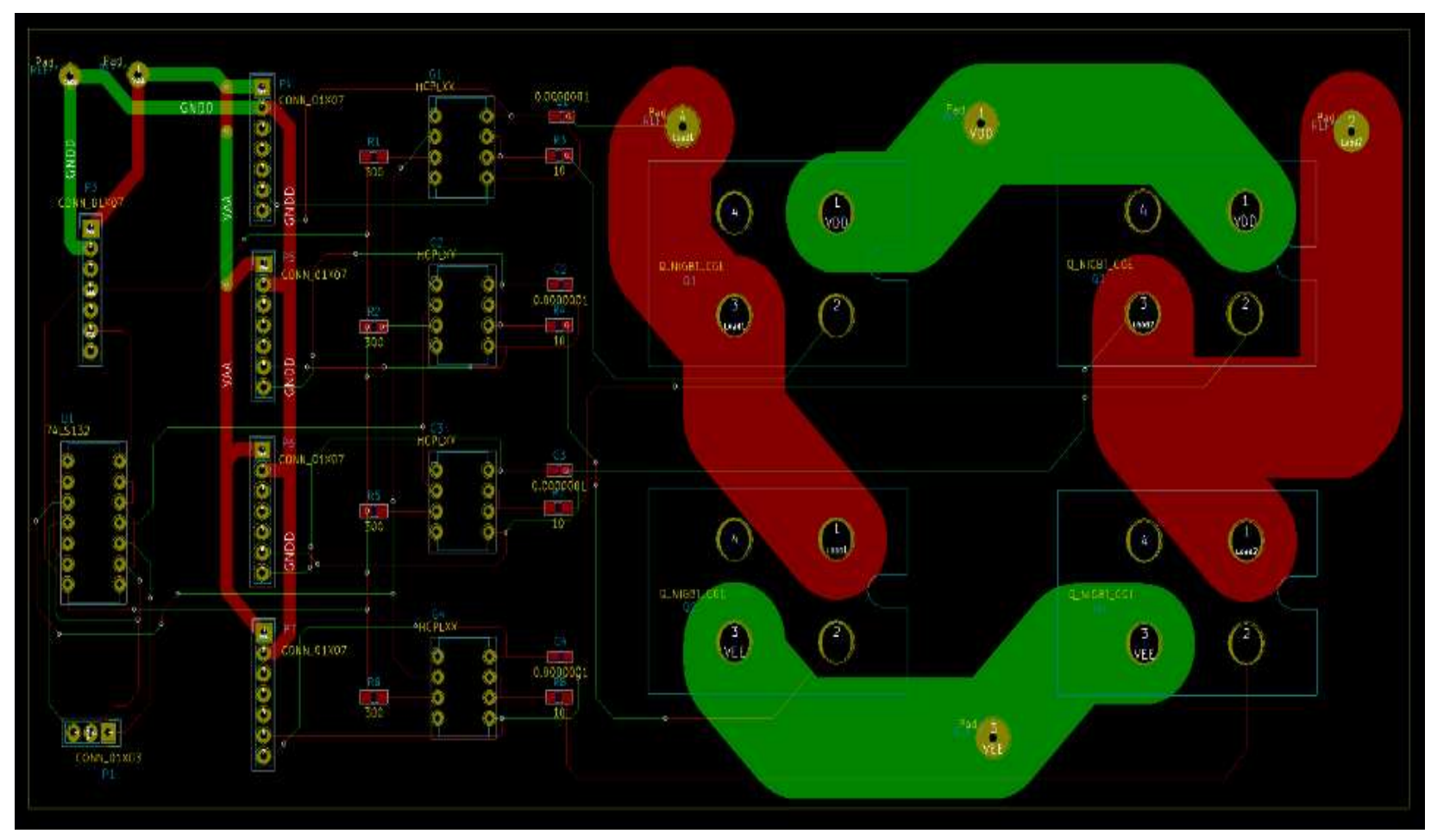

Figure 23. Inverter PCB Design

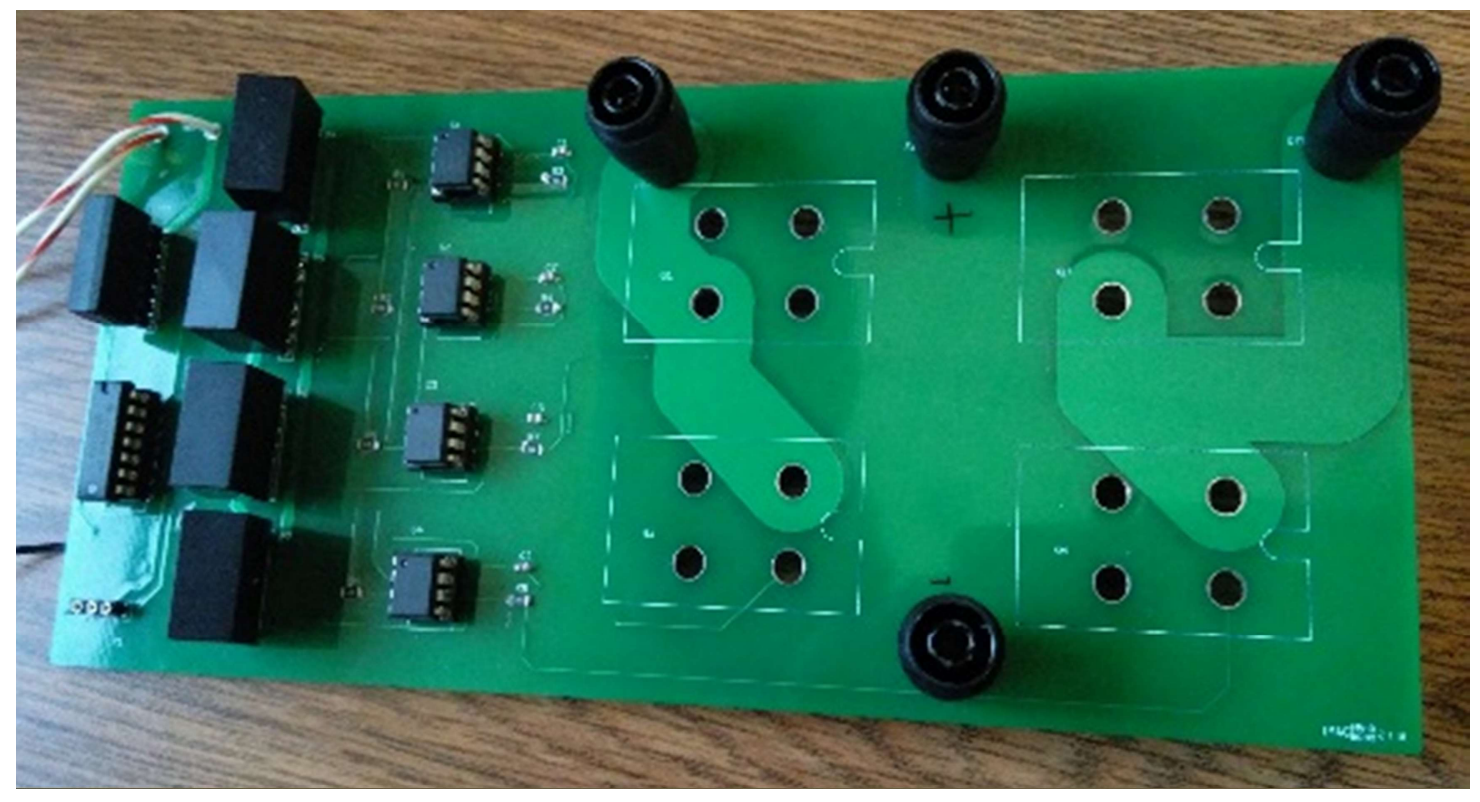

Figure 24. Inverter Board with Components 


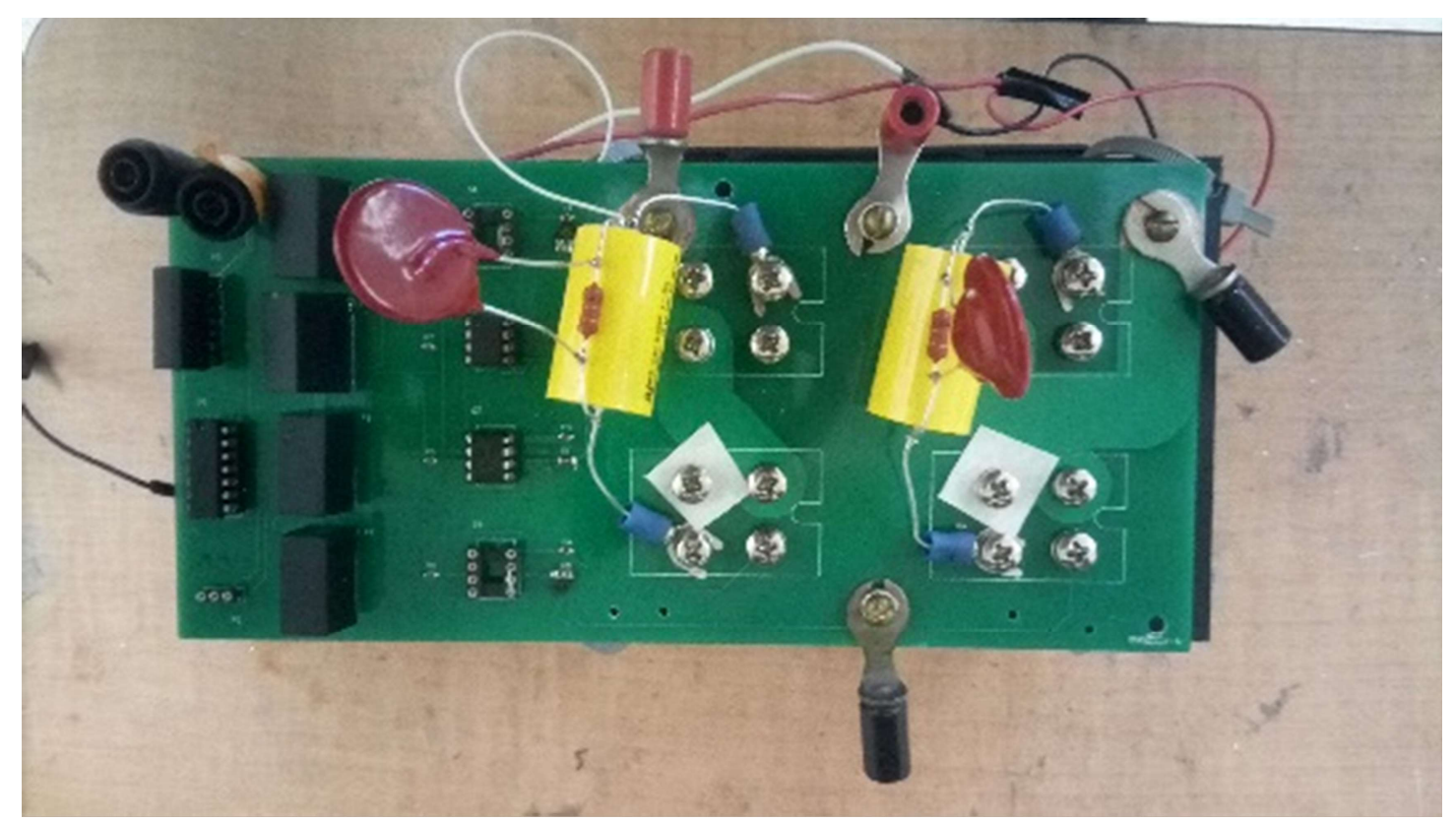

Figure 25. Inverter Board Ready to be Connected to the Machine

The next generation design was upgraded by changing the Surface Mount Devices (SMD) resistors to the through hole packaging, changing the hole radius of the power supply and motor connections connectors, increasing the copper area around the IGBT connections to have larger mating surface, and adding four holes to install four legs on the board to stand it. 


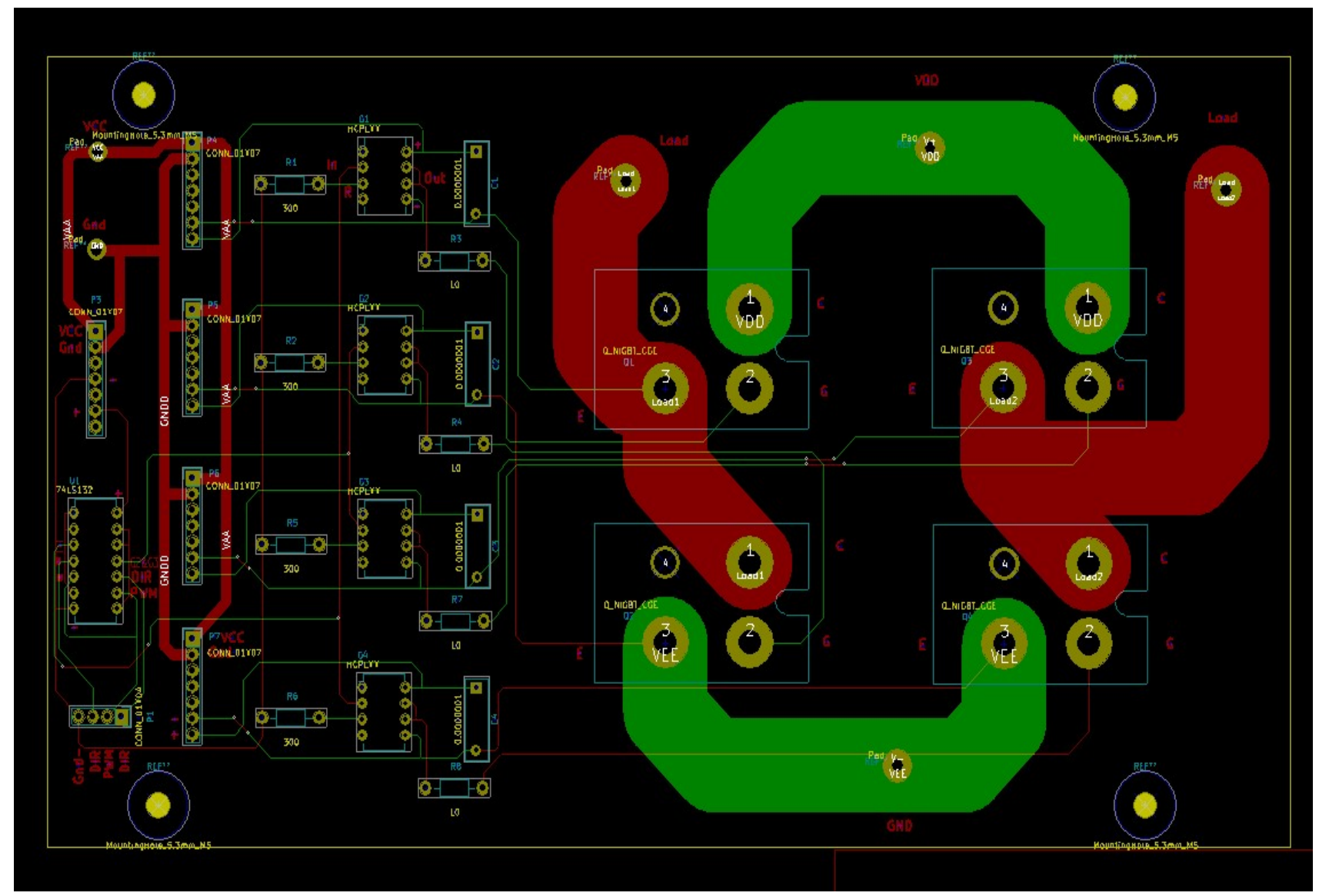

Figure 26. Modified Inverter PCB Design

\subsection{Starting from Standstill}

Since we are using an incremental encoder as the position sensor, the position data is only available when the machine is running. Incremental encoders are only able to provide relative position, and they are not able to detect the starting position. On the contrary, we need the exact position of the translator to generate the driving voltage pulses. Accordingly, the machine needs a starting procedure, referred to as transient mode, before switching into the PLL mode. When the machine is running in its transient mode, the information about the maximum and minimum of the position is available to the controller. Therefore, the controller can switch to the PLL mode to make the output synchronized with the motion waveform.

The controller in its transient mode runs the linear motor for a few seconds with a fixed motion frequency. When the translator movement is stable the controller switches to the PLL control scheme. Running the machine in the transient mode for a few seconds, and in an open loop fashion, 
is sufficient for the linear motor to start running at its resonant frequency. The open loop mode is sending out a constant frequency pulse signal to the machine. At this point, the PLL functions while the machine is resonating near its natural frequency. The position data is also accessible when the PLL control scheme starts operating.

\subsection{Control Strategy in Motoring Mode}

In the OLEA machine, transferring the energy in an efficient way is a crucial task to improve the performance. Current flow in the stator windings must be synchronized to the instantaneous position of the translator. In other words, the stator magnetic field is supposed to be aligned with the permanent magnet field of the alternator. Therefore, the driving pulses of the machine or the phase excitations should be adjusted to the linear motor movement. Running this class of motors is dependent on the translator position information which enables the controller to synchronize the phase winding excitation with the translator position.

Thus, the translator position relative to the stator coils is required to be measured and transmitted to the current controller. The transmitted information, then, can be utilized to commutate the current in the stator windings, and to calculate the control input precisely for optimum torque production. For this purpose, usually, the position information is measured by different types of position sensors. Then, the optimum control can be achieved by communicating the position data to the controller. The translator position information is also required for speed control. The input voltage that drives the motor should be under the control for speed control.

This requirement implies that the translator position needs to be either sensed and measured, or estimated. The control procedure is manipulated in a closed loop mode and the position data is being fed back to the controller. Consequently, the performance significantly improves by this closed loop negative feedback system. The controller electronically functions based on the realtime position data provided by sensors, or following a sensorless approach in single phase [36], [37] and in three phase [38]-[44]. To achieve this goal, there are many different control strategies

which can perform in a sensor or sensorless mode. In the latter case, different types of sensors can be used to measure the position of the rotor, such as Hall effect sensors, Optical sensors, Inductive sensors, or Incremental Encoders for high accuracy position detection. 
The microprocessor or the DSP, which is functioning as a controller, feeds the driving voltage to the motor through an inverter. Note that the inverter's frequency is a function of instantaneous translator speed and its phase is adjusted by the actual translator position [45]. Accordingly, the DSP combined with the inverter develops a motor control unit whose input data comes from the translator position.

The system in this development is a Linear Tubular Brushless Permanent Magnet Machine with a sinusoidal flux distribution that is controlled by a Phase Locked Loop technique. The translator position information is supplied to the controller through a position sensor. Even though continuous position detection is not required, this system is able to detect the position with high precision in real time. The controller detects the zero crossings of the speed waveform which are the peaks of the position signal. This detection is required to synchronize the current through the inverter. It, then, generates a driving pulse to excite the motor phases and drive the machine. The pulses define the conducting period of each phase windings. The inverter changes the direction of conducting current in the windings to make reciprocating movement in the alternator, and supply the required voltage and current to run the machine. The proper timing of the gate signals is a significant issue in driving the machine which is explained in the following section.

\subsection{Gate Signal Timing}

In this mass-spring oscillatory system, the position signal is a sine wave. Driving force is in the same direction as the translator's motion to get the maximum displacement. If the controller generates the driving pulse in a wrong direction, against the direction of translator movement, it pushes it in the opposite direction and is counteractive. Therefore, the excitation pulse should be accurately synchronized with the movement and be in the same phase and direction.

The direction of the mechanical movement changes at each peak position. The spring force direction remains the same at every quarter of a cycle and it changes between every two subsequent peaks. To input the energy into the system and have a positive energy, force and displacement should be at the same direction. Accordingly, the best two points for motor excitation voltage pulse generations are the maximum and the minimum points of the position sine wave. The DSP and the

inverter drives a current into the motor phases that makes the machine moves in the direction of the spring's force. 
These two points are allocated to be the start point of the pulsing. The pulse state should be high during the second and fourth quarters of the position sine wave. During the running time of the motor, the controller detects these two points and generates a pulse at each point to turn ON inverter gate drivers in sequence. Each pulse has a predefined pulse width, less than a fifty percent duty cycle. From one peak to another, the motor is moving forward, then it stops and returns in the backward direction. At each peak, the translator speed is zero and the machine changes the direction of its movement.

In a concise manner, the controller is supposed to detect the peaks and sends out a pulse with a width of 25 percent of the duty cycle to keep it within the same direction as the motion. The driving pulses provide the energy to move the translator while the spring forces push it back.

A pulse needs to be sent to the inverter to switch on appropriate semiconductor devices so that the current flows throughout the path. The pulse should be at a specific point according to the position signal. The desired control system is needed to ensure that the gate signal is fixed at its location. PLL guarantees that the driving gate signal is in phase with the motion signal.

\subsection{Phase Locked Loop Control System}

\subsubsection{A Simple Description of the Mechanism of a Phase Locked Loop}

Generally, a Phase Locked Loop is a closed loop negative feedback control mechanism which is forcing the error signal to approach zero.

A negative feedback control loop system consists of these main elements: reference signal, system output, measured error signal, sensor in the feedback path, measured feedback signal, controller, system input, and the plant. 


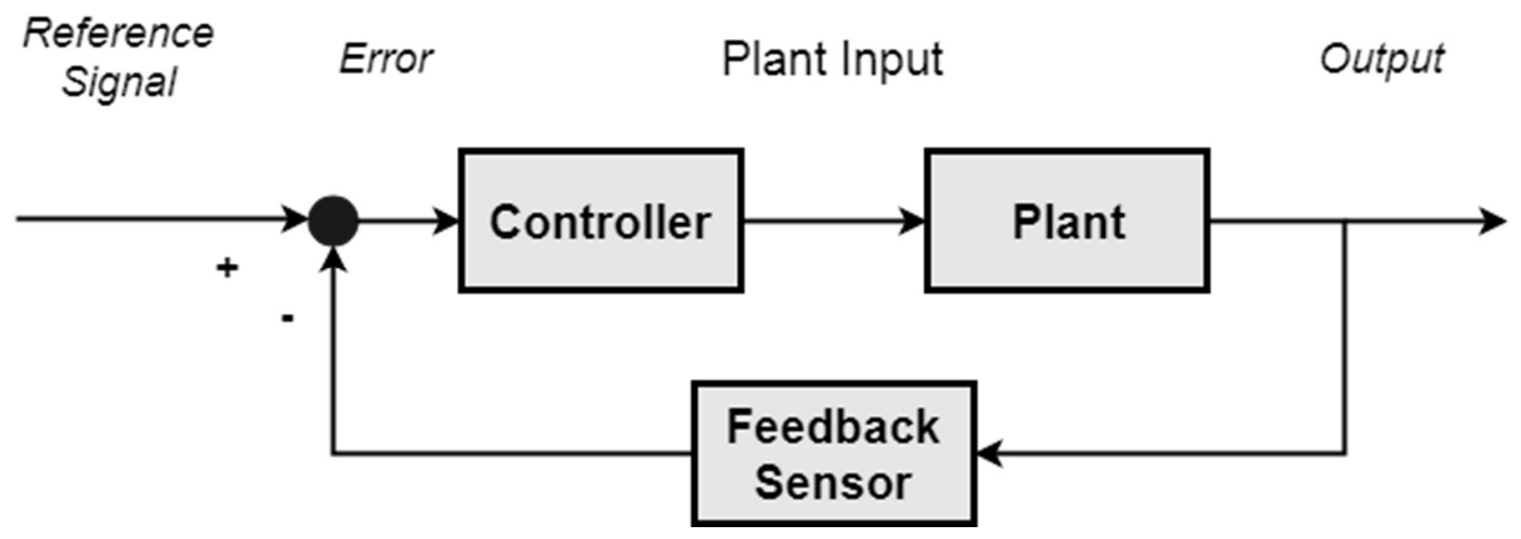

Figure 27. A Negative Feedback Control System Block Diagram

There are two different practical types of PLL, namely digital and analog PLLs. While analog PLLs are driven by an analog circuitry, a digital PLL can be driven digitally either by the means of a digital to analog converter, or pure digital elements. The nonlinear procedures in both types are the same, while the former is built using digital elements, and the latter is built upon analog elements. A basic PLL is an electronic circuit including some basic nonlinear components, such as a variable frequency oscillator and a phase detector [43]. Variable frequency oscillator is a controller whose output's oscillation is adjustable by a low-frequency input voltage [44]. A phase detector, or phase comparator, is a sensor to measure the phase difference between the reference signal and the feedback signal from the output.

The desired value for the phase difference is a constant or zero. The phase detector compares the signal generated by the oscillator, which is the negative feedback, with the periodic oscillating reference signal to acquire the phase difference relative to the reference. Then, the phase comparator sends out the error signal to the oscillator, which is supposed to approach to zero, in order to compensate for the phase difference. The error signal is a voltage that has been generated by the phase comparator according to the phase difference. This error is then utilized to control the oscillator output signal. Finally, the oscillator matches the phase, based on the error signal, and consequently, the output frequency follows the input signal frequency. This combination, that generates the required system input signal, encourages the frequency and phase to follow the reference. 


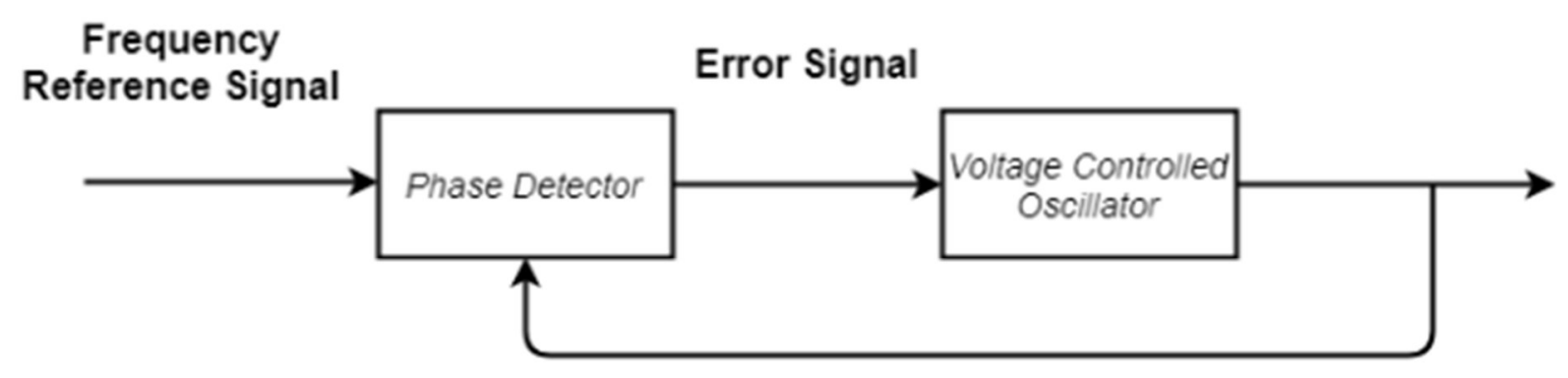

Feedback Signal

Figure 28. PLL Configuration

Since the phase detectors are able to adjust the output frequency to the reference, for the sake of simplicity, it can be assumed that the frequency of the oscillator is initially close to the frequency of the reference signal. However, in practice, oscillators are typically tuned to the desired frequency.

An oscillator generates a signal with a frequency close to the reference frequency. However, it is not synchronized with the reference signal. To synchronize them, the oscillator signal is fed to the phase detector through a negative feedback. Therefore, the phase detector compares the reference signal and the oscillator signal to detect the phase difference and generates a voltage proportional to the phase difference. Then, this error signal drives the oscillator to make a phase shift in the output. The shifted output goes again through the negative feedback and input to the phase detector. If the output signal phase leads ahead of the reference, the error signal forces the oscillator to slow down and reduces the phase difference. On the contrary, when the output falls behind the reference, the error forces the oscillator voltage to be faster by increasing the frequency to compensate for the delay. Thus, the error drives the oscillator to move in an opposite direction relative to the output. Based on the difference between the reference and the output, the phase detector increases or decreases the output control voltage of the oscillator, which is the error signal. After it locked onto the reference, the error signal is in a steady-state mode.

\subsubsection{PLL Applications}

There are several variations of PLLs in electronic circuitry which are specialized in hardware or software. Hardware designs, in both analog and digital sense, often utilize XOR gates to implement 
a simple phase detector in a PLL circuit design. On the other hand, software PLLs are implemented through the use of functional blocks by software.

PLLs are commonly manufactured as integrated circuits (IC), and also as discrete circuits to be applicable in analog transmission and microwave signal processing as well.

Since the PLL technique is developed and manufactured in a single IC, it is widely used in modern electronic devices. The off-the-shelf ICs can function as a complete PLL block, and provide outputs with a wide range of frequencies, from a fraction of a Hertz to many Gigahertz.

PLLs are being widely used in communications [46], such as recovery of a signal which is lost in the noise from a noisy communication channel by tracking the reference signal, and also in digital data transmission. It can also be employed for synchronization purposes in telecommunication, space communication, and wireless communication for frequency modulation (FM) or phase modulation (PM) transmissions. Commonly, the PLL applied in communication systems and radio to stabilize, modulate/demodulate and filter the data when it has been interrupted.

PLL has been also employed in electronics and computer applications, primarily in digital logic circuits and microprocessors for computer clock synchronizations. For instance, in a microprocessor, the internal processor elements need to run faster than the external connections while the timed clock pulse and the timing relationships are supposed to be distributed precisely. For this purpose, PLL has been utilized in clock multipliers. The clock timing information can also be recovered from a data stream, e.g., like a disk drive, by the means of a PLL.

In the power electronic industry, PLL has been used for grid tie inverters, like renewable energy sources, and in PMBLDC motor drivers, as variable speed drives, in a wide variety of applications for their benefits [47] which will be described in detail in the followings.

The PLL technique has been vastly studied for precise speed control and regulation, and fast dynamic response [48], [47], [49], [50], and [51]. On the other hand [5] focused on improving the operation efficiency in modern industrial applications by utilizing a PLL to adjust the commutation angle of the inverter switch. The winding current lags the back-EMF voltage because the PM motor represents-is an inductive load. Therefore, the lag current generates more reactive power, which results in efficiency degradation of the motor. It can also cause extra vibration and acoustic noise $[5]$. 


\subsubsection{PLL in Control Systems}

Phase locked loop is a control framework that its output signal yields from its input signal. More specifically, the dependent output of the PLL is constantly adjusting its phase, and consequently its frequency, to follow the phase and frequency of the input signal. In other words, PLL keeps the phase and frequency of the output locked onto the input signal and synchronizes the oscillating voltage or current signals. Basically, PLL generates a voltage signal which tracks the variable frequency input signal by comparing the phase of the output voltage signal to a reference input signal. It can produce a stable high-frequency output at a multiple of the input frequency, which is a fixed low-frequency signal. This process is called frequency synthesis and is useful in communication applications.

Generally, the phase difference between the two signals of different frequencies always varies. In contrast, when their frequencies are the same, the phase difference remains constant at all time. Therefore, a constant value phase difference implies that the frequencies are the same, and the output is either leading or lagging behind the input by a fixed amount of degree or time. Since the phase is proportional to the time, in the time domain, a difference in phase can be also interpreted as a certain amount of difference in time. The phase difference is the measured angle between certain points in input and output waveforms. Note that these points can be selected at any point, such as the zero crossing point.

\subsubsection{PLL Implementation}

In this system, the motor input voltage is required to be synchronized to the oscillating motion signal to achieve maximum translator displacement. Therefore, implementing a PLL control is a suitable method to achieve the goal with a high accuracy speed regulation, which is provided by a current loop control [47].

The main goal of this control system is to keep the drive voltage pulses in phase with the position signal. To have the maximum displacement, the pulse must occur at the maximum or minimum point of the position signal, where the speed is zero and the translator is at one of the ends of the strokes. 
This approach, for implementation of a software-based PLL in the linear motor, is acquiring position data by a sensor and producing the output signal by a programmable DSP locked onto the position signal. The controller outputs are pulses which drive the motor. PLL runs the motor while synchronizing it to the motion signal.

Software-based PLL is more reliable and durable since it does not need any maintenance. In addition, it is more flexible and enables customizable functions based on the system requirements. The most critical situation that hardware-based PLLs cannot handle is that both amplitude and frequency are changing during the running time of the machine. The hardware-based PLL techniques cannot decide based on the frequency or the position peak value as they vary by time. Consequently, the available PLL chips on the market do not meet our requirements.

The software-based PLL control techniques are entirely implemented by code, running inside a DSP. The only hardware elements that are required in our design are a position sensor and a DSP.

The input to the control system (DSP) is the sinusoidal position waveform information coming from the position sensor. The outputs of the control system (DSP), which are the excitation pulses, are supposed to be kept at either minimum or maximum of the position waveform at each cycle to keep it in phase with the position. Consequently, the phase difference between the position and voltage pulses remains zero at all times. The DSP processes the input information and waits for the desired moment while monitoring the position signal. It determines the instant to generate the output pulse to synchronize the input voltage to the motion.

\subsubsection{Secondary PLL Applications in Our System}

In this system, PLL is also responsible for determining the resonant frequency. Trying different frequencies to find out which one maximizes the translator displacement is a tedious task. Finding the exact resonance frequency is not also a simple task by trial and error. However, the PLL provides a quick and simple way to measure the accurate resonance frequency. Note that when the machine is running in the PLL mode, there is no control over the frequency, and it cannot be set by the user. In this state, the system oscillates at its own resonance frequency. The accuracy of this process has been checked by hitting the translator's shaft by a mallet that makes the moving part 
oscillates for a few seconds at its resonance frequency and by capturing the position signal on the oscilloscope. Following this approach, the true resonance frequency of the motor can be found.

Moreover, the springs in the machine are nonlinear and their stiffness varies with displacements. Consequently, the operation frequency changes according to the spring's stiffness. The range of this frequency is small, however, it is not totally fixed. This variable frequency situation can be addressed by utilization of PLL. A PLL-based control system, which synchronizes the ignition pulses with the translator position, can adjust the control input to the frequency changes.

Utilizing this method, output control signals, i.e., ignition pulses, are generated. As it is mentioned before, the ignition pulses must occur at two position extrema, and be adjustable in a predefined range.

However, it is not practical to run the electric machine when in its motoring mode on PLL control directly. In fact, it requires a starting procedure, then the controller can switch to PLL mode and run the motor. Then the engine starts, and the controller switches to generating mode. In this mode, the controller generates the ignition pulses to keep the engine running at the motor resonance frequency.

Theoretically, the PLL signals can be generated at either one direction or both moving back and forth. However, there are a few considerations in this system which will be discussed.

\subsection{Single Pulse and Pair Pulse}

Having two direction signals enables the controller to energize the motor with a single pulse in one direction, or with a pair of pulses in both directions. By clearing the second direction pin, i.e. keeping it in the low state continuously, the direction is controllable by only one direction signal. The controller, then, needs to send a single driving pulse. In this case, only one pair of inverter switches is ON during the whole period of running the motor, and the second pair is off. However, driving the machine with a pair of pulses is not efficient since the springs can push the translator back, while a single pulse is being used to run the motor in a fixed direction. 
Moreover, the machine is pulsed in an opposite direction of the cylinder head since the piston should not hit the cylinder. The pulse is moving the piston far from the cylinder not to move it toward the cylinder head. This condition is to avoid colliding the piston and cylinder head.

However, running the motor with either a single pulse or a paired-pulse is associated with an important issue. This issue arises when the orientation of the polarity of the current flows through the machine is not in the same direction as the movement. More specifically, when the controller intends to move the machine from left to right, it switches ON one pair of IGBTs to flow the current. The current creates a thrust force to move the machine from left to right. If the current flow polarity is not in the same direction as the movement, the voltage opposes the movement, and the machine will not be able to oscillate. In other words, if the machine is at the right end, then the current flow direction is not able to push it from left to the right. Therefore, the current polarity should be reversed to move the translator from right to left. The current polarity and thrust force must be synchronized to the motion direction and should remain the same while the translator is moving in the same direction.

\subsection{Dead Band Consideration}

An important consideration in designing an inverter is to prevent the "shoot-through" occurrence. Shoot-through is a serious short-circuit condition which occurs when the high voltage side of the inverter turns ON before the low voltage side turns OFF. This can cause a short circuit across the voltage source to ground. That is, both the high and low voltage side IGBTs are on at the same time. Shoot-through can happen when one side is commanded to turn on and the other side is commanded to turn off. The IGBT turn-on delay and turn off delay times can cause a short period when both sides are on. In that case, current flows directly from the voltage source to ground through both IGBTs. Therefore, a mechanism is needed to block this short circuit.

In this design, it has been taken care of by the software. In other words, the DSP signals the gate drivers when only one pair of switches is on at every moment. During running the machine by PLL or open-loop with a single pulse, it is clear that only one set of IGBTs are turning ON and OFF. However, when two complementary square waves or a pair of pulses are being used to drive the machine, it is not guaranteed that only one set of IGBTs turns on. Therefore, only these two 
cases need to be dealt with, namely a pair of pulses and two complementary square waves. This case can be handled by the means of dead band timings.

Even though running the linear motor with a pair of pulses does not need any special consideration, since one pulse is in the forward movement and the other one is in the backward movement, when the duty cycle of the pulses increase to fifty percent, shoot through is likely to happen. A pair of pulses with fifty percent duty cycle can be considered as two complementary square waves.

To overcome the shoot through the problem, the built-in PWM module of the DSP was used to create a dead band for the IGBTs. Consequently, an appropriate dead band of $280 \mathrm{~ns}$ has been selected, based on the datasheet. Hence, by making a small delay or lag in turning on and off the signals, in a way that they do not overlap, the shoot through problem can be effectively prevented.

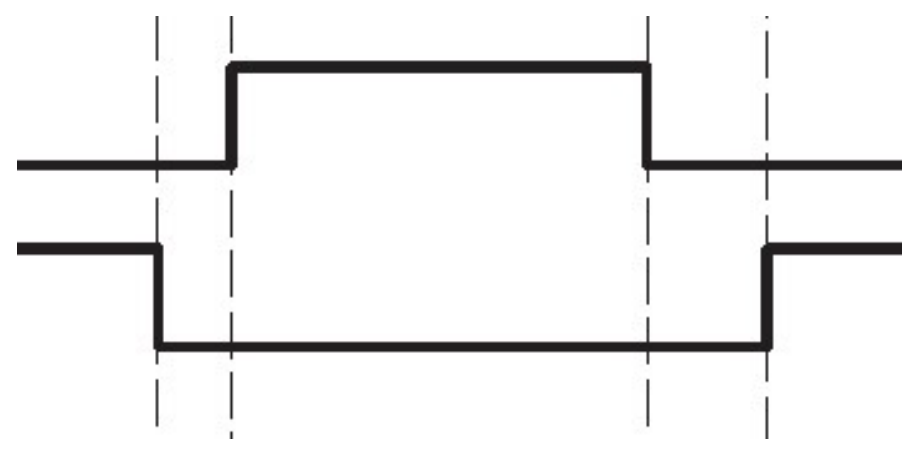

Figure 29. DSP Dead Band Handling

However, in the case of using a pair of pulses to run the motor, we do not need any dead band consideration, as long as they are with a duty cycle of less than fifty percent. 


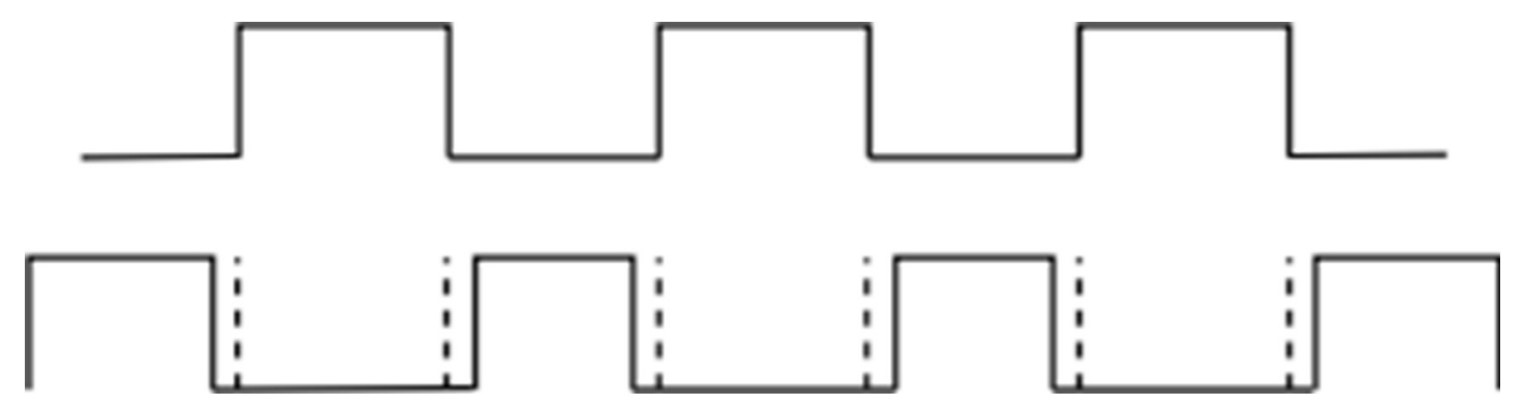

Figure 30. Complementary Square Waves

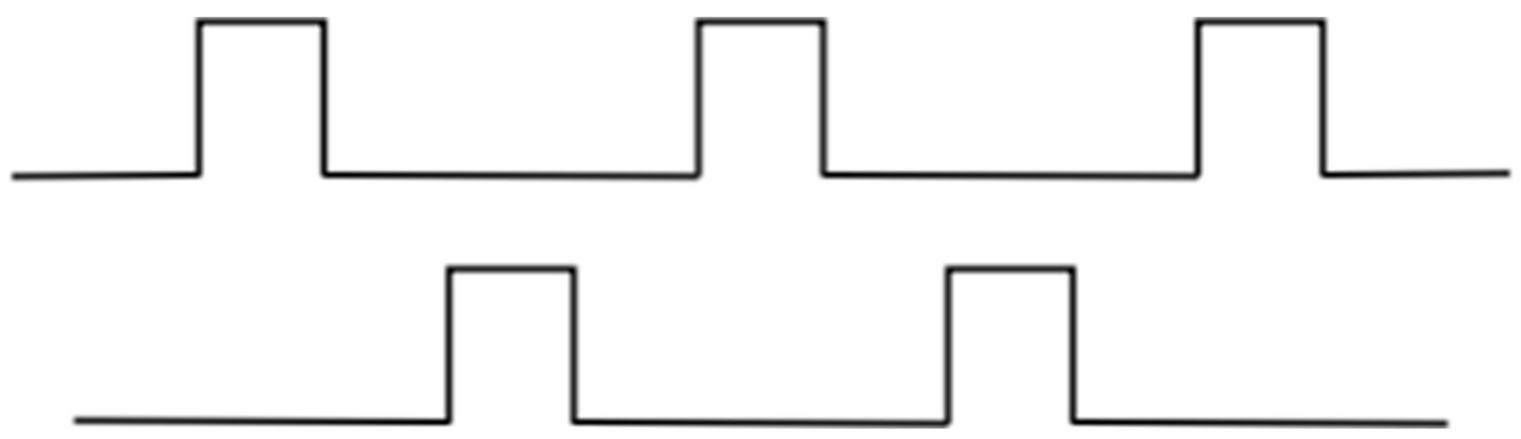

Figure 31. Pair of Pulses

\section{OLEA Operation}

\subsection{Control Strategy in Generating Mode}

To run OLEA as a generator, the coil ignition and fuel injection are essential to start and run the engine and make combustion to occur.

The flammable range for ignition is 10 percent of a cycle before or after the Top Dead Center (TDC). The TDC of the cylinder is the point in a cycle where the pressure is at its maximum. In this system, it is the peak of the position waveform when the displacement of the linear machine is equal to the stroke length of the engine. If the displacement is lower than the stroke length is lower than the stroke length of the engine, the pressure will be lower than the required compression value and the combustion might not occur. There the ignition pulse should happen near to the TDC to produce maximum output power [52]. 


\subsection{Ignition Signals}

Ignition is a crucial parameter in the engine operation. Therefore, a mechanism to generate ignition signals at the correct ignition timing based on the translator position is required. The ignition system consists of a control system or ignition signal generator, $12 \mathrm{~V}$ supply, ignition coil, and spark plug.

A control signal is required by the controller to ignite the coil. This signal, should be generated at/near the peak position of the translator, to control and run the engine. More specifically, the controller generates the ignition control signal based on the position information provided by the linear encoder sensors.

This ignition control signal is a single pulse with the same frequency as the translator motion. The ignition pulses are located close to the peak position which is consistent with the TDC.

To set the ignition location in a cycle and get the maximum power output, the ignition timing should be set properly. To set the timing, it is necessary to make it adjustable in a way that we have control over the phase of the pulse. Then, we can set it to the optimum phase during the running of the machine. Hence, the adjustable ignition pulse occurs at the point that generates the most output power.

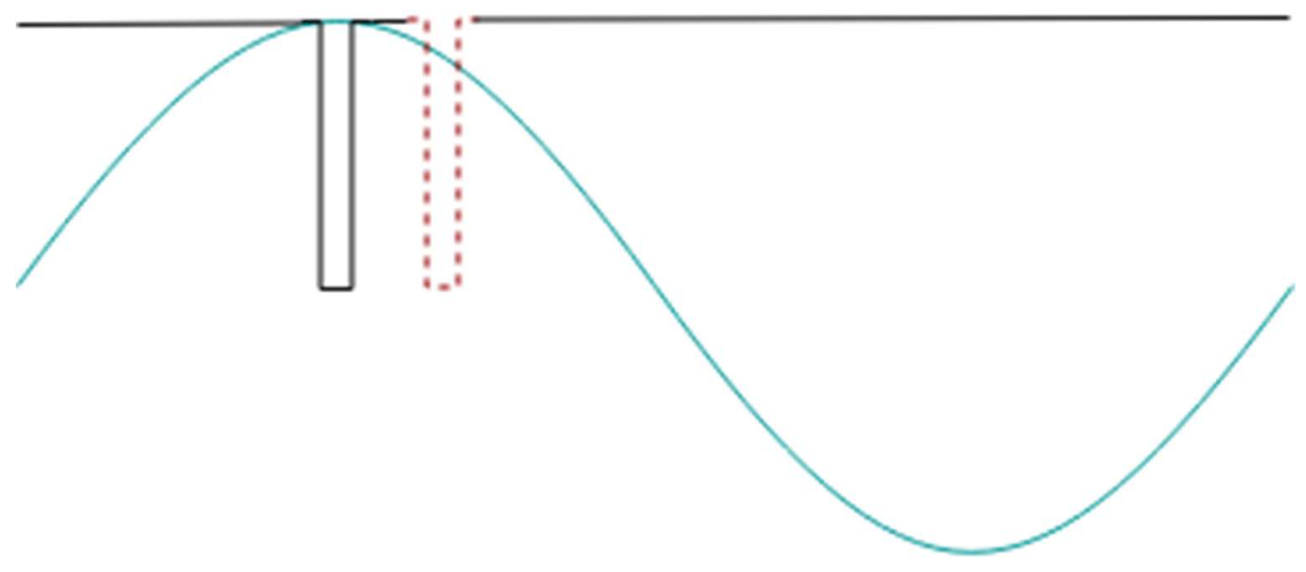

Figure 32. The Ignition Pulse and Position Signal 
For the spark plug to ignite, the time when the ignition pulse occurs is important in every cycle. Pressure is one of the key factors in the determination of ignition timing. Two ignition pulse signals with 2 Milliseconds duty cycle are required in the case of two back to back engines.

The maximum alternator position is the TDC of the first engines, and its minimum is the TDC of the other one. Therefore, the controller generates two adjustable ignition pulses at two position extrema.

Each ignition pulse is an input to its driver board to supply the required voltage and current to ignite the coil. The ignition phase or timing can be tuned by the means of two knobs. The knobs are variable potentiometers which are connected to the DSP as ADC inputs. By turning the knob, a time delay or phase shift is created from the start point of the ignition pulse which is the maximum or minimum position.

\subsection{Control Algorithm}

The control algorithm is implemented in C language and flashed into the DSP using CCS.

The entire algorithm is working in an interrupt-based fashion. The Main function is only responsible to do the Initializations of defined variables, built-in modules, fundamentally involved hardware, and software predefined states, timers, and interrupts. Only Watchdog Timer is running continuously in the Main function unlimitedly to prevent the DSP to restart itself. The main control tasks are implemented inside the timer and other software-based or hardware-based interrupts.

The control algorithm consists of the following steps:

- The encoder provides three signals, namely A, B, and Z, to the DSP based on the movement of the translator. The built-in eQEP module of the DSP counts the number of A-B cycles. The $Z$ signal, however, triggers an interrupt.

- In parallel to the first step, the internal timer module of the DSP is set to trigger interrupt every 300 microseconds. Inside the timer interrupt service routine, the DSP performs the following commands:

- The DSP calculates the derivation of the position signal, i.e. alternator speed, based on the current encoder data, and the history of the position. 
O Using the latest measured speed and its previous values, the DSP check if the speed crossed the zero. Zero crossing detection is required to find the maximum and minimum of the position. The extrema of the position should be calculated using speed zero crossing detection since the displacement of the translator might vary by time.

- The position data is sent to an external DAC through SPI communication protocol. 


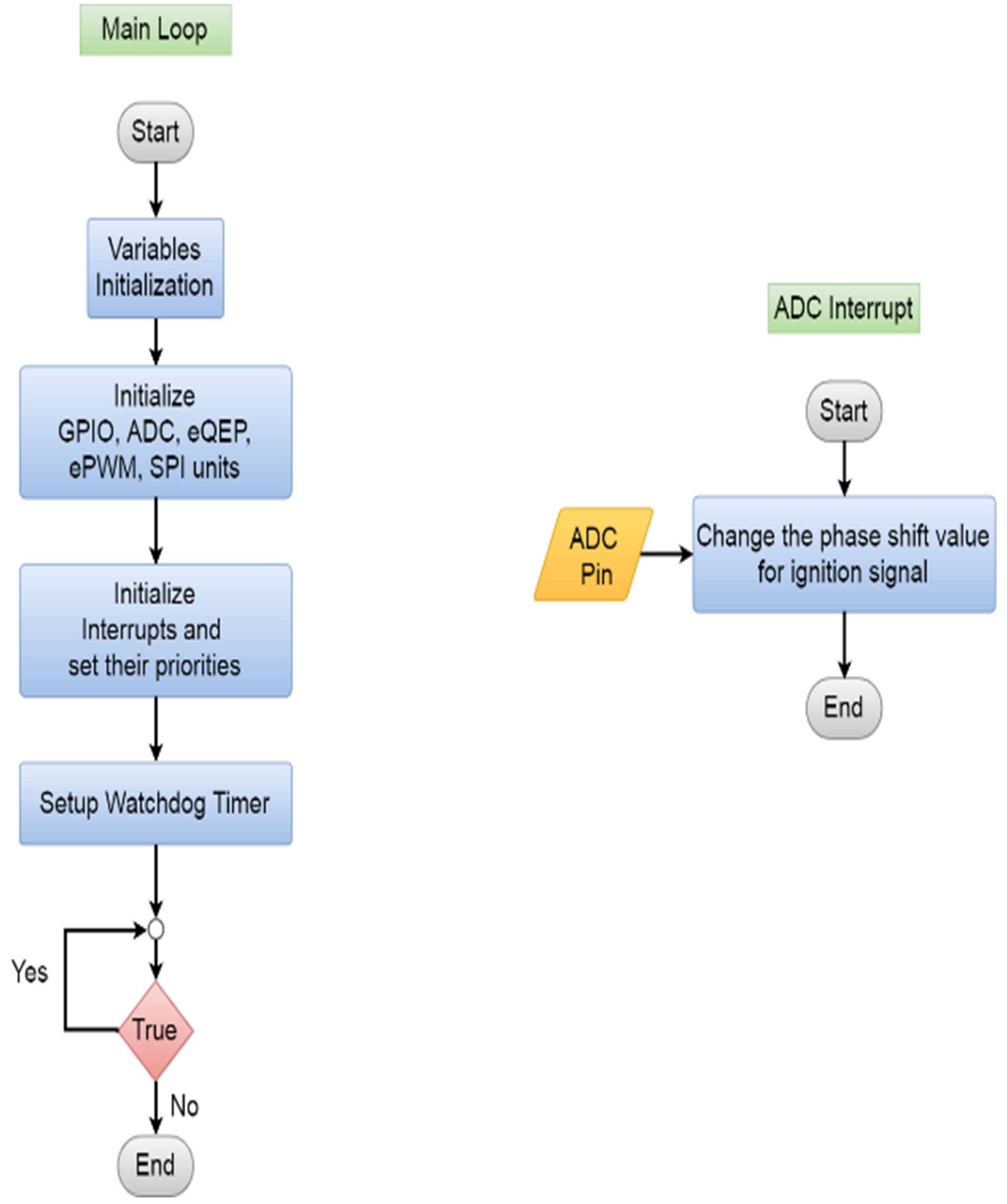

Figure 33. Control Algorithm Flow Chart - Part1 


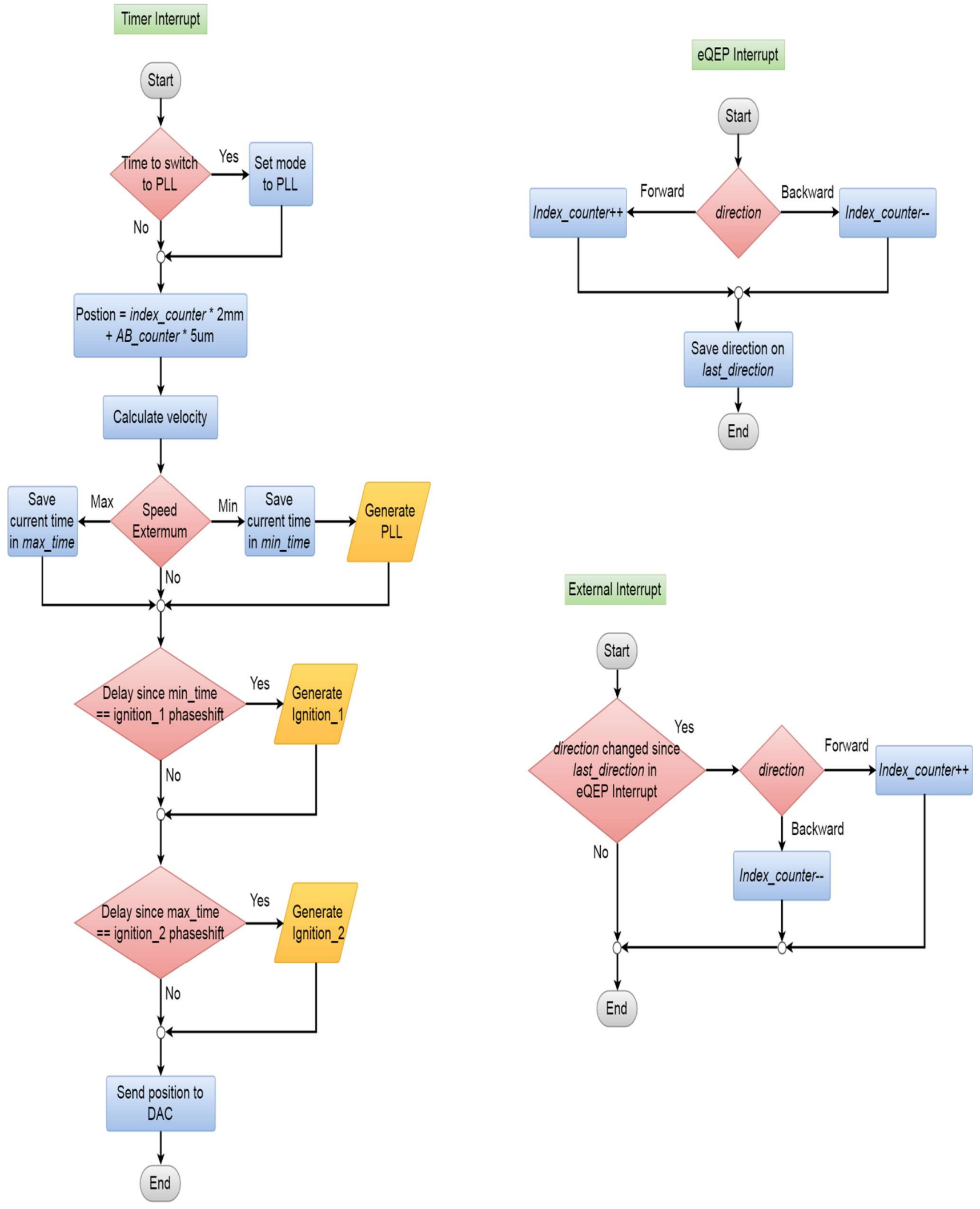

Figure 34. Control Algorithm Flow Chart - Part2 


\subsubsection{Main Function}

Initializations besides the variables include General Purpose Input/Output (GPIO), Analog to Digital Converter module, Enhanced Quadrature Encoder Pulse (eQEP) module, Enhanced Pulse Width Modulator (ePWM) module, SPI module, Timer, and External Interrupt. Mostly, in the Main function initializations must be built and Watchdog Timer should be set up.

The initializations of the different modules equipped in our system within the main function include:

- GPIO: Input / Output pins for the PWM, ADC pins, eQEP input pins, external interrupt, and SPI input have been defined.

- PWM Module: The PWM module requires to be initialized to our needs to start the machine from a stationary state.

- Timer: In timer initialization, we should specify the CPU frequency which is $150 \mathrm{MHz}$, and the timer period that is selected to be $300 \mu$ s. The timer module needs to be started by code at the Main function.

- ADC Module: The registers are initialized and the frequency of conversion was defined.

- eQEP Module: Within the eQEP initialization function, besides enabling the module, the quadrature mode should be selected for our application. The minimum and maximum of the eQEP or AB counter should be declared and the resetting mechanism of the $\mathrm{AB}$ counter is set to be at every $\mathrm{Z}$ interrupt.

- Interrupts: Interrupts priorities need to be assigned based on the code functions.

- SPI Communication: The initialization process declares the DSP as a communication master, sets the number of character bits which is going to be sent as a package, and the clock polarity and frequency.

Watchdog Timer detects the events that the CPU crashes and the program leaves its designated sequence of execution. Watchdog Timer is a free running counter unit, independent of CPU that needs to be cleared periodically by a specific instruction sequence in a specified time period, otherwise, it triggers a reset. CPU must write correct data key sequence to reset the counter before overflow to service the Watchdog or disable it and if the counter overflows, a reset or interrupt 
which the user has chosen is triggered. This must be done within $4.37 \mathrm{~ms}$ after reset that translates into 645000 instructions, while CPU runs at 150MHz.

To prevent the counter from overflow, the valid key sequence should be assigned to the Watchdog Key Register. Note that Watchdog should be serviced in both Main function and the Interrupt Service Routines (ISR) to catch all crashes.

By writing the required values to the Watchdog Timer Control Register, enable or disable the Watchdog Timer, configure the watchdog counter clock rate, and immediately trigger the Watchdog reset can be performed.

\subsubsection{PWM Module}

As a startup procedure, a PWM signal is needed to run the machine prior to switching to the PLL mode. The module generates a pulse with a fixed frequency close to the resonant frequency with a fixed 25 percent duty cycle to run the motor in open loop mode for a few seconds. After a userdefined amount of time, the PWM module is disabled and its output pins act as the output pins of the PLL. Now, the DSP generates the PLL pulses and enters the closed loop control operation. Likewise, the two ignition pulses on the other output pins is activated at the same moment.

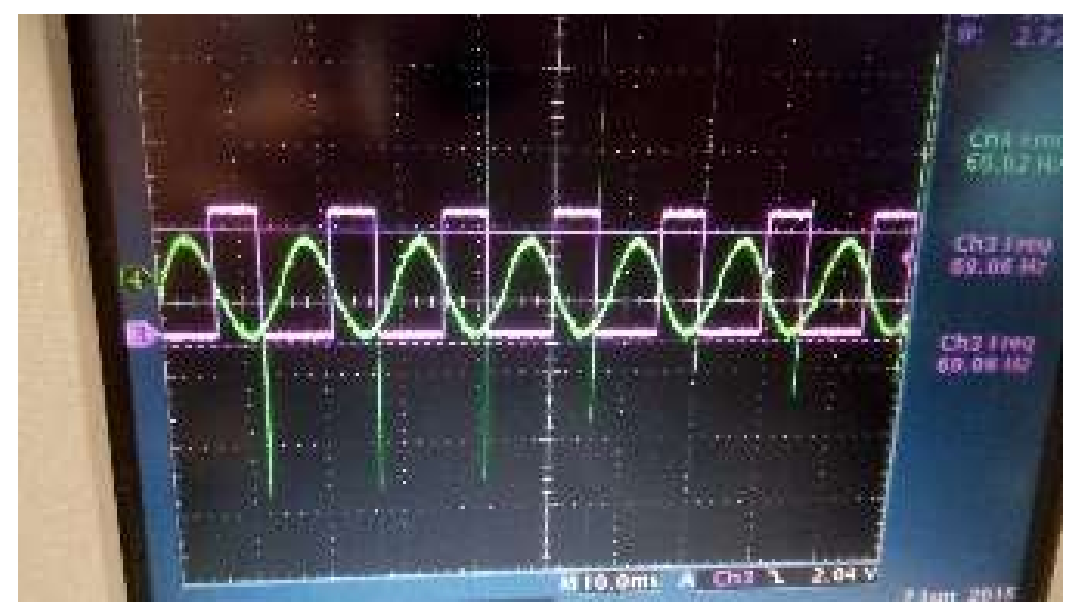

Figure 35. Open-Loop Signals as a Start-up Mechanism 


\subsubsection{Timer Function - Speed Calculation and Signal Generation}

Inside the timer service routine, the DSP read the AB counter and the index counter. The index counter is a counter which is assigned to the $\mathrm{Z}$ signal of the encoder. With each interrupt of the $\mathrm{Z}$ signal, the index counter increments or decrements based on the direction of alternator movement. Using these two counters, the relative position of the alternator could be calculated. Since for speed calculation, a history of the position is required, based on the order of the speed estimator function, the position history in an array of length five is recorded. Then, the alternator speed using Second Order Backward Derivation Approximation is calculated:

$$
F^{\prime}(x)=\frac{3 F(x)-4 F(x-h)+F(x-2 h)}{2 h}+O\left(h^{2}\right)
$$

Equation 1. Second Order Backward Derivation Approximation

Also, the last four values of speed are saved to detect the zero-crossing moment and generate the control pulses. The zero-crossing detection performs as soon as a new speed value is calculated. The detection is strictly based on the following conditions:

- There should be a single change of sign in the last three values of the speed.

- An upper limit is considered for the absolute value of the derivative. Values above this limit are neglected. This helps to remove the noise in the calculation and prevents generation of extra pulses.

Based on the slope of the position before and after the significant change, we can detect a minimum or a maximum position of the linear machine. 


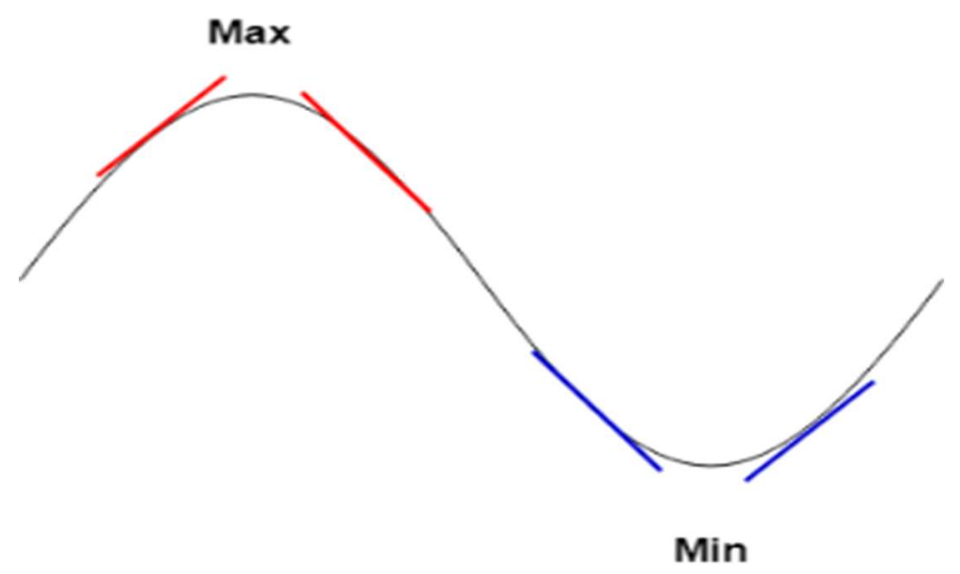

Figure 36. Position Minimum and Maximum Detection

Three signals are expected to be created at speed zero crossings. The PLL pulse, which can be either at minimum or maximum and ignition signals that must be at every peak, one at maximum and the other at a minimum. Therefore, after zero-crossing detection, the required pulses are generated after applying the enforced phase shift of the ignition signals. The phase shift is adjustable by a knob through ADC and makes a delay to start the pulse. This shift is determined based on the engine optimizations. Moreover, the ignition phase shifts can be adjusted independently by two knobs, with two separate shift values, or they can be coupled together and be adjusted by a single value. In the former case, the second signal should be 180 degrees apart from the primary one.

Duty cycles of the three signals and the two phase-shift values of the ignition pulses are made by a time delay defined by a counter which decrements by every timer interrupt.

\subsubsection{ADC Module}

ADC Module has been used for controlling the location of the ignition pulses in relation to the alternator motion to enable sparking at the correct time. Two knobs, or potentiometers, are connected to the ADC pins of the DSP and their values vary by a variable resistor between 0 to $3.3 \mathrm{~V}$, which is the DSP GPIO voltage range. The ADC module starts converting every $1.6 \mathrm{~ms}$, which is adjustable by the ADC control registers. This enables faster control of the ignition pulses for manual control. During the ISR, the 12-bit number, which is generated by the ADC module 
based on the analog input voltage ispassed as a time delay to create the phase shift of the ignition pulses for the engine.

\subsection{5. eQEP Module}

An interrupt happens at every rising edge of the $\mathrm{Z}$ pulse, and the $\mathrm{AB}$ counter should be reset to zero. Every rising edge and falling edge of the A and B signals are counted by the built-in module in the $\mathrm{AB}$ counter of the DSP, which represents the displacements between every $2 \mathrm{~mm}$ poles. By each $\mathrm{Z}$ interrupt, the index counter increments if the motor is moving forward, and decrements if it is moving backward. Summing these two counters results in the real-time position value. Then eQEP ISR checks the direction of alternator movement and increments or decrements the index counter accordingly.

\subsubsection{Interrupts}

The priority of the interrupt plays a crucial role in the success of the code. For example, if the eQEP cannot interrupt when the timer ISR is running, the value of the index counter is not valid anymore. The interrupts are nested manually since they are not nested by default in the correct order. So a higher priority interrupt is able to interrupt a lower priority one. Also, the priorities have been assigned based on our application. The eQEP interrupt has the higher priority since missing any $\mathrm{Z}$ interrupt could cause a $2 \mathrm{~mm}$ inaccuracy in the position. Similarly, the external interrupt is the first priority as well and it never happens with the interrupt of eQEP. They do not happen at the same since one of them occurs at rising edge of the $\mathrm{Z}$, and the other on the falling edge, and the running time of ISR is small in comparison with the width of the pulse. The timer has the second priority and ADC has the third. The justification for the timer and ADC priorities is that $1.6 \mathrm{~ms}$ delay in changing the phase shift does not harm the system performance drastically.

\subsubsection{SPI Communication}

The SPI built-in module sends the position information out to a DAC via SPI communication protocol. The master must send the clock signal with a user-defined frequency, or baud rate, to the slave. The slave is the (Microchip MCP4921) DAC chip. The SPI module shifts the data into the 
send buffer along with an enabling signal that is connected to the chip select of the DAC. The Baud Rate is set to 7.5 MHz, and Data word length to 16-bits.

The SPI is a standard communication protocol between the DSP and external peripherals such as external Input/Output (I/O), peripheral expansion via display drivers, DAC devices, or other microcontrollers. It is a high-speed synchronous serial I/O port that allows a stream of bits with a user-defined length to be shifted into and out of the device at a user-selected bit-transfer rate.

\section{DSP DAC}

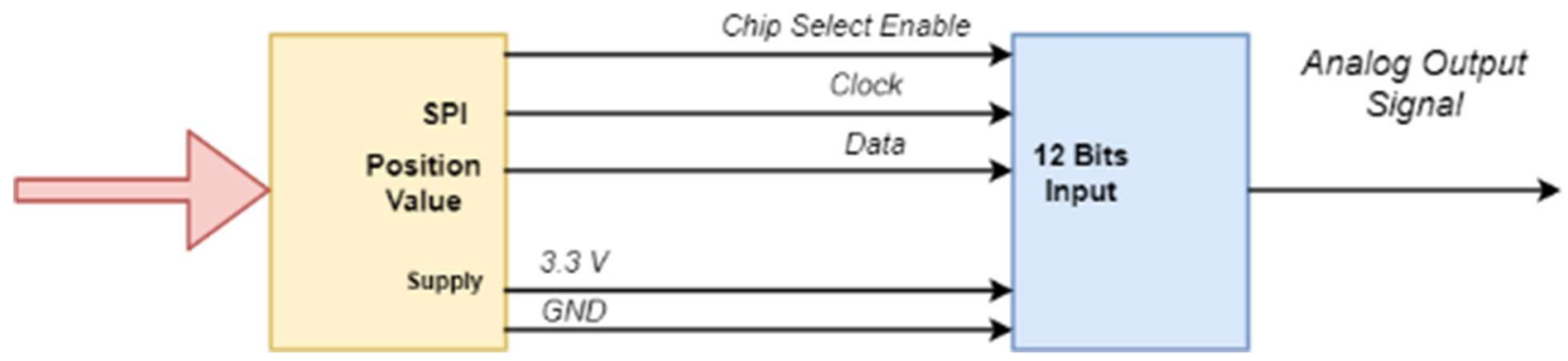

Figure 37. DAC and DSP connections

\subsection{Data Acquisition}

\subsubsection{SCl Communication Protocol}

The availability, reliability, and accuracy of position data are fundamental in this system. It is the basic element in any analysis and control systems. Also, there is a need to display the position during the operation of the OLEA system to make sure that it is functioning as expected and for diagnostics purposes. Therefore, a communication, recording, and displaying scheme is required. In addition to the lack of a reliable data acquisition system, assurance of how smooth the encoder is operating is not known. Therefore, a communication scheme is obligated to observe and use the encoder position values computed by the DSP.

A straightforward, accessible method is required to send out the position or other signals from the DSP to the computer through Serial communication. The communicated data can be used for logging, debugging, or troubleshooting the system. The DSP is equipped with SCI communication 
protocol through the USB-to-JTAG debug probe connected from the DSP to the computer. Because of the limitation of the SCI data transfer speed, a second DSP was utilized to accomplish logging the data. SCI procedure could keep the DSP busy and miss the main tasks or delay the executing of critical functions.

Consequently, the second DSP has been dedicated to only log the data. By adding the junction to the same data which is going from the encoder to the main DSP, and feed it to the data logger DSP, it was assured that the output data is similar to what the main DSP is confronting. The same position calculation method as the main DSP is implemented on the logger DSP as well.

The maximum baud rate that DSP SCI module is able to handle is 230400 bits per second. According to the number of bytes that are supposed to be sent at each sampling time, the rate of sampling is selected as 500 Microseconds. In other words, the DSP computes the position every $500 \mu \mathrm{s}$ and sends the data to the computer with two digits and three decimal numbers. Position calculation and send functions are both called by a timer. To this end, the DSP internal timer has been enabled and set to the desired time period, which is $500 \mu \mathrm{s}$.

This logging scheme is mostly appropriate for offline logging needs and for analysis of the position data.

\subsubsection{Receiving the SCI Data}

A software on the computer side has been developed to receive and record the data sent by the DSP through Serial communications. The software is developed using MATLAB which receives the data and saves it in CSV format. The data can be analyzed or visualized later. 


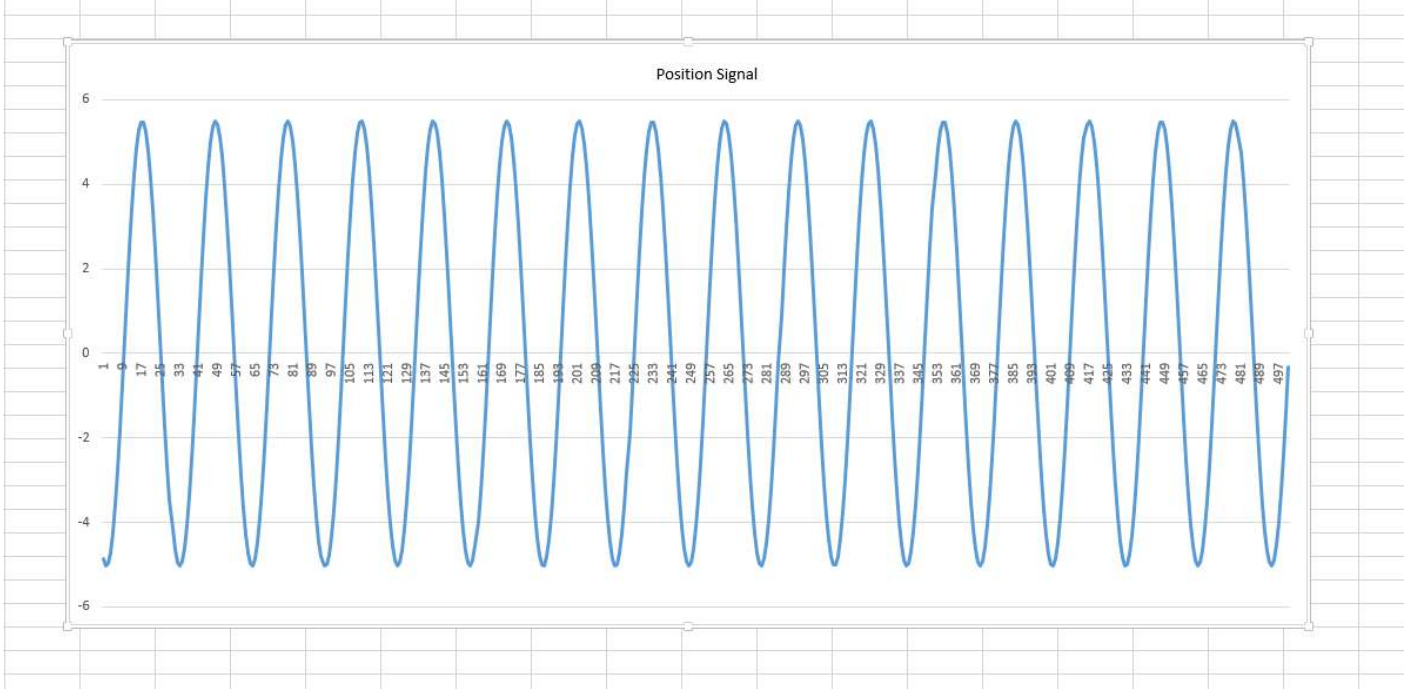

Figure 38. Position Plot from the Data Received through SCI

For easy user interaction with the software, a MATLAB GUI interface has been designed. The GUI let the user start and stop logging data by pressing a button. The received data can be saved on a CSV file automatically by disconnecting the SCI communication port or manually. The data can also appear on screen as soon as it received, but it degrades the performance of the communication on high speeds. The GUI can also send control parameters to DSP while the motor is running. More details will be explained in the following sections. 


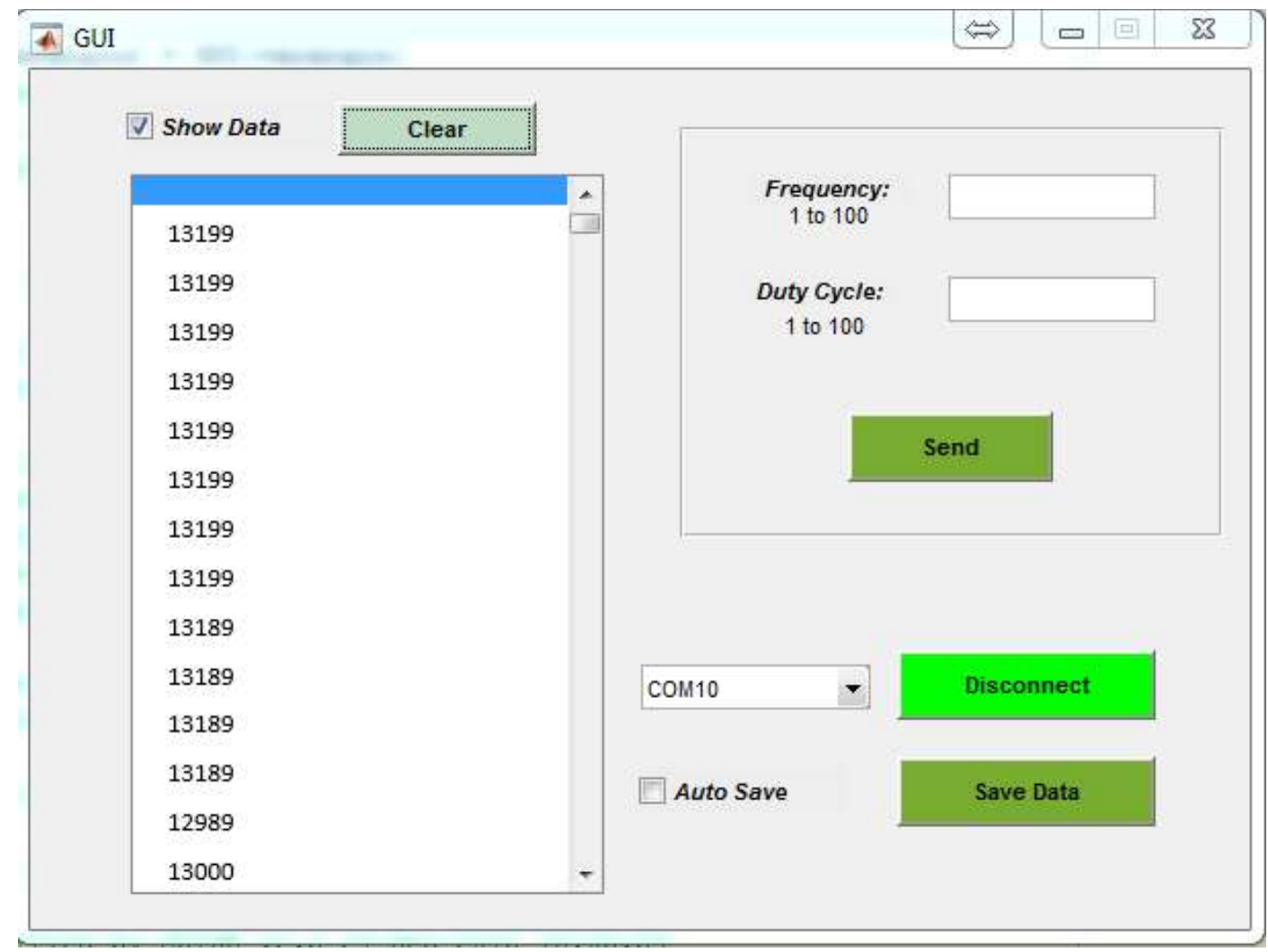

Figure 39. GUI Interface throughout Running

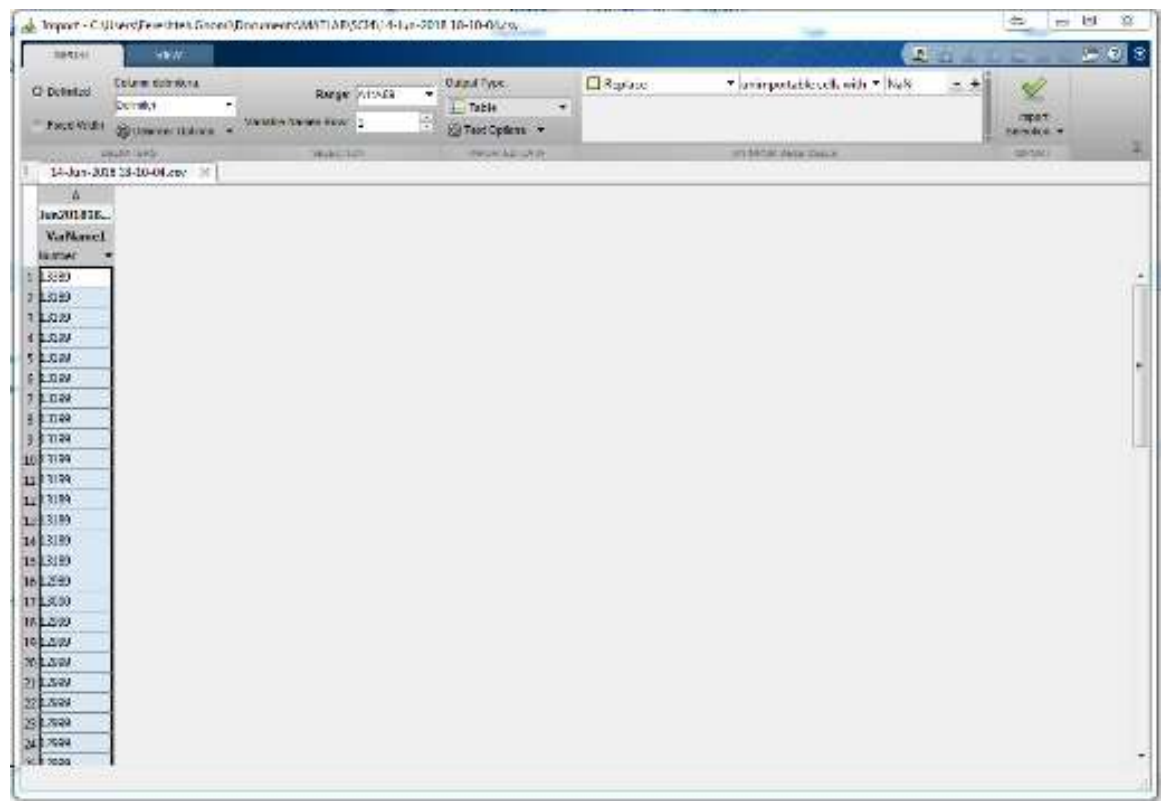

Figure 40. Saved Excel File by MATLAB 
Termite was also used to log the data. Termite is a serial terminal which is faster than MATLAB.

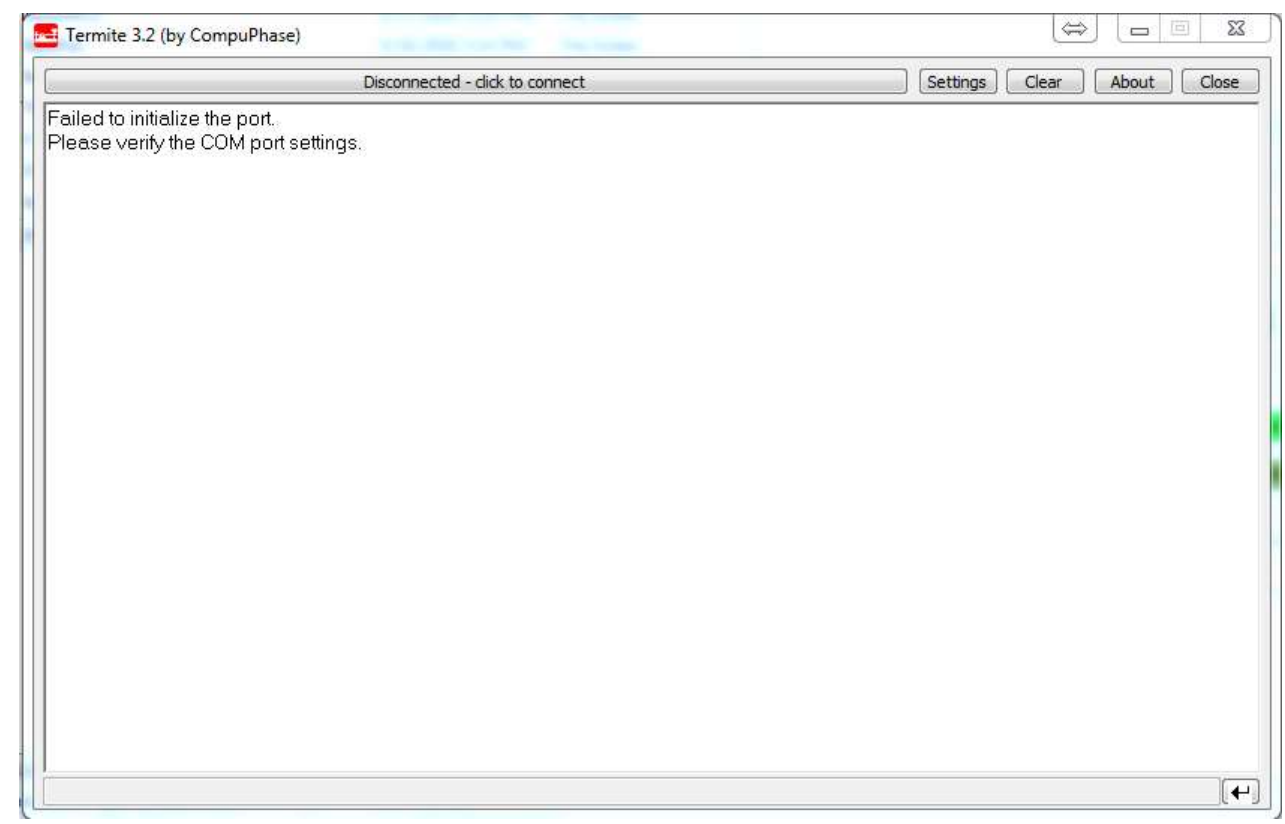

Figure 41. Termite Software Interface

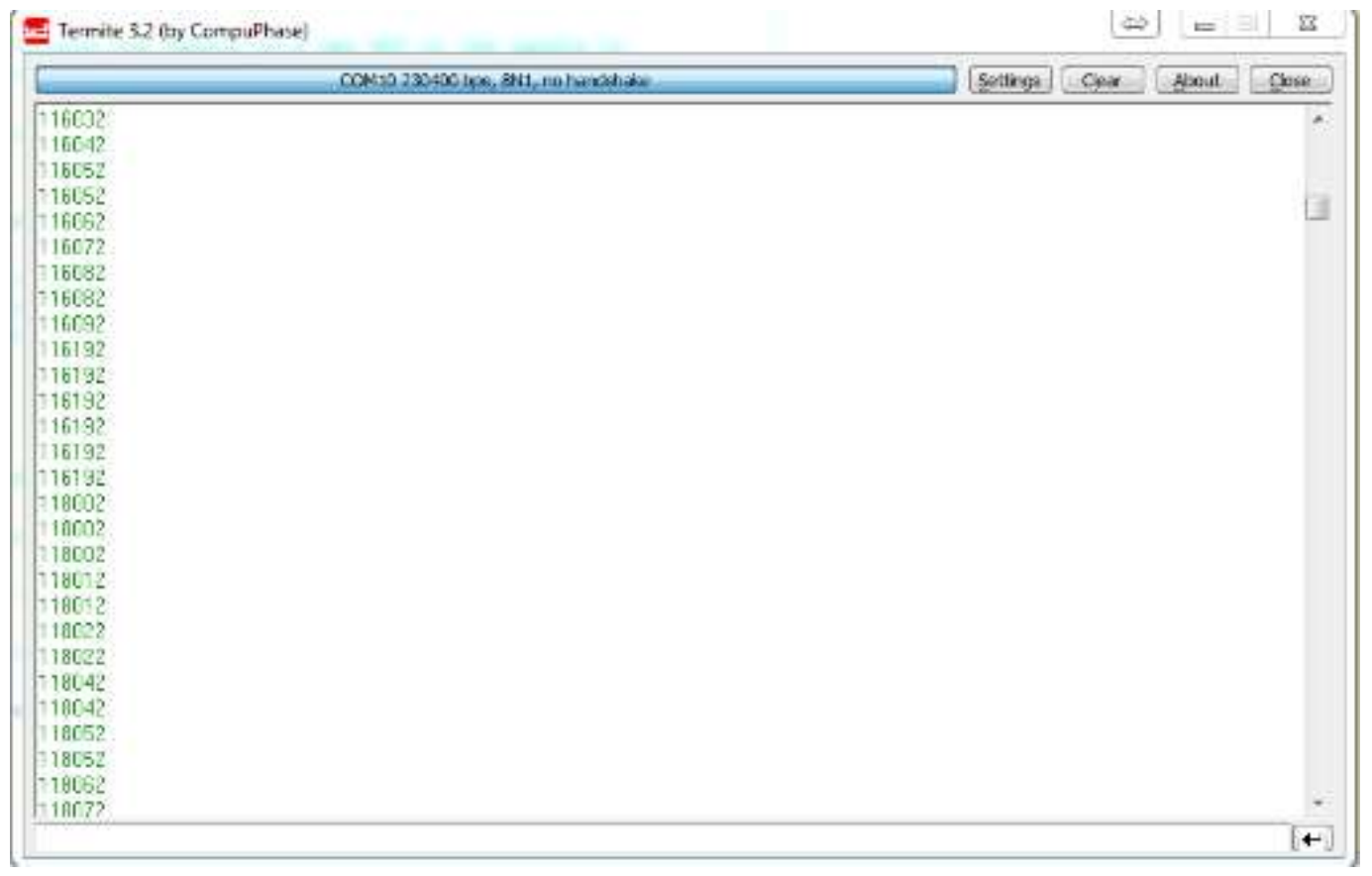

Figure 42. Termite throughout Running 
Logging the data, by the means of our own developed MATLAB-based software or Termite, is a necessity for testing, debugging, and visualization. We logged the data for these purposes constantly. Figure 43. Speed Plot from the Data Received through SCl shows the logged velocity by computer.

By this method, a calculated speed which is a derivation of the position signal is sent to the computer and plotted. The plots proved that the derivation method is guaranteed and creates a smooth Sine wave out of the position Sine wave that can be the fundamental data to make the control decisions and determine the points of starting the needed pulses.

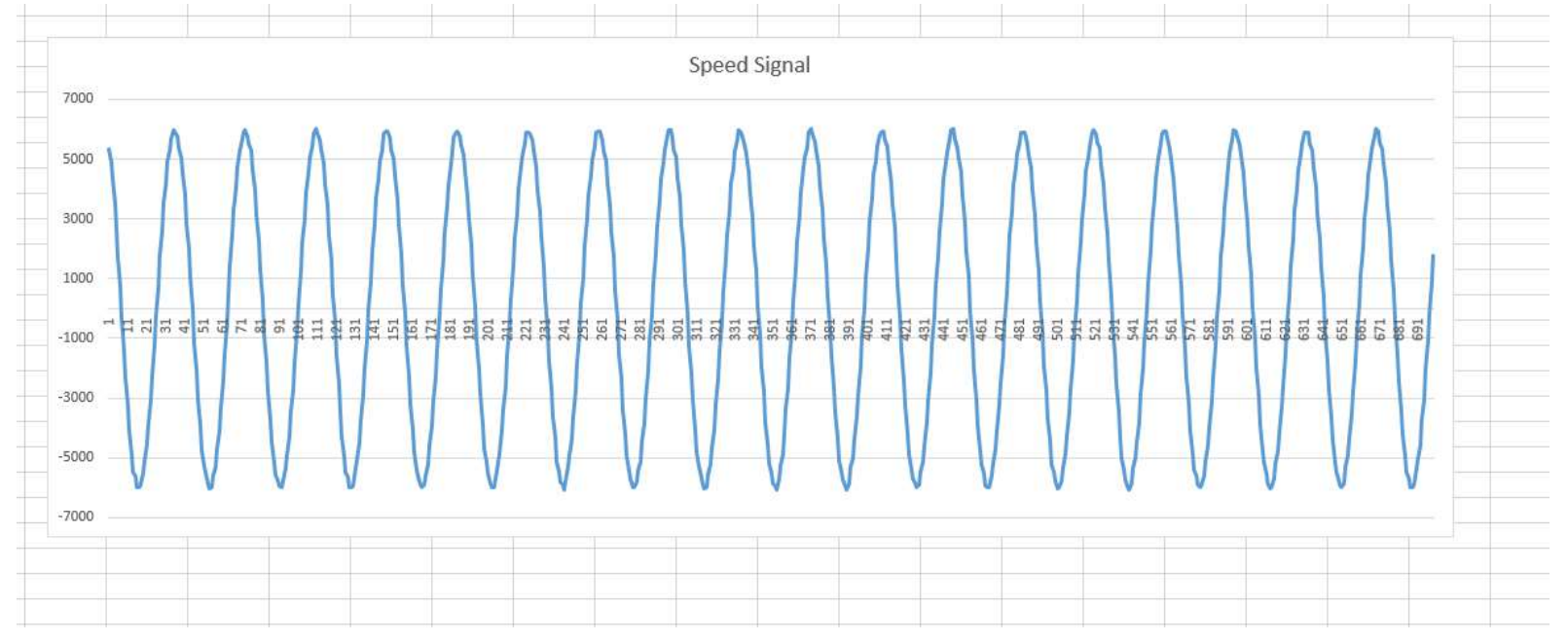

Figure 43. Speed Plot from the Data Received through SCI

Figure 44. Position and PLL Pulses Plot from the Data Received through SCI illustrate the position signal (blue line), and PLL pulses (orange line). The logged data shows the perfect timing of the generated pulses. Note that the logged data communicated by the auxiliary DSP, but it runs the same algorithm to generate the PLL and ignition pulses. Figure 45. Speed and Ignition Signals Plot from the Data Received through SCI also visualize the moment of ignition pulses. These figures also show smooth sinewave position and speed signals as desired. 


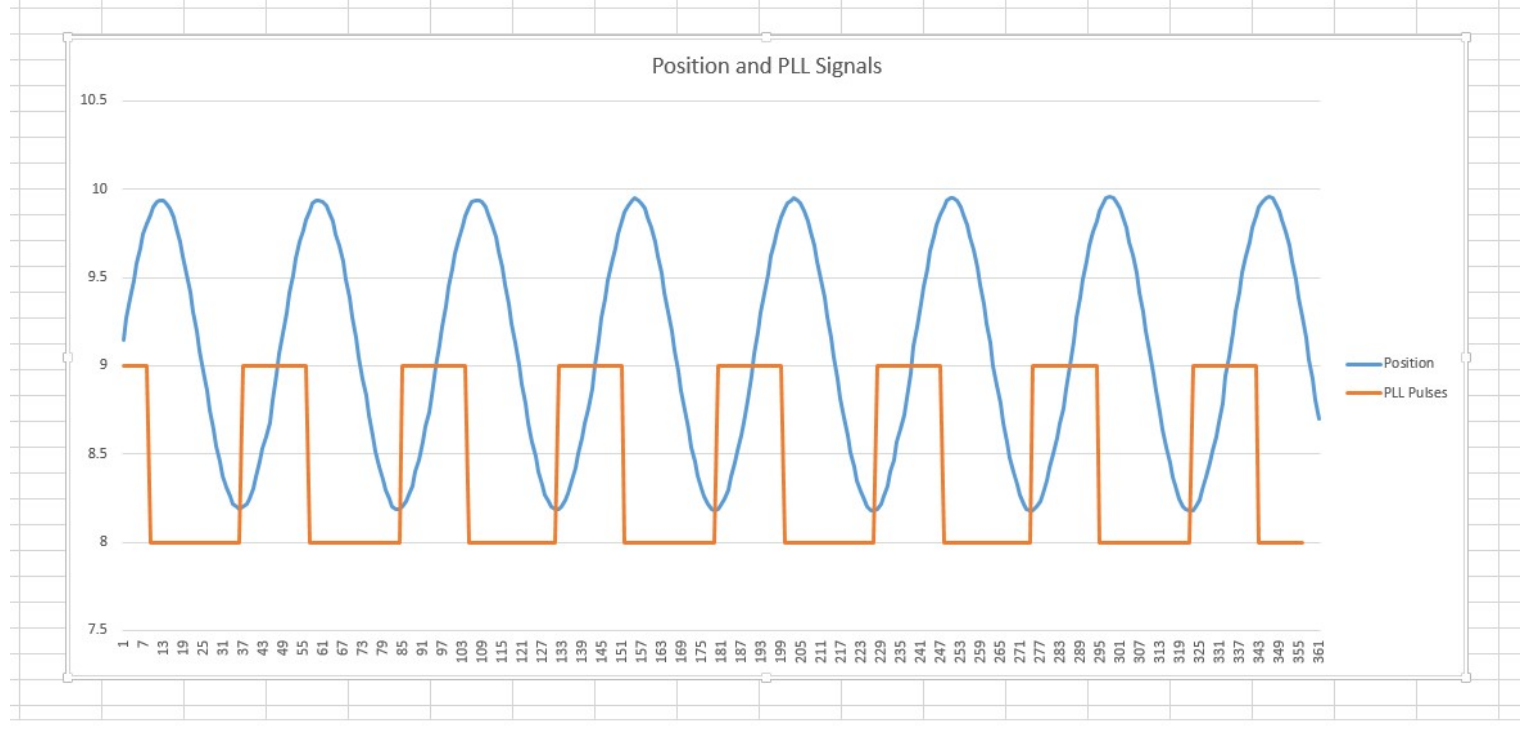

Figure 44. Position and PLL Pulses Plot from the Data Received through SCI

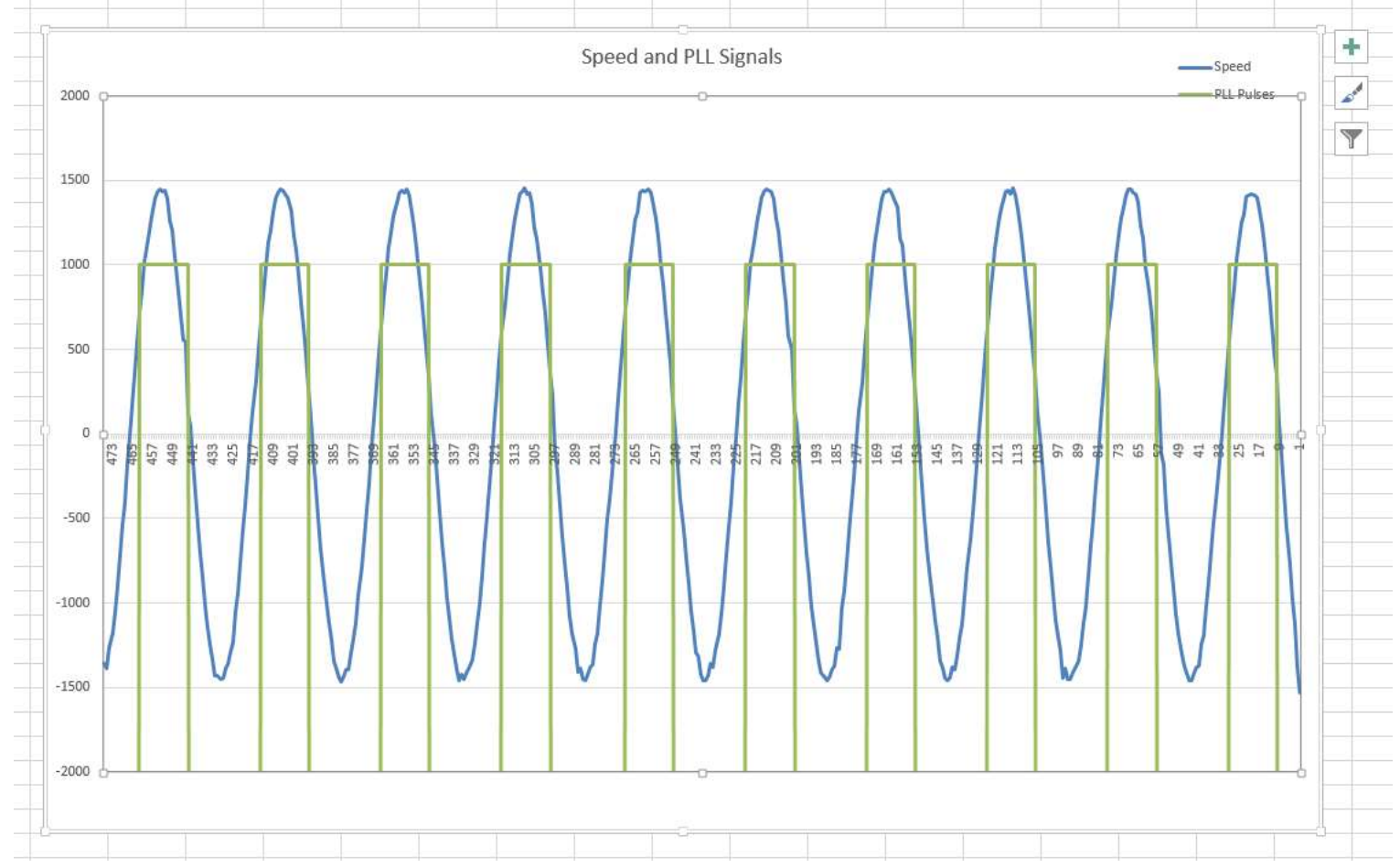

Figure 45. Speed and Ignition Signals Plot from the Data Received through SCI

Displaying the real-time position signal, i.e. online, while the machine is running, and the ability to employ the analog position value coming from the encoder is also essential for system analysis. 
To this end, a DAC chip has been used to convert the position digital data to an analog signal. Employing a high-speed communication protocol, such as SPI, enables us to have a high-resolution data output from the main. For this purpose, we selected Microchip MCP4921 DAC which supports SPI communication.

\subsubsection{MATLAB Interface}

To test or troubleshoot the motor, we can run it in an open-loop mode. In addition, running the motor in open-loop let us find the resonant frequency. To find the resonant frequency, changing the frequency of the driving pulse, while the motor is running is required. In this way, the DSP does not need to be reprogrammed.

A MATLAB GUI has been developed to create a simple interface to communicate with the DSP and tune the controller by changing the desired parameters. The frequency and duty cycle values could be sent by GUI to the DSP. In addition, as we mentioned before, the GUI is able to log the DSP data, display the data in the GUI window, or save it as a CSV file.

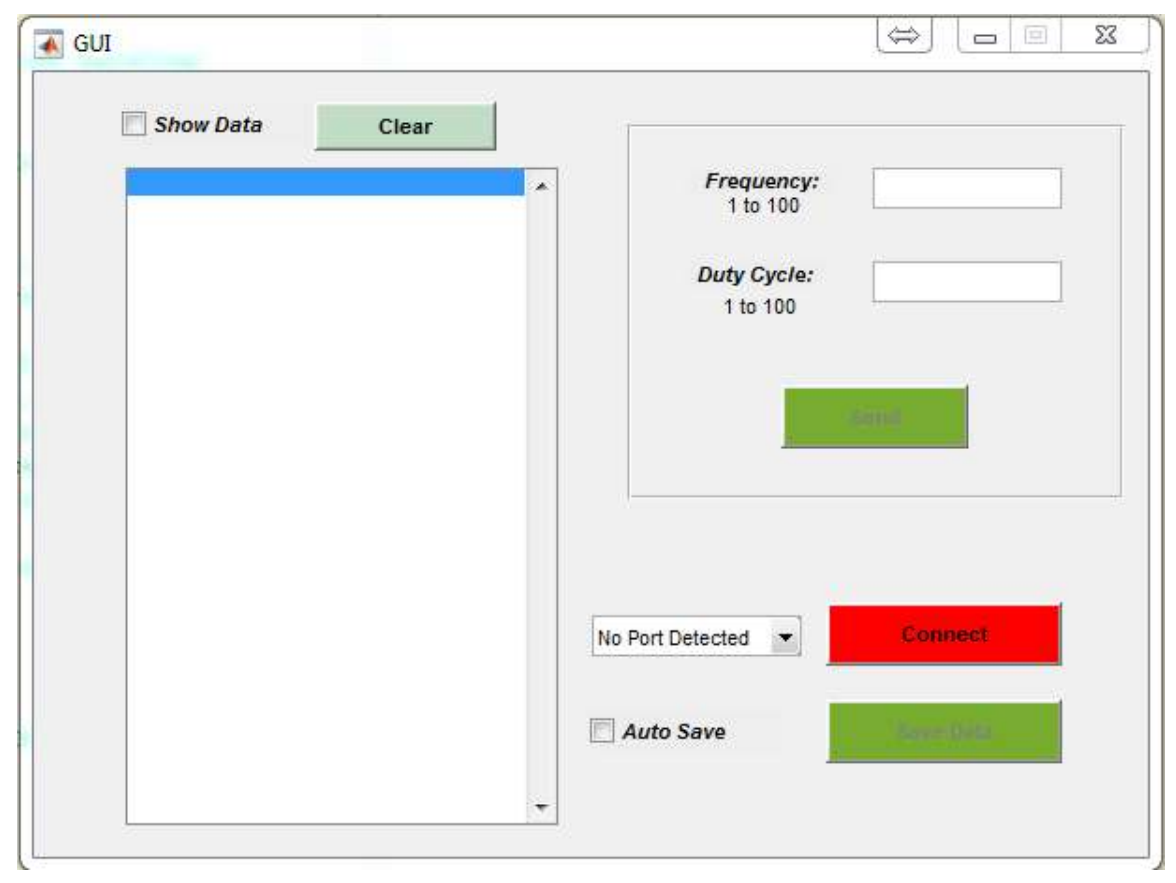

Figure 46. GUI Interface to Command the Open-Loop Run 


\subsubsection{SPI-Enabled DAC Chip}

By the means of SPI communication protocol, and utilizing an SPI-enabled Digital to Analog Converter IC, a fast data acquisition method has been implemented.

SPI high rate of transferring data enables us to send the data with high sampling rate to get a smooth sinewave signal on the oscilloscope. The Baud rate in our implementation is $7.5 \mathrm{Mbits}$ per second. The DSP is the SPI Master while the DAC chip runs as a Slave. A Microchip MCP4921 12-Bit Voltage Output Digital-to-Analog Converter has been utilized with SPI Interface with 20 $\mathrm{MHz}$ Clock Support for this purpose. In addition, the operating voltage of the chip is in range of $2.7 \mathrm{~V}$ to $5.5 \mathrm{~V}$, which let us to power it by $3.3 \mathrm{~V}$ power pin of the DSP board. In this way, we do not need an extra voltage converter to run the DAC chip. The IC has been also mounted on the DSP breadboard for stable and reliable wiring. The DAC needs three signals from the DSP, in addition to the supply voltage, including the Chip Select signal, Serial Clock signal, and the Serial Data signal.

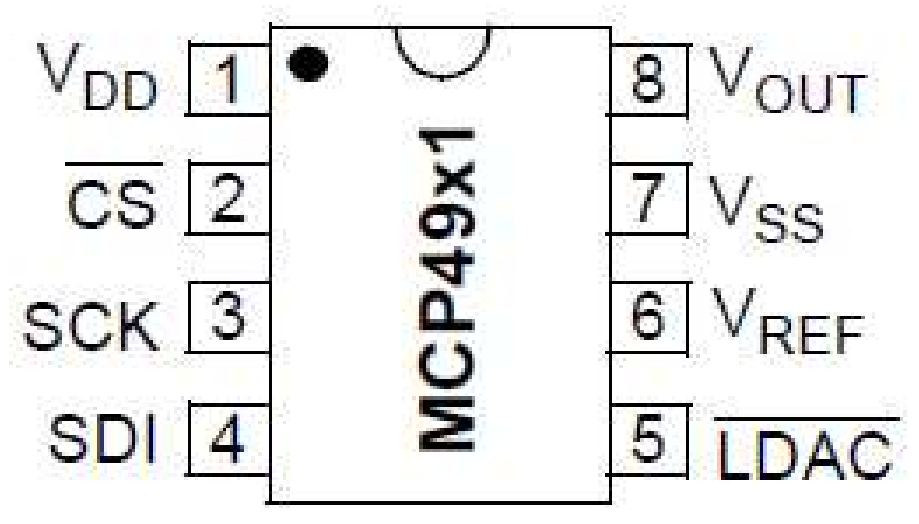

Figure 47. DAC Package Pin Out 


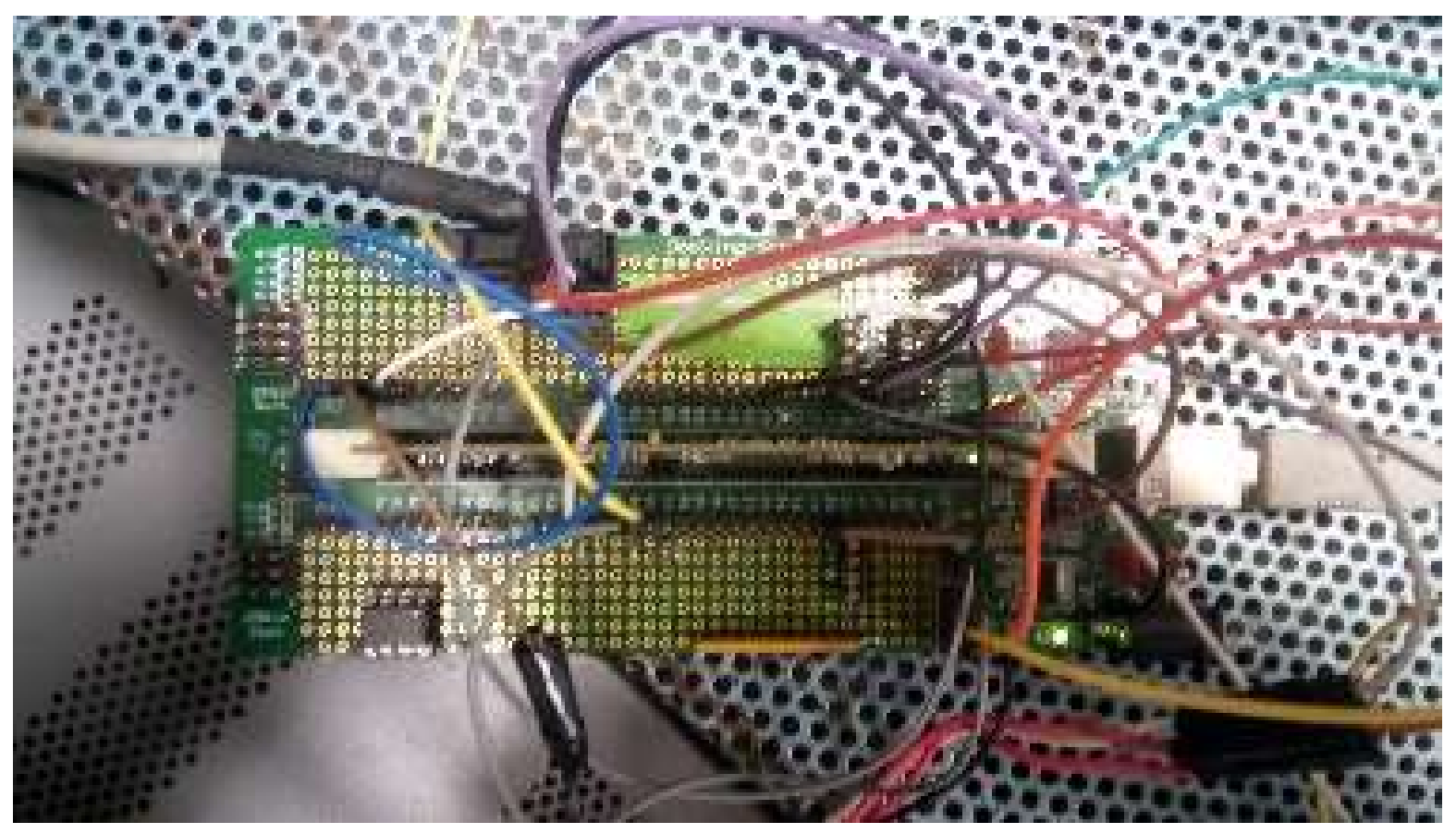

Figure 48. DAC Mounted on the DSP Board

\section{Software Testing}

A complete software testing system is essential to make certain that the software functioning properly before running the machine with the code. The software has been tested module by module. The input signal for every section of the code has been simulated by the DSP and observed by the oscilloscope. The simulated input signal from the DSP or the input value has been sent to the DSP as an input to check that the noted section of the code is functioning as it is expected.

The inputs and outputs of the OLEA control system are sinusoidal and pulse waveform signals. The output is acquired by generating the similar input signal.

The challenge in testing and troubleshooting of the OLEA system is the accuracy and simulation of the encoder output signals. Since the output signals need to be generated according to the motion. To simulate the encoder position signals and check the encoder performance and the entire control process, a test bench has been provided. The test bench is a reciprocating saw that runs with a fixed displacement. The linear motion of the reciprocating saw gives the required position signals to test the code and encoder performance. 
The encoder outputs (position signal) are connected to the DSP and the DSP communicates the output value, which is the position or speed value to the computer through SCI or SPI communication protocols. Also, the control output waveforms and the DSP inputs were displayed on an oscilloscope.

A position sensor has been installed still on the body of the saw and the magnetic strip is mounted on the moving part of the saw. The test bench is shown in Figure 49. Therefore, by the means of the reciprocating saw and controller input signal simulations, the controller outputs has been observed or logged on the computer. The output signals and the data have been analyzed to evaluate the system performance and accuracy.

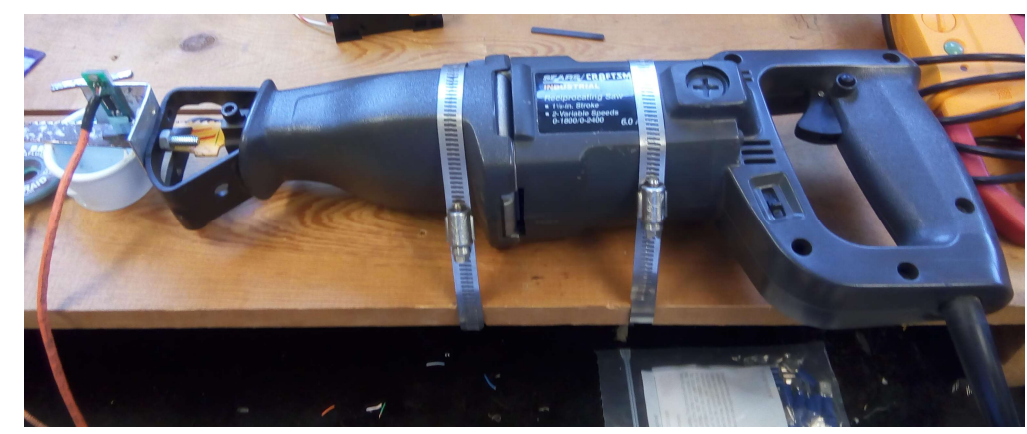

Figure 49. Reciprocating Saw as a Test Bench

\section{Troubleshooting}

\subsection{Part 1}

A comprehensive testing and troubleshooting is required to make sure that a system is performing well in all conditions. We performed that by logging different parameters and signals in a computer and in an oscilloscope. This process is critical for debugging an observed or undesirable behavior from the control system, such as extra or missing PLL or ignition pulses from the generation of the noisy position signal. One of the most critical faulty situations in our system happened when the translator reached the end of on one of the encoder poles, stopped moving, changed its direction, and starts moving on the opposite direction. When changing direction happens at the pole, for example in case of moving forward, the index counter increments at the rising edge of 
the $\mathrm{Z}$ pulse inside the ISR. But before it reaches to the falling edge of the $\mathrm{Z}$, the direction changes and the alternator starts moving in the reversed direction. In this situation, no extra $\mathrm{Z}$ pulse is generated to decrement the value of the index. As a result, the extra increments, that were supposed to be compensated by decrement after direction reversion, can be accumulated during the time and makes a gradual shift in the position sine wave. The DSP eQEP module is not able to detect this faulty behavior since the $\mathrm{Z}$ interrupt can only happen at the rising edges of the $\mathrm{Z}$ pulse. To overcome this issue, an external interrupt was employed. The new external interrupt was triggered by the $\mathrm{Z}$ pulse as well, however in a falling edge.

Therefore, the $\mathrm{Z}$ signal is fed to the corresponding GPIO pin of the external interrupt which is set to trigger at the falling edge of its input pulse. To be able to correct the index signal in case of direction change on encoder poles, we record the direction of the movement in rising edge of the $Z$ signal, inside eQEP ISR. However, inside the external interrupt ISR, which happens on the falling edge of the $\mathrm{Z}$ signal, we compare the instantaneous direction of alternator movement with its recorded value on the rising edge. If the direction is changed, then we correct the index counter be increment or decrement based on the direction of movement. Otherwise, the index signal left unaltered.

\subsection{Part 2}

The explained control scheme was working for the first couple of month as expected. However, after running the machine for a few months, some problems appeared in the generation of control signals, such as missing the PLL and ignition pulses and generating some extra false pulses in wrong positions. The missed or false ignition pulses degraded the engine performance. 


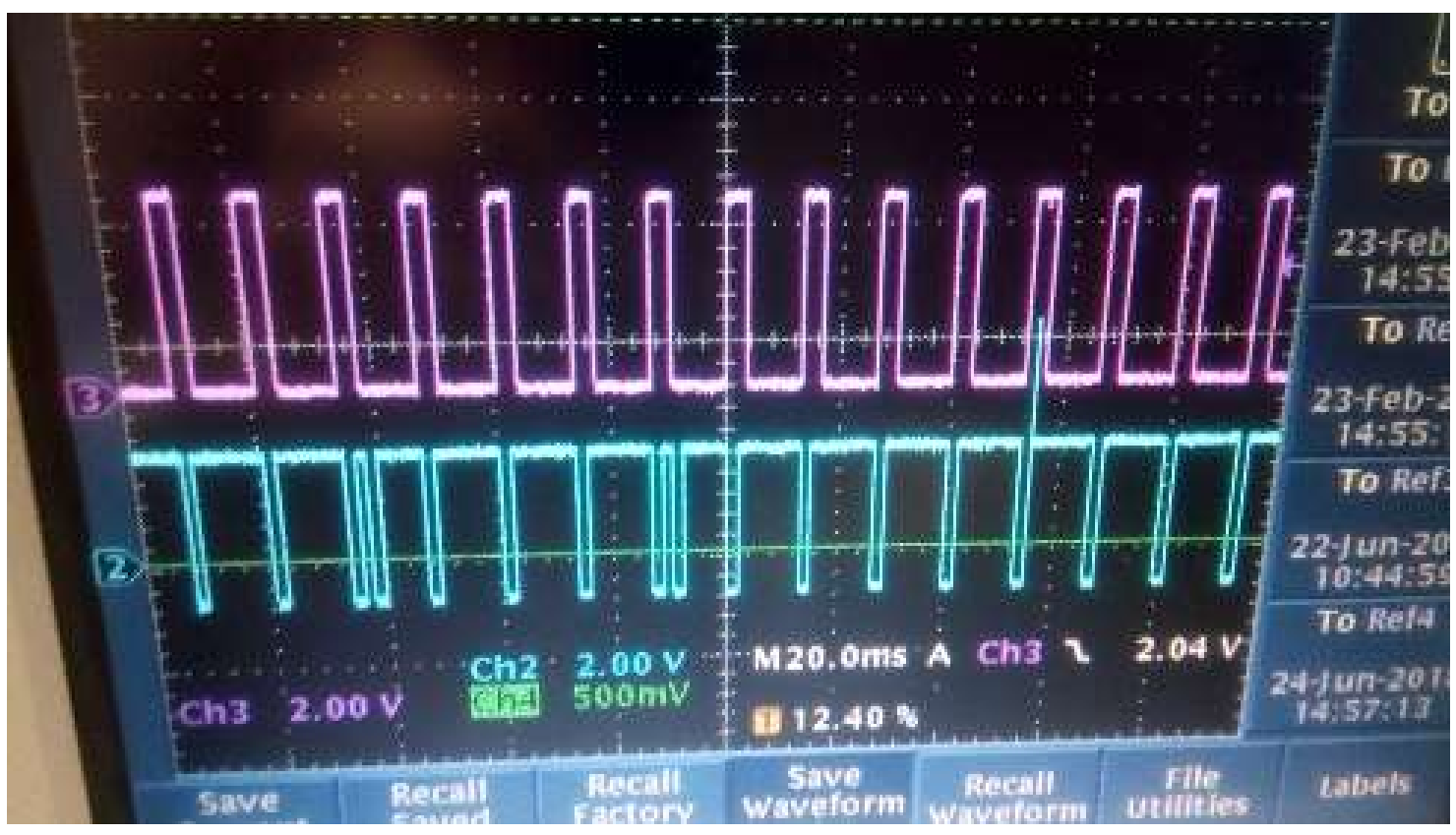

Figure 50. Extra PLL Pulse on the Oscilloscope

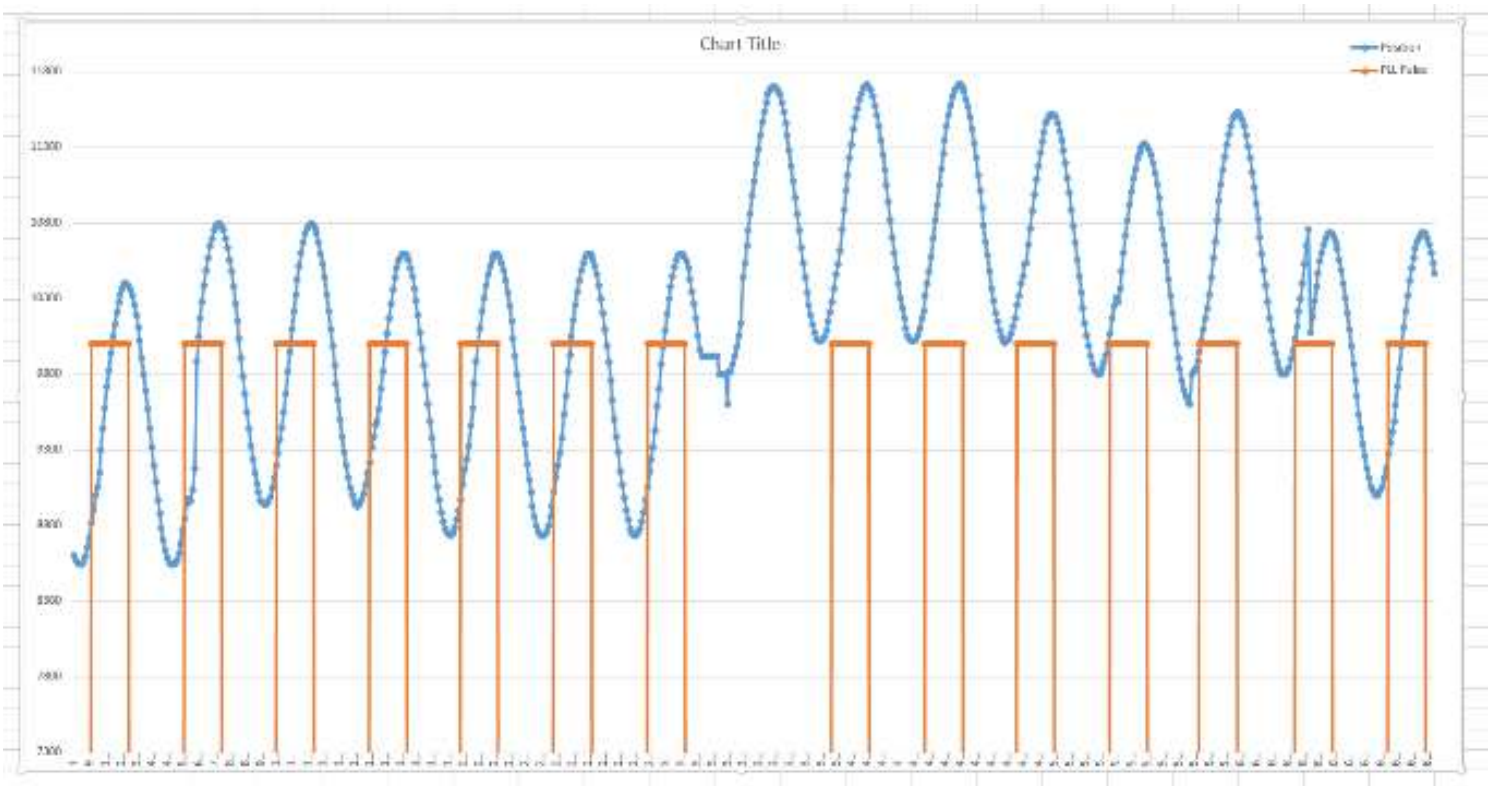

Figure 51. Missing a PLL Pulse 


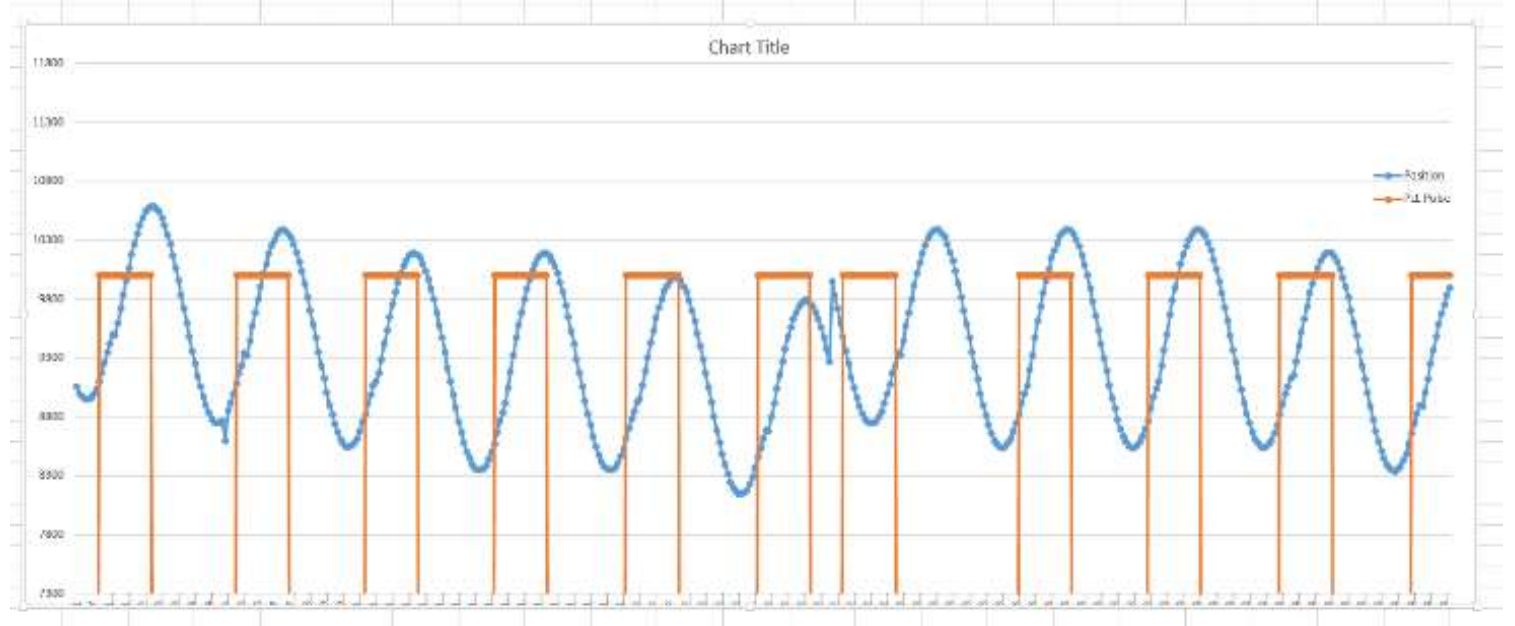

Figure 52. Extra PLL Pulse

We deeply investigated the issue by logging the signals and parameters of the motor, such as the position signal along with the three encoder outputs, both in offline and online manner. Eventually, the position waveform measured by the DSP was found to be not smooth, with too much noise and jumps. Despite the ability of the code to handle the noise to some extent, the level of the noise was so high which resulted in misdetections in considerable situations.

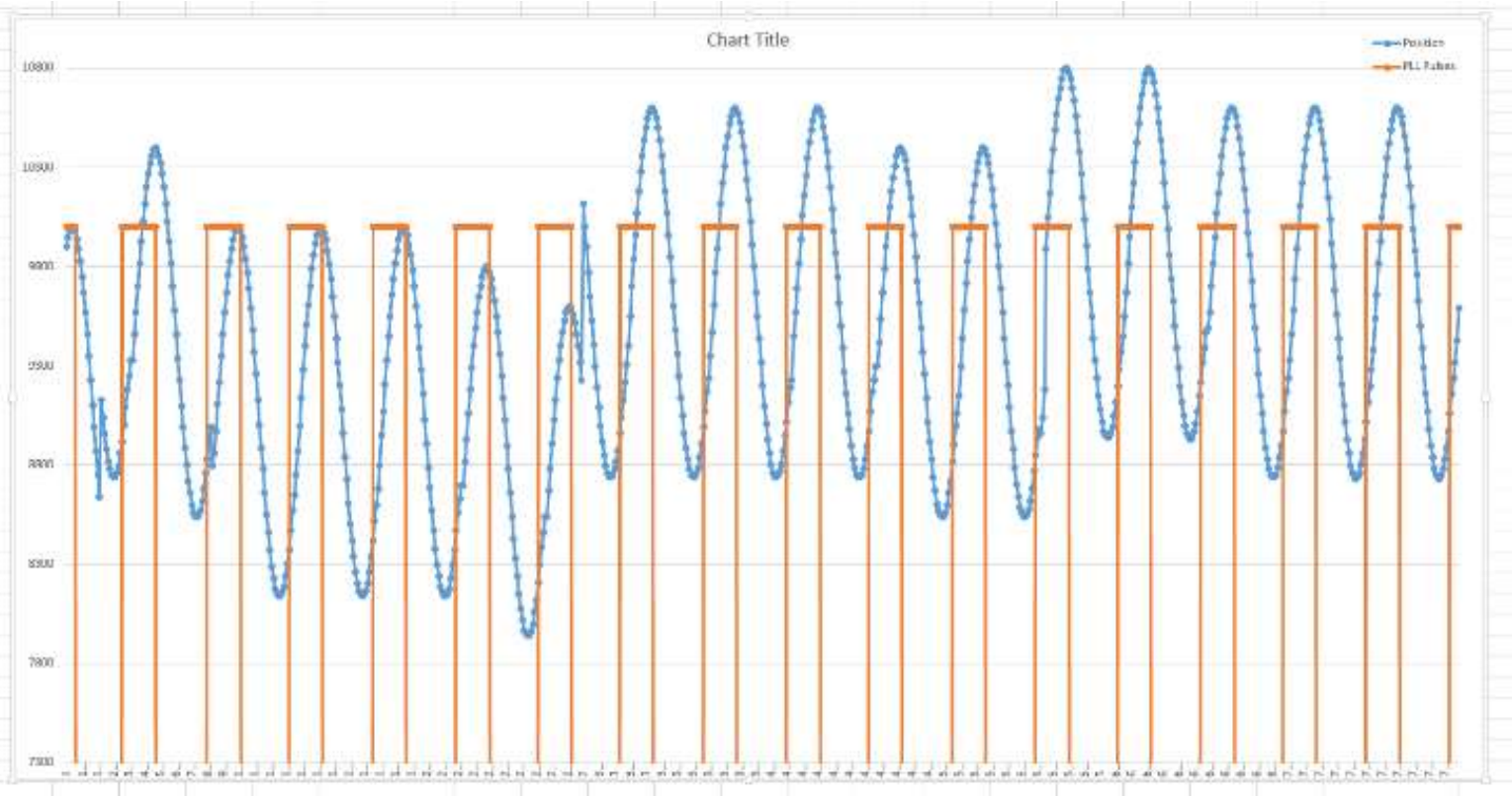

Figure 53. Unsmooth Position Signal but Handled 
Figure 53. Unsmooth Position Signal but Handled illustrate the unsmooth position sine wave, which is shifted and jumped upward and downward unexpectedly. Since each Z interrupt represents a $2 \mathrm{~mm}$ movement of the alternator, any extra interrupt triggered by the noise could result in a jump in the position signal. After further investigation, running diagnosis and monitoring the $\mathrm{Z}$ pulse coming from the encoder, the source of the noise identified to be the switching of the inverter that causes extra interrupts to be triggered on the $Z$ pin of the DSP. The external interrupts of the DSP are too sensitive to noise, even in the presence of pull-down resistors, and switching noise was making a lot of false interrupts on the DSP pin. Consequently, the DSP was incrementing or decrementing the index counter incorrectly.

In other words, the switching noise disturbed the system by triggering false interrupts, which resulted in an index miscounting. This miscalculation is the main source of the shifts, spikes, and jumps in the measured sinewave signal of the position. Note that this spikes on the position signal are exaggerated on the speed calculation, which caused missing or extra signals. The shifts also accumulate during the time. This accumulation of error caused the index counter to overflow or underflow which is interpreted as a huge jump in the signal.

To reduce the level of noise, the inverter has been replaced. The new inverter has higher output capacitors to reduce the current spikes at switching times. Also, the turn on/off time of the new IGBTs are larger and it can impact the switching current spikes. Even though inverter replacement reduced the level of the noise, but the problem was not solved for higher currents. Hence a new control scheme to overcome this problem was developed eliminating the interrupts which was susceptible to these switching noises. This scheme is described in detail in the next section.

\section{Results}

\subsection{Elimination Z Signal}

The linear encoder gives A, B and Z signals to the DSP. Z signal provides the $2 \mathrm{~mm}$ displacement interrupts. A and B signal counter in DSP increments in its forward movement and decrements it in its reverse direction. The $\mathrm{Z}$ indicator is a correction and resetting mechanism for $\mathrm{A}$ and $\mathrm{B}$. The $\mathrm{Z}$ fixes the measured position every two millimeters and resets the counter. 
Due to the high sensitivity of the external interrupt from $\mathrm{Z}$ pulse to the noise, the dependency of the control procedure to the $\mathrm{Z}$ pulse makes it unreliable. Therefore, the $\mathrm{Z}$ signal has been eliminated from all the calculations. Therefore, the entire control algorithm developed was based on only the $\mathrm{A}$ and $\mathrm{B}$ signals from the linear encoder. Even though the $\mathrm{Z}$ signal can correct the miscalculation of A and B signals, its elimination does not degrade the performance of the control system, as any mistake in A and B signals results in a small error on the measured position, i.e. 5 micrometers. This level of miscalculation is completely handled by the code and does not cause extra or missing pulses. On the other hand, since the movement of a linear motor is reciprocating, this small error does not cumulate through the time.

By removing the $\mathrm{Z}$ from the position calculation, the measurement is only based on the $\mathrm{A}$ and $\mathrm{B}$ signals, and the only counter in the DSP counts the number of observed edges of these two square waves. This counter gives the final value of the position at every instance. The.

This method is working properly and it is reliable since the system does not rely on missing or extra $\mathrm{Z}$ pulse. This scheme has been tested extensively by running the OLEA as a motor as well as in generator mode.

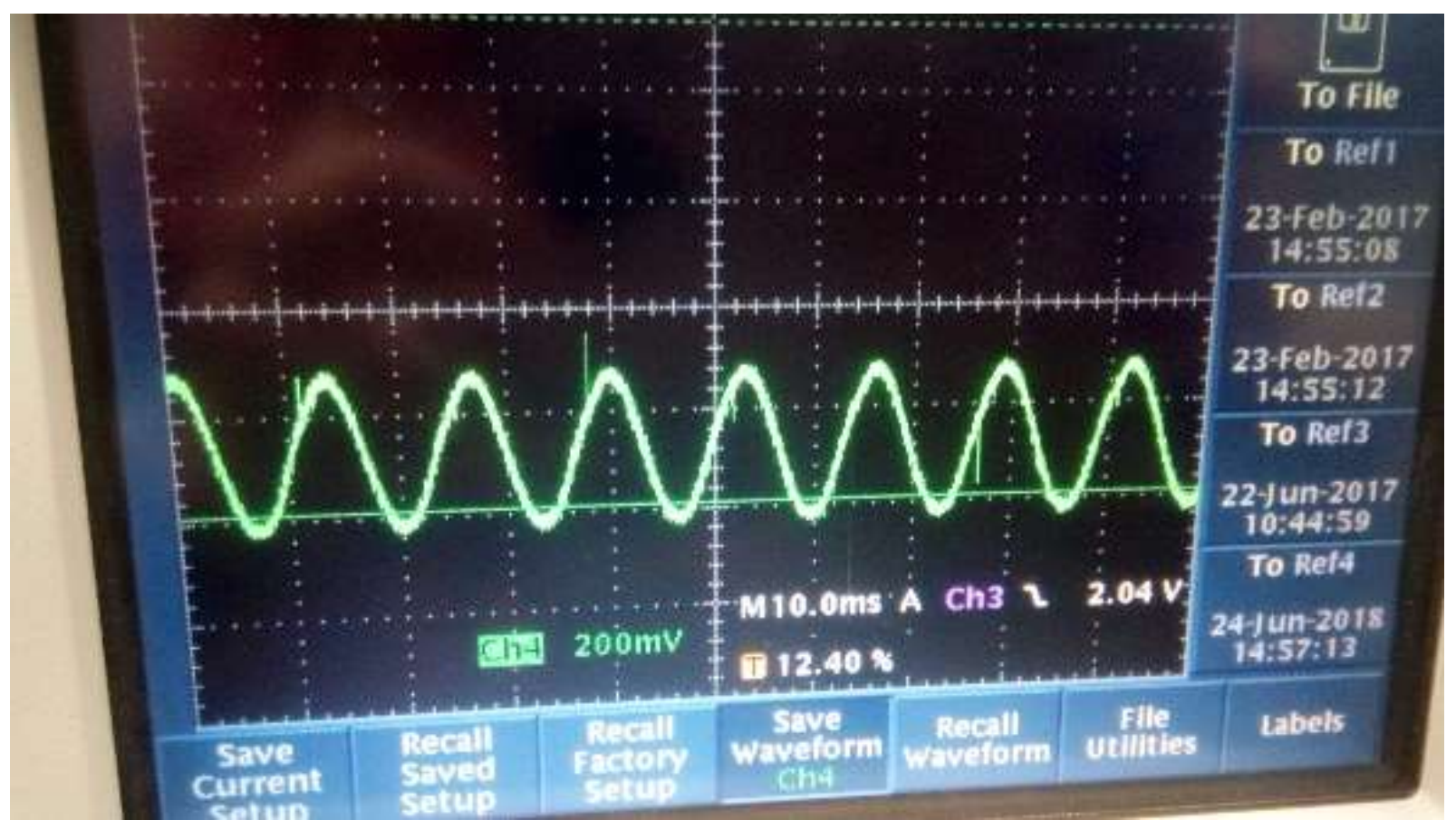

Figure 54. The Position Signal from the Z Elimination Code on the Oscilloscope 


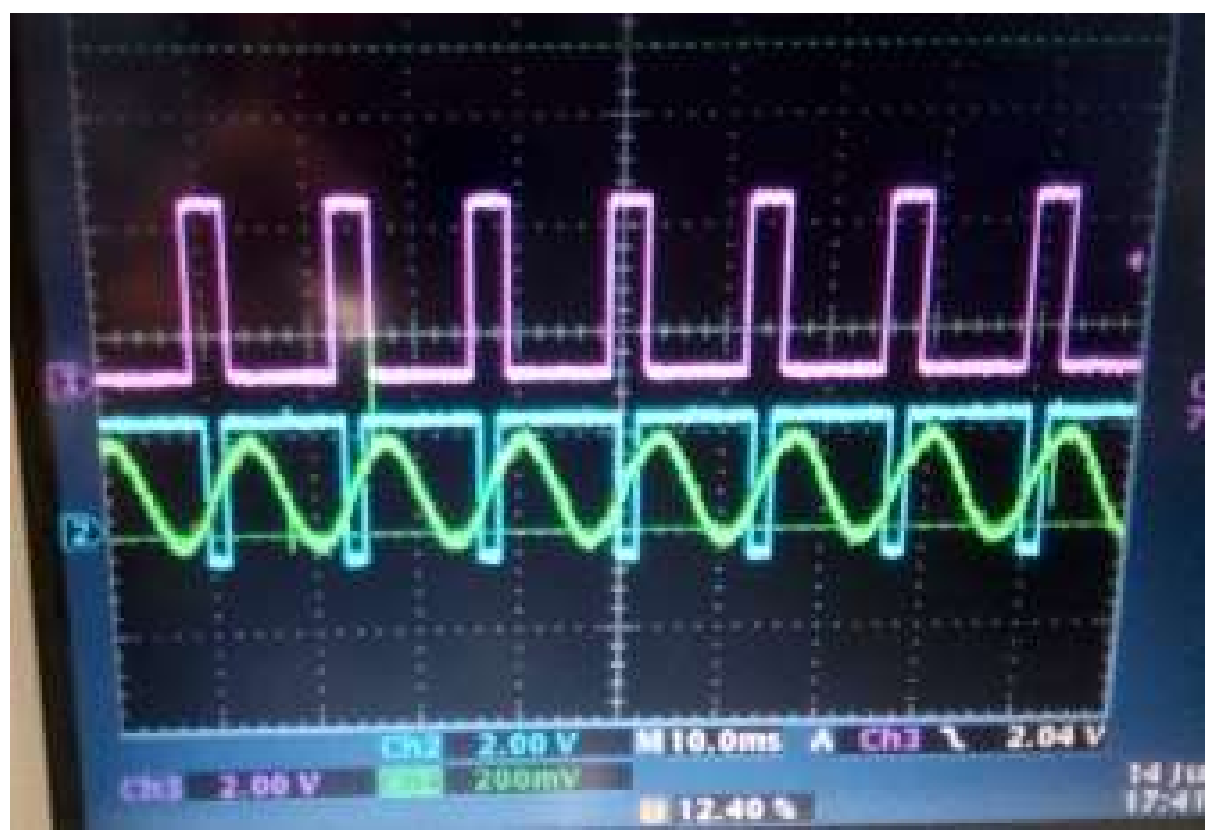

Figure 55. Position, PLL, and Ignition Signals from the Z Elimination Code on the Oscilloscope

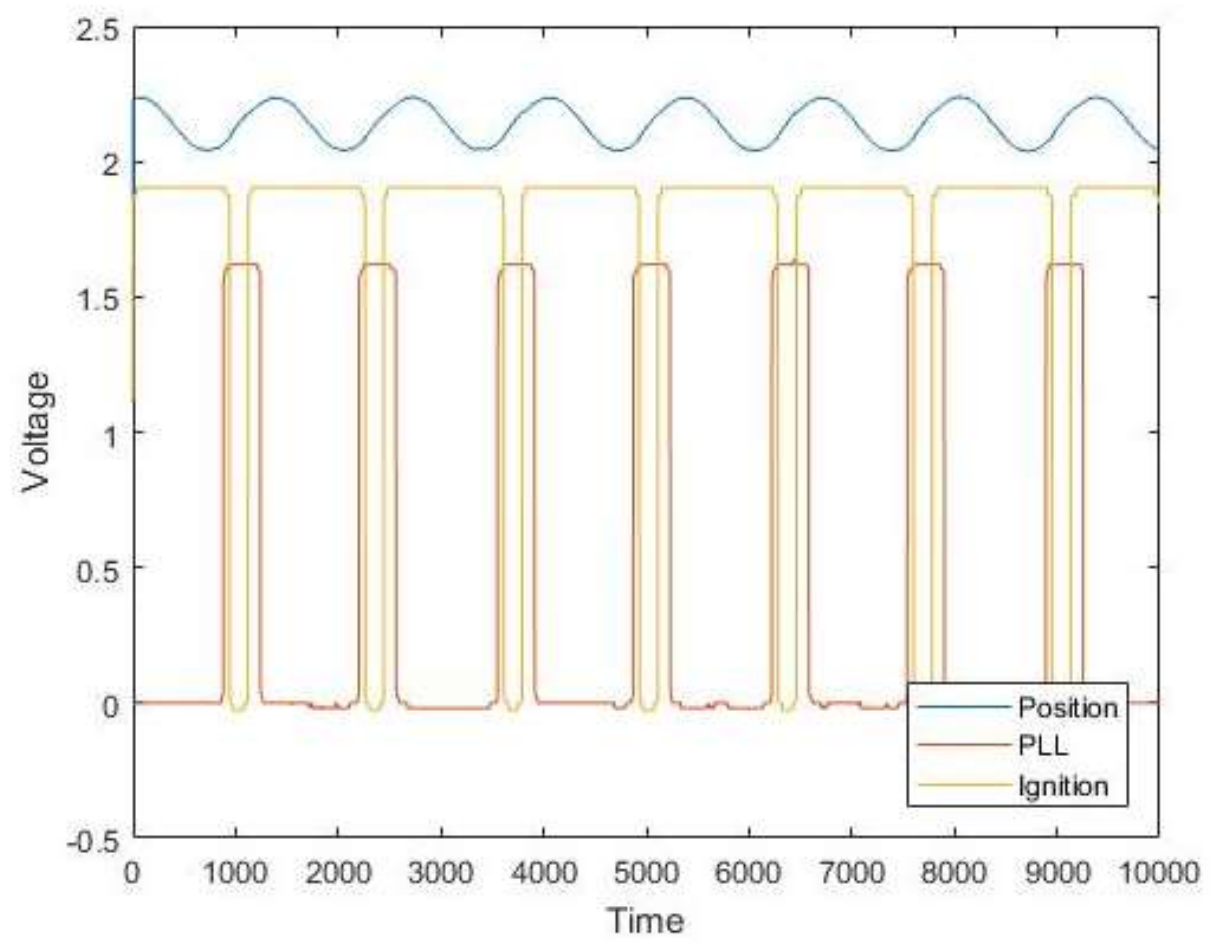

Figure 56. Position Signal, PLL Signal, and Ignition Signal Plot from the Z Elimination Code 
The only drawback of removing the $\mathrm{Z}$ from the position calculation is that finding the absolute position of the alternator is not possible. However, our control strategy is developed based on the translator speed data, and therefore, the absolute position value is not required in our application. In addition, in the linear machine due to the reciprocating motion, this method could provide an accurate enough displacement measurement. 


\section{Conclusion}

A software-based PLL control system has been implemented as a start-up for the OLEA machine. The PLL control system can operate OLEA machine both in motoring mode and generating mode. Once the required compression for the engine is achieved, engine starts running and the control system switches from the motoring mode to the generating mode. Ignition signals required to control the engine during the generator mode have been generated by the PLL control system. The PLL signals have been generated based on the position signal. The position data comes from the incremental linear magnetic encoder, mounted on the linear machine shaft.

A DSP has been utilized to perform all processing, analysis, and control tasks. The DSP receives the input data from the linear encoder and generates the control signals while sending out the position value through a DAC channel. A DAC IC chip has been employed to convert the digital output to analog and make it available outside of the DSP. Moreover, the position and speed signals are available to be analyzed or displayed by establishing two communication protocols to send out the data from the DSP.

An inverter board has been designed to commute the current through the phase windings and provide the essential voltage and current to drive the machine.

Some of the issues involved in developing the inverter - DSP system has been solved through software schemes. The switching noise from the inverter affected the linear encoder sensor performance. To overcome this challenge, the $\mathrm{Z}$ signal from the encoder has been eliminated from the entire algorithm. By applying this change, a reliable and stable control signal without any missing ignition pulses has been achieved.

Overall a complete control system required to run the OLEA Linear machine has been developed. 


\subsection{Future Works}

Some of the future works involved in developing the OLEA system from this work have been discussed below:

- The engine piston in the OLEA is free to move beyond its top dead center (TDC) and bottom dead center (BDC) since the piston motion is not bounded by a crankshaft. The TDC position must be controlled to ensure sufficient compression for the ignition and efficient combustion while avoiding excessive pressure inside the cylinder. Similarly, the BDC position must be controlled to ensure efficient exhausting of the cylinder. The stroke should within a reasonable range to prevent it from contact between the piston and the cylinder head. Consequently, the controller is expected to ensure a stable and smooth engine running. This situation could be detected by monitoring the displacement of the linear alternator shaft. The engine operation, energy conversion and efficiency evaluations are through compression ratio and the velocity of the piston (or displacement of the alternator). [29]. Hence, by controlling the compression ratio, energy balance between continuous cycles can be controlled and assure a stable and smooth engine running.

- The startup with PLL is to reach a specific displacement. Once that displacement is reached, motoring operation should be terminated and the PLL operation should switch to generation scheme. Therefore a mechanism is required identify the switch from motoring mode to generating mode to stop the PLL signals, which involves cutting off the power supply from the machine and let the engine takes over running the machine. Disconnecting the power from the machine is done manually so far, however performing this by the controller automatically is more reliable and efficient.

- The controller could be equipped with an LCD to display the average maximum displacement in a second. The LCD provides an accurate value of the displacement in real time without measuring the position signal on the oscilloscope.

- To $\log$ the position information on the computer and use it later to analyze, or troubleshooting, currently a laptop is needed to communicate with the DSP to receive and save the data. However, by recording the data on an SD card directly from the DSP, there is no need to keep the laptop connected to the DSP while the machine is running. 
- The fuel flow should be controlled by the controller according to the displacement. When the displacement changes, the fuel flow to the engine could be adjusted automatically based on a table of data or a formula.

- When the springs or parts inside the machine breaks, the frequency of operation of the OLEA varies from its original resonance condition. For the sake of safety, the DSP could check the frequency continuously and stop the machine immediately if a major change in the frequency happens. This protection can be carried out by the means of a vibration sensor as well.

- Due to the increase in cost, size, weight, maintenance, and complexity of the system and decrease in reliability, sensorless algorithms are offered to overcome the drawbacks of employing the sensors. There are different sensorless techniques to extract the position data while avoiding to utilize a sensor. Basically, the main idea behind such sensorless approaches is to probe or monitor the phase's terminal voltage and current and process it. Therefore, by measuring the electric quantities, obtaining the information concerning the motion can be done. In this way, the primary measurement is the windings signals which map the relationship between the position and the present electrical waveforms and are easily accessible. 


\section{Reference}

[1] Boldea, I., and S. A. Nasar. "Linear electric actuators and generators [C]//Electric Machines and Drives Conference Record 1997." In IEEE International, pp. MA1-1. 1997.

[2] Famouri, Parviz, William R. Cawthorne, Nigel Clark, Subhash Nandkumar, Christopher Atkinson, Richard Atkinson, Thomas McDaniel, and Sorin Petreanu. "Design and testing of a novel linear alternator and engine system for remote electrical power generation." In Power Engineering Society 1999 Winter Meeting, IEEE, vol. 1, pp. 108-112. IEEE, 1999.

[3] Cawthorne, William Robert. "Optimization of a brushless permanent magnet linear alternator for use with a linear internal combustion engine." PhD diss., West Virginia University Libraries, 1999.

[4] Boldea, I., and S. A. Nasar. "Permanent-magnet linear alternators part 1: Fundamental equations." IEEE Transactions on Aerospace and Electronic Systems 1 (1987): 73-78.

[5] Chen, Yie-Tone, Chun-Lung Chiu, Zong-Hong Tang, You-Len Liang, and Ruey-Hsun Liang. "Optimizing efficiency driver comprising phase-locked loop for the single-phase brushless dc fan motor." IEEE Transactions on Magnetics 48, no. 5 (2012): 1937-1942.

[6] Wang, Jiabin, and David Howe. "A linear permanent magnet generator for a free-piston energy converter." In Electric Machines and Drives, 2005 IEEE International Conference on, pp. 1521-1528. IEEE, 2005.

[7] Li, Qing-feng, Jin Xiao, and Zhen Huang. "Flat-type permanent magnet linear alternator: A suitable device for a free piston linear alternator." Journal of Zhejiang University-SCIENCE A 10, no. 3 (2009): 345-352.

[8] Benvenuto, G., and F. De Monte. "Analysis of free-piston Stirling engine/linear alternator systems. Part I-Theory." Journal of propulsion and power 11, no. 5 (1995): 1036-1046.

[9] Kock, Florian, Johannes Haag, and Horst E. Friedrich. The Free Piston Linear GeneratorDevelopment of an Innovative, Compact, Highly Efficient Range-Extender Module. No. 2013-01-1727. SAE Technical Paper, 2013. 
[10] Mikalsen, R., and A. P. Roskilly. "The design and simulation of a two-stroke free-piston compression ignition engine for electrical power generation." Applied Thermal Engineering 28, no. 5-6 (2008): 589-600.

[11] Djatmiko, Wisnu, and Bambang Sutopo. "Speed control DC motor under varying load using phase-locked loop system." In Proc. of the International Conf. on Electrical, Electronics, Communication, and Information CECI, pp. 7-8. 2001.

[12] Feng, Huihua, Chendong Guo, Chenheng Yuan, Yuyao Guo, Zhengxing Zuo, Anthony Paul Roskilly, and Boru Jia. "Research on combustion process of a free piston diesel linear generator." Applied Energy 161 (2016): 395-403.

[13] Jia, Boru, Zhengxing Zuo, Guohong Tian, Huihua Feng, and A. P. Roskilly. "Development and validation of a free-piston engine generator numerical model." Energy Conversion and Management 91 (2015): 333-341.

[14] Li, Qingfeng, Jin Xiao, and Zhen Huang. "Simulation of a two-stroke free-piston engine for electrical power generation." Energy \& fuels 22, no. 5 (2008): 3443-3449.

[15] Li, Q-F., Jin Xiao, and Zhen Huang. "Parametric study of a free piston linear alternator." International Journal of Automotive Technology 11, no. 1 (2010): 111-117.

[16] Kazemi, Hadi, Yaser P. Fallah, Andrew Nix, and Scott Wayne. "Predictive AECMS by Utilization of Intelligent Transportation Systems for Hybrid Electric Vehicle Powertrain Control." IEEE Transactions on Intelligent Vehicles 2, no. 2 (2017): 75-84.

[17] Kazemi, Hadi, Behnam Khaki, Andrew Nix, Scott Wayne, and Yaser P. Fallah. "Utilizing Situational Awareness for Efficient Control of Powertrain in Parallel Hybrid Electric Vehicles." In Ubiquitous Wireless Broadband (ICUWB), 2015 IEEE International Conference on, pp. 1-5. IEEE, 2015.

[18] Cawthorne, William R., Parviz Famouri, Jingdong Chen, Nigel N. Clark, Thomas I. McDaniel, Richard J. Atkinson, Subhash Nandkumar, Christopher M. Atkinson, and Sorin 
Petreanu. "Development of a linear alternator-engine for hybrid electric vehicle applications." IEEE transactions on vehicular technology 48, no. 6 (1999): 1797-1802.

[19] Tóth-Nagy, Csaba. Linear engine development for series hybrid electric vehicles. West Virginia University, 2004.

[20] Subramanian, Jayaram, Gregory Heiskell, Fereshteh Mahmudzadeh, and Parviz Famouri. "Study of radial and axial magnets for linear alternator-Free piston engine system." In Power Symposium (NAPS), 2017 North American, pp. 1-6. IEEE, 2017.

[21] Johansen, Tor Arne, Olav Egeland, E. Aa Johannessen, and Rolf Kvamsdal. "Free-piston diesel engine timing and control-toward electronic cam-and crankshaft." IEEE Transactions on Control Systems Technology 10, no. 2 (2002): 177-190.

[22] Nandkumar, Subhash. "Two-stroke linear engine." (2000): 1371-1371.

[23] Mao, Jinlong, Zhengxing Zuo, Wen Li, and Huihua Feng. "Multi-dimensional scavenging analysis of a free-piston linear alternator based on numerical simulation." Applied Energy 88, no. 4 (2011): 1140-1152.

[24] Eid, Ahmad M., Ki-Young Suh, Kwang-Ju Choi, Ho-Dong Han, Hyun-Woo Lee, and Mutsuo Nakaoka. "A unique starting scheme of linear-engine tubular PM linear generator system using position feedback controlled PWM inverter." In Power Electronics Specialists Conference, 2006. PESC'06. 37th IEEE, pp. 1-5. IEEE, 2006.

[25] Pavel, Němeček, Šindelka Michal, and Vysoký Ondřej. "Modeling and Control of Linear Combustion Engine." IFAC Proceedings Volumes 37, no. 22 (2004): 305-310.

[26] Zheng, Ping, Chengde Tong, Jingang Bai, Bin Yu, Yi Sui, and Wei Shi. "Electromagnetic design and control strategy of an axially magnetized permanent-magnet linear alternator for free-piston stirling engines." IEEE Transactions on Industry Applications 48, no. 6 (2012): 2230-2239. 
[27] Mikalsen, R., and A. P. Roskilly. "The control of a free-piston engine generator. Part 1: Fundamental analyses." Applied Energy 87, no. 4 (2010): 1273-1280.

[28] Mikalsen, R., and A. P. Roskilly. "The control of a free-piston engine generator. Part 2: Engine dynamics and piston motion control." Applied Energy 87, no. 4 (2010): 1281-1287.

[29] Jia, Boru, Zhengxing Zuo, Huihua Feng, Guohong Tian, and A. P. Roskilly. Development approach of a spark-ignited free-piston engine generator. No. 2014-01-2894. SAE Technical Paper, 2014.

[30] Lane, Neill W., and William T. Beale. "A 5 kW electric free-piston Stirling engine." In Proc, vol. 7, pp. 5-8. 1995.

[31] Kankam, M. David, Jeffrey S. Rauch, and Walter Santiago. "Dynamic analysis of free-piston Stirling engine/linear alternator-load system-Experimentally validated." (1992).

[32] Kankam, M. David, and Jeffrey S. Rauch. "Controllability of free-piston Stirling engine/linear alternator driving a dynamic load." (1994).

[33] Becerra, Roger C., and Mehrdad Ehsani. "High-speed torque control of brushless permanent magnet motors." IEEE Transactions on Industrial Electronics 35, no. 3 (1988): 402-406.

[34] Pillay, Pragasen, and Ramu Krishnan. "Modeling, simulation, and analysis of permanentmagnet motor drives. I. The permanent-magnet synchronous motor drive." IEEE Transactions on industry applications 25, no. 2 (1989): 265-273.

[35] Burger, F., P-A. Besse, and R. S. Popovic. "New single chip Hall sensor for three phases brushless motor control." Sensors and Actuators A: Physical 81, no. 1-3 (2000): 320-323.

[36] Chen, Wei-Chao, and Ying-Yu Tzou. "Current-mode sensorless control of single-phase brushless DC fan motors." In Power Electronics and Drive Systems (PEDS), 2011 IEEE Ninth International Conference on, pp. 659-663. IEEE, 2011.

[37] Wang, Weizi, Zhigan Wu, Wanbing Jin, and Jianping Ying. "Sensorless control technology for single phase BLDCM based on the winding time-sharing method." In Industrial 
Electronics Society, 2005. IECON 2005. 31st Annual Conference of IEEE, pp. 5-pp. IEEE, 2005.

[38] Bianchi, Nicola, Silverio Bolognani, Ji-Hoon Jang, and Seung-Ki Sul. "Comparison of PM motor structures and sensorless control techniques for zero-speed rotor position detection." IEEE transactions on power Electronics 22, no. 6 (2007): 2466-2475.

[39] Ostlund, Stefan, and Michael Brokemper. "Sensorless rotor-position detection from zero to rated speed for an integrated PM synchronous motor drive." IEEE Transactions on Industry Applications 32, no. 5 (1996): 1158-1165.

[40] Shen, J. X., and K. J. Tseng. "Analyses and compensation of rotor position detection error in sensorless PM brushless DC motor drives." IEEE Transactions on Energy Conversion 18, no. 1 (2003): 87-93.

[41] Ha, Jung-Ik, Kozo Ide, Toshihiro Sawa, and Seung-Ki Sul. "Sensorless rotor position estimation of an interior permanent-magnet motor from initial states." IEEE Transactions on Industry Applications 39, no. 3 (2003): 761-767.

[42] Acarnley, Paul P., and John F. Watson. "Review of position-sensorless operation of brushless permanent-magnet machines." IEEE Transactions on Industrial Electronics 53, no. 2 (2006): $352-362$.

[43] Matsui, Nobuyuki. "Sensorless PM brushless DC motor drives." IEEE Transactions on Industrial Electronics 43, no. 2 (1996): 300-308.

[44] Linke, Marco, Ralph Kennel, and Joachim Holtz. "Sensorless position control of permanent magnet synchronous machines without limitation at zero speed." In IECON 02 [Industrial Electronics Society, IEEE 2002 28th Annual Conference of the], vol. 1, pp. 674-679. IEEE, 2002.

[45] Vinatha, U., Swetha Pola, and K. P. Vittal. "Recent developments in control schemes of BLDC motors." In Industrial Technology, 2006. ICIT 2006. IEEE International Conference on, pp. 477-482. IEEE, 2006. 
[46] Hsieh, Guan-Chyun, and James C. Hung. "Phase-locked loop techniques. A survey." IEEE Transactions on industrial electronics 43, no. 6 (1996): 609-615.

[47] Pan, Ching-Tsai, and Emily Fang. "A phase-locked-loop-assisted internal model adjustablespeed controller for BLDC motors." IEEE Transactions on industrial electronics 55, no. 9 (2008): 3415-3425.

[48] Smithgall, David H. "A phase-locked loop motor control system." IEEE Transactions on Industrial Electronics and Control Instrumentation 4 (1975): 487-490.

[49] Abramovitch, Daniel. "Phase-locked loops: A control centric tutorial." In American Control Conference, 2002. Proceedings of the 2002, vol. 1, pp. 1-15. IEEE, 2002.

[50] Prasad, E. S. N., Gopal K. Dubey, and Srinivasa S. Prabhu. "High-performance DC motor drive with phase-locked loop regulation." IEEE transactions on industry applications 1 (1985): 192-201.

[51] Djatmiko, Wisnu, and Bambang Sutopo. "Speed control DC motor under varying load using phase-locked loop system." In Proc. of the International Conf. on Electrical, Electronics, Communication, and Information CECI, pp. 7-8. 2001.

[52] Darzi, Mahdi, Derek Johnson, Chris Ulishney, Mehar Bade, and Nima Zamani. "Baseline Evaluation of Ignition Timing and Compression Ratio Configurations on Efficiency and Combustion Stability of a Small-Bore, Two-Stroke, Natural Gas Engine." In ASME 2017 International Mechanical Engineering Congress and Exposition, pp. V006T08A002V006T08A002. American Society of Mechanical Engineers, 2017. 